
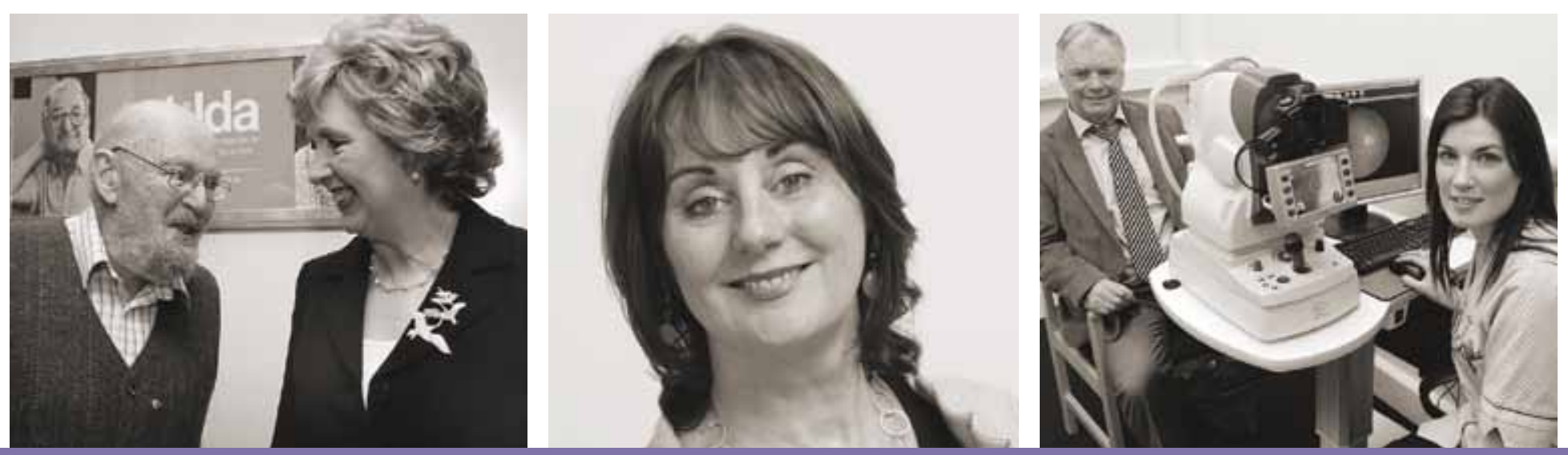

\title{
The Design of the Irish Longitudinal Study on Ageing
}

\section{tida \\ The Irish Longitudinal Study on Ageing}




\section{acknowledgements}

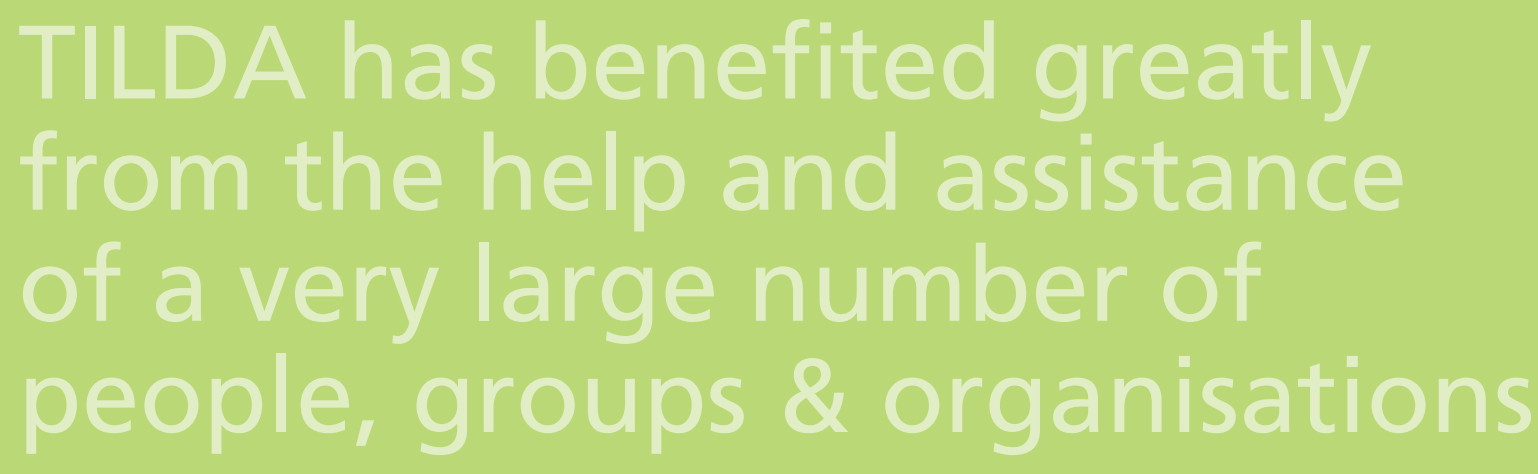

We wish to acknowledge the funding of the project by Irish Life, Atlantic Philanthropies, and the Department of Health \& Children. The support of these early funders allowed this project to be conceived, planned, and implemented.

Thanks are due to members of the TILDA Steering Committee, and most especially the TILDA Executive Committee members, Zhanna O'Clery, Charles Normand, and Virpi Timonen, without whose ongoing help, support and work, TILDA would not be possible. We also wish to thank the TILDA Oversight Board and TILDA Scientific Advisory Board of international experts who continue to provide stewardship of the project, always with a view to serving the needs of the Irish population while helping shepherd Ireland into the international sphere of longitudinal studies on ageing.

We would like to thank the members of our local, national and international research communities who have provided a framework in which TILDA could be designed and implemented. Their advice and suggestions have been invaluable.

We also wish to thank the core TILDA team members who have worked diligently to bring this project to fruition - and to write this report about it. In alphabetical order, they are: Alan Barrett; Hilary Cronin; Yumiko Kamiya; Patricia Kearney; Amilcar Moreira; Jacinta O'Grady; Claire O'Regan; Pauline Walsh; and Mélina Ziegel.

Lastly, we wish to acknowledge the respondents of our study who have given and will be asked to continue to give generously of their time and their thoughts in order for us to understand some very important phenomena in this nation and around the world. 
We hope that you will be as excited as we are by the rich potential of the project and look forward to hearing from you about ideas for collaboration and other activity in this critical area for our future...

Thanks for helping us
make Ireland the best
place in the world to
grow old. 


\section{foreword}

The Irish Longitudinal study on Ageing (TILDA) is a major research undertaking with the potential to radically alter thinking, to enhance policy-making and to promote research and development opportunities in Ireland and elsewhere. In successful models around the world, the results from ongoing longitudinal studies on ageing are considered the primary source of information when governments, decision makers and key community organisations seek to implement change on behalf of the ageing population.

It is my hope that TILDA will become such a pivotal resource for Ireland. As such, this document is intended to inform the research community about the TILDA design and its content, focusing specifically on the justification of its key investigation areas and methodology choices and how these compare to international standards.

It has proved a rather complicated task to decide what to include here and how best to present our project. TILDA collects economic, health and social data across a variety of research questions using several modalities. For example, health information, both subjective and objective, is collected and recorded in the Computer-Aided Personal Interview (CAPI) and Self-Completion Questionnaires (SCQ) and in the Health Assessment (HA). The benefit of this methodology is that researchers can triangulate phenomena about an area of inquiry using data collected in different ways. This report is organised by modality (i.e. type of questionnaire or assessment) rather than by content area. This will allow researchers to understand the data respondents provide in the sequence in which it is collected.

The TILDA team and I hope that this document helps convey the considerations undertaken in implementing the study and the richness of the data that will become available when fieldwork ends. We hope that you will be as excited as we are by the rich potential of the project and look forward to hearing from you about ideas for collaboration and other activity in this critical area for our future.

Thanks for helping us make Ireland the best place in the world to grow old.

Rose Anne Kenny

TILDA Principal Investigator 


\section{table of contents}

1. Introduction, Objectives and Sample Design.....................................................

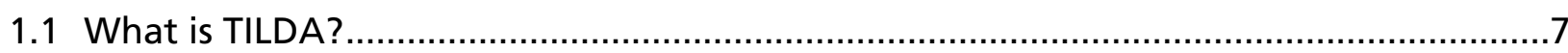

1.2 The Need for a Longitudinal Study on Ageing in Ireland ...........................................

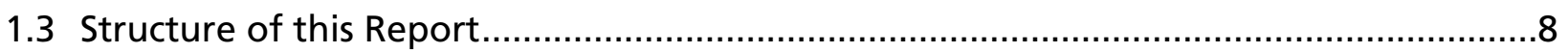

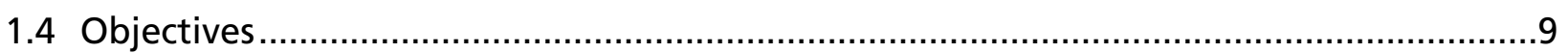

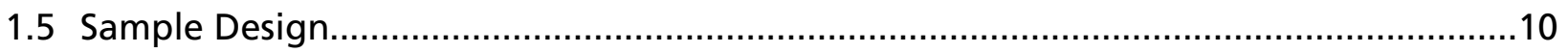

2. CAPI Questionnaire: Coverscreen and Demographics............................................... 13

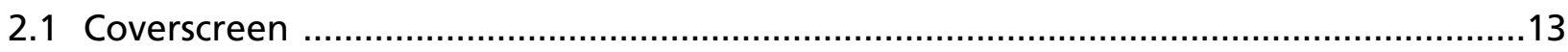

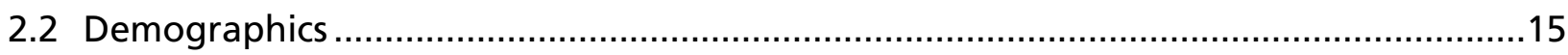

3. CAPI Questionnaire: Social Circumstances ...................................................................17

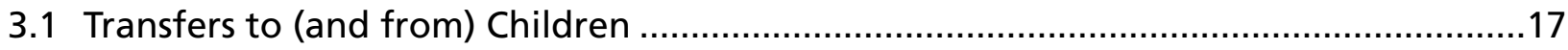

3.2 (Instrumental) Activities of Daily Living and Helpers .................................................20

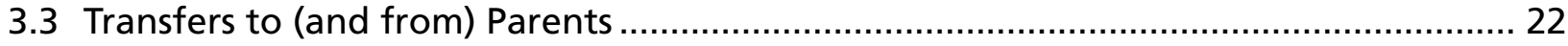

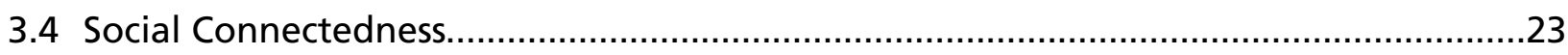

4. CAPI Questionnaire: Health and Healthcare ........................................................... 25

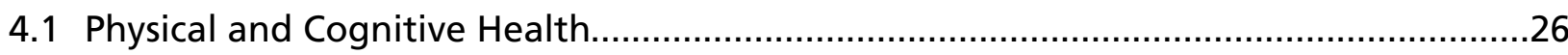

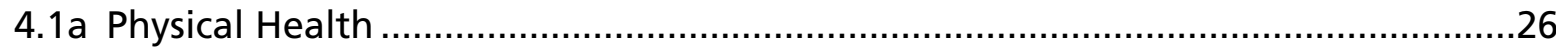

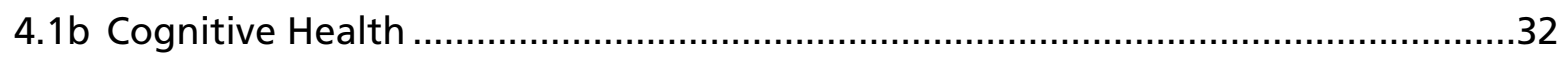

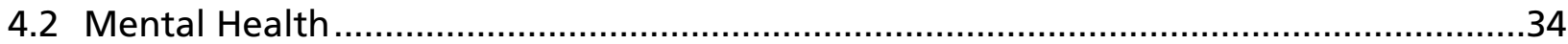

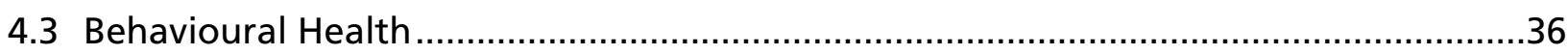

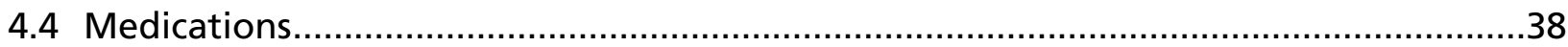

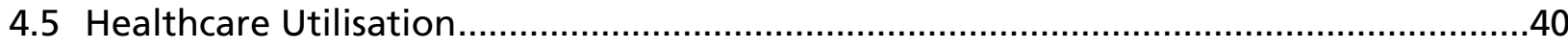

5. CAPI Questionnaire: Employment and Lifelong Learning ........................................ 43

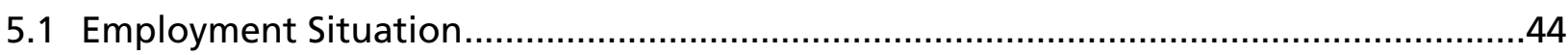

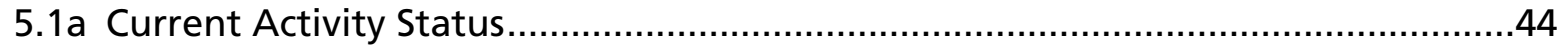

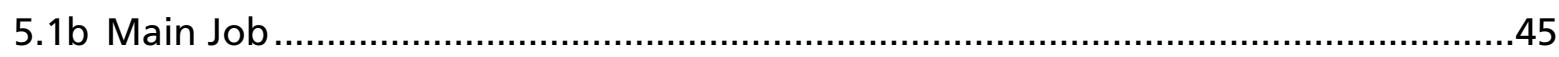

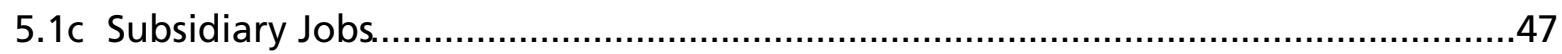

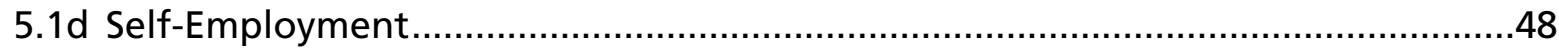

5.1e Unemployment and Permanent Sickness/Disability ........................................49

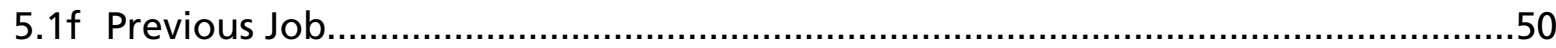

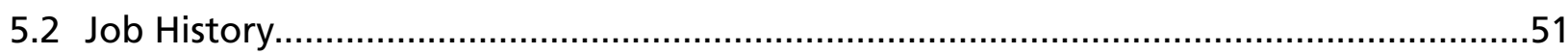

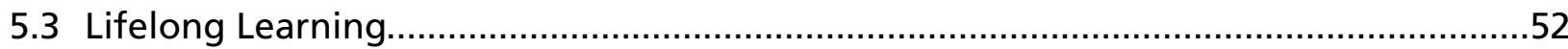


6. CAPI Questionnaire: Planning for Retirement and Expectations ..................................53

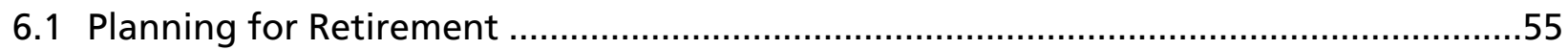

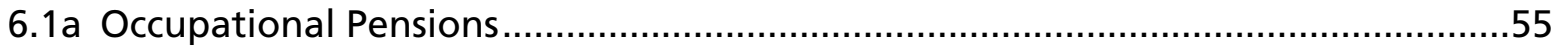

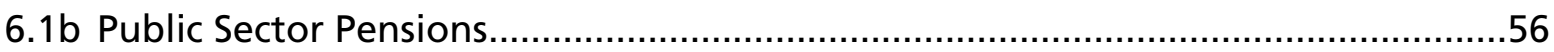

6.1c Personal Retirement Savings Accounts.............................................................57

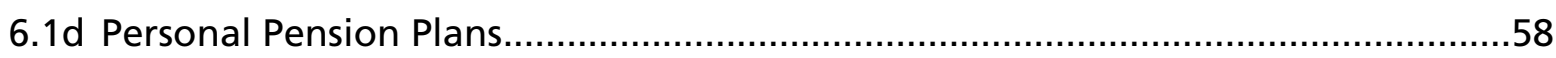

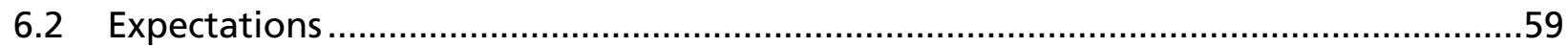

7. CAPI Questionnaire: Sources of Income, Assets and Transport.....................................61

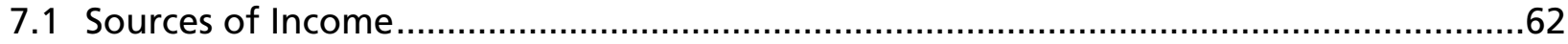

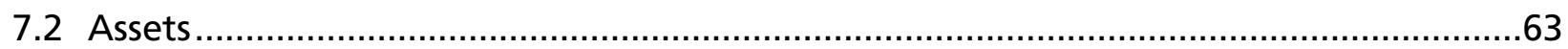

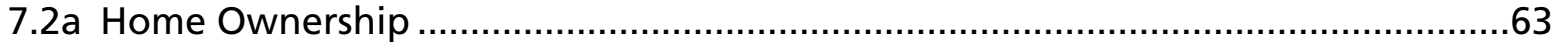

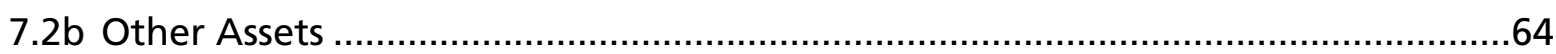

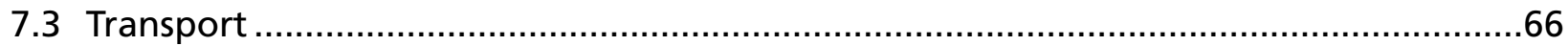

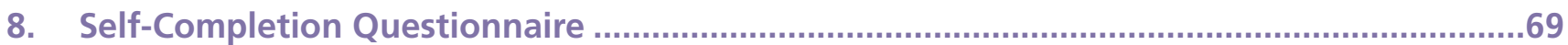

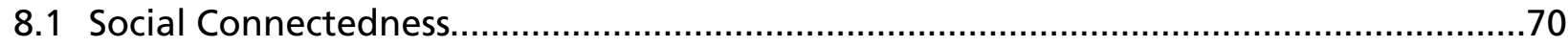

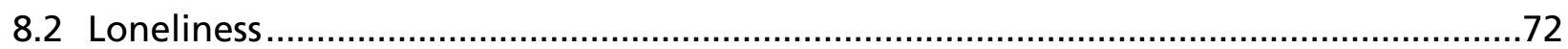

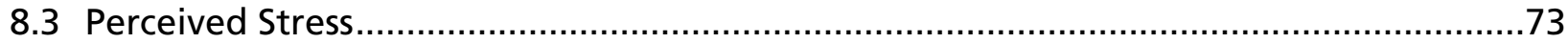

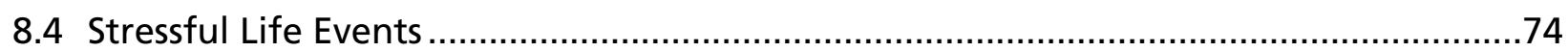

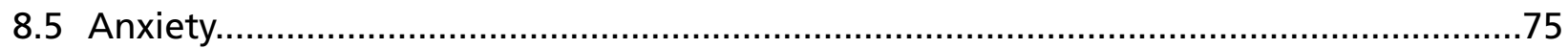

8.6 Worry

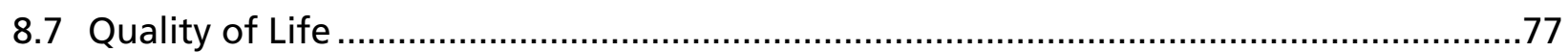

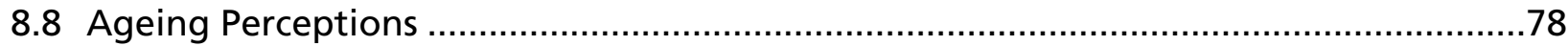

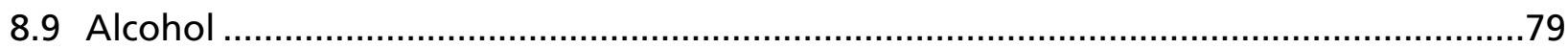

9. Health Assessment: Tests and Procedures ......................................................... 81

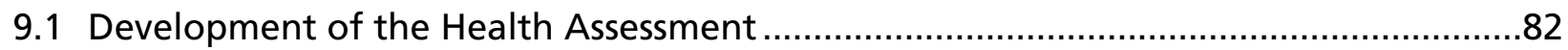

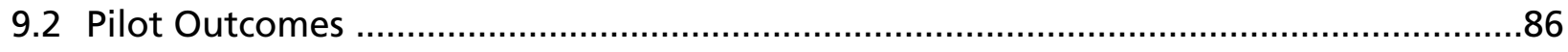

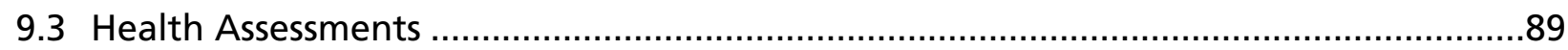

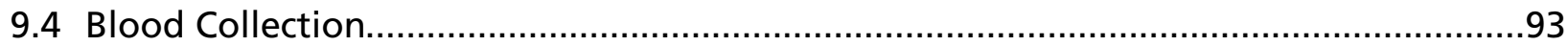

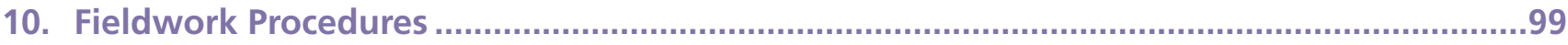

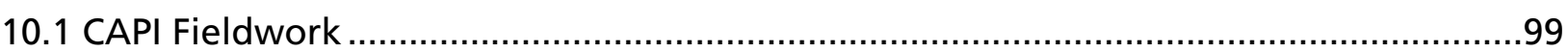

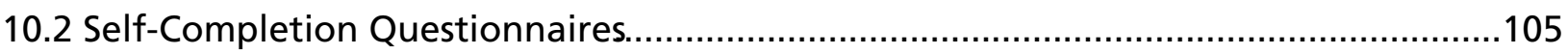

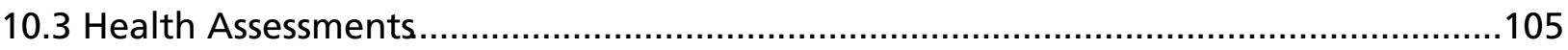

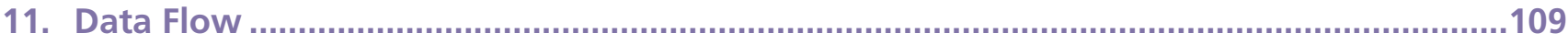

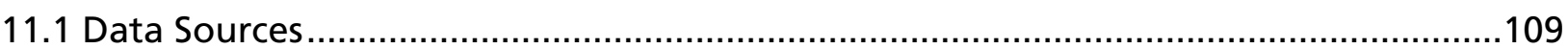

11.2 Comments on Limitations of this Report ...............................................................110

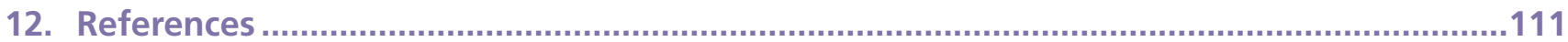

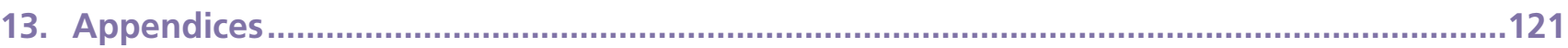




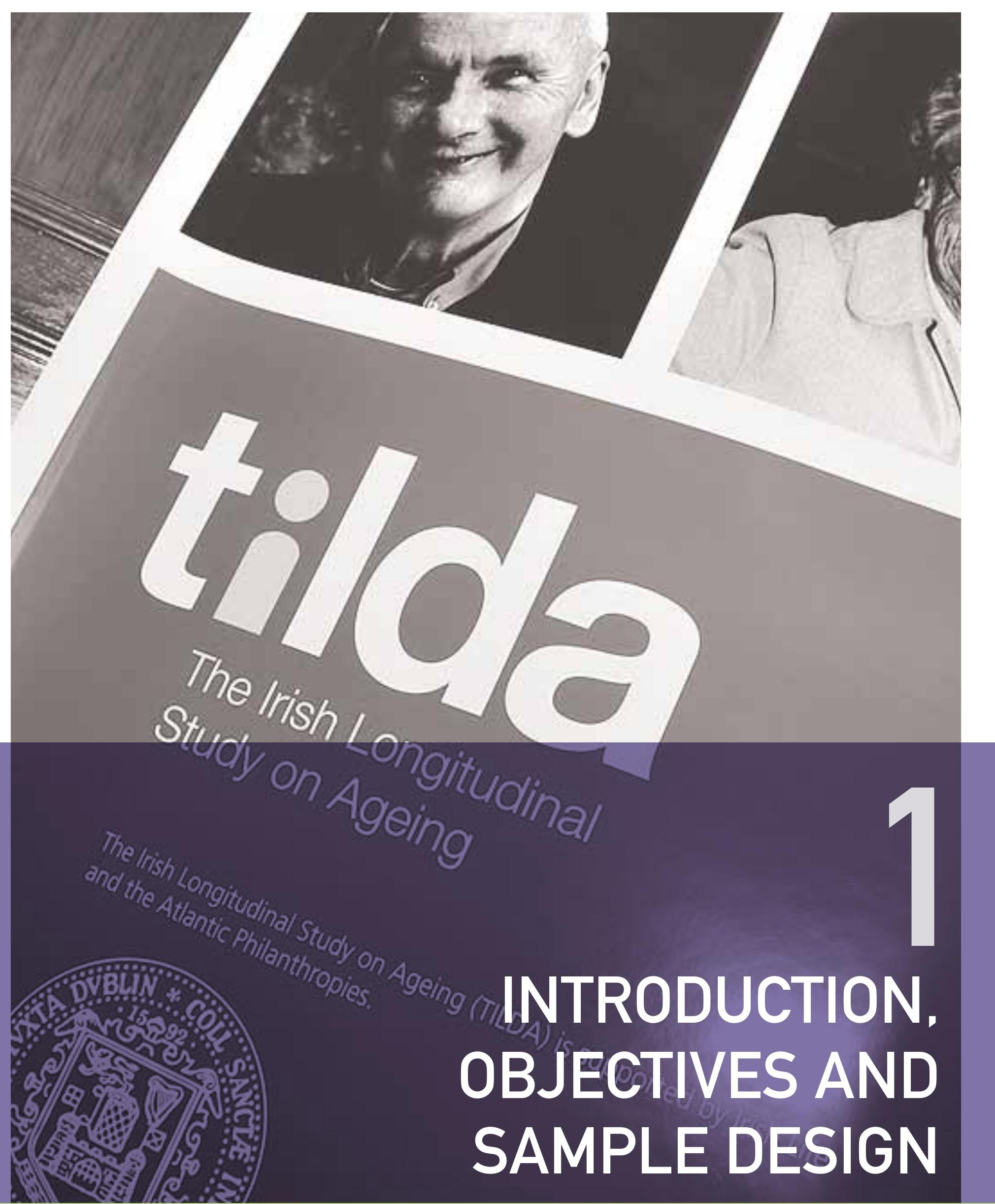

<18......

... Niai

......... 


\section{1 introduction, objectives and sample design}

\subsection{What is TILDA?}

The Irish Longitudinal study on Ageing (TILDA) is a major inter-institutional initiative led by Trinity College Dublin which aims to produce a massive improvement in the quantity and quality of data, research and information relating to older people and ageing in Ireland. Eligible respondents for this study include individuals aged 50 and over and their spouses or partners of any age. The study will involve interviews on a two yearly basis with a sample cohort of 8,000+ people aged 50 and over (or their spouses/partners) and resident in Ireland, collecting detailed information on all aspects of their lives, including the economic (pensions, employment, living standards), health (physical, mental, service needs and usage) and social aspects (contact with friends and kin, formal and informal care, social participation). Both survey interviews and physical and biological measurements are utilised. The study is closely harmonised with leading international research so as to ensure adoption of best practice and comparability of results.

\subsection{The Need for a Longitudinal Study on Ageing in Ireland}

Population ageing will have a profound and pervasive effect on our global society in the future. In 1993, one out of every ten persons in the world was aged 60 or over but projections indicate that by 2050 this ratio will have increased to one in five (United Nations, 2002). This ageing transformation will pose a formidable challenge: how to sustain an adequate standard of living and quality of life for older people, and to provide appropriate services and facilities such as healthcare and social care, while ensuring the effectiveness and financial sustainability of our systems and institutions. Countries throughout the world are realising that creative policy initiatives will be needed to address this challenge. New policies will, in turn, require a greatly enhanced research base if they are to be well designed and effective.

In Ireland, the proportion of the population aged 65 and over has remained steady at about $11 \%$ for the past forty years. However, it is projected that this proportion will rise to $14 \%$ by 2021 and to $19 \%$ by 2031 . The greatest increase will be in the oldest old, the over 80 's, whose share of the population will more than treble by 2036 (Central Statistics Office, 2004).

The growing importance of this trend has been recognized by Irish policy makers. For example, the Towards 2016 Partnership Agreement (Department of the Taoiseach, 2006), gives a prominent role to improving all aspects of the lives of older people, including pensions and income support measures, long-term care services, housing and accommodation, mobility issues, health and healthcare and education and employment opportunities. The Irish National 
Social Inclusion Strategy includes older people as one of its key target groups. A new Minister of State with responsibility for older people has been appointed and the Programme for Government 2007 proposes the creation of a National Strategy for Older People. This Strategy will incorporate the development of operational plans by Government Departments clearly setting out objectives relating to older people.

TILDA, with its unique multi-domain, longitudinal design, will provide the essential research base for developing these policies. It will:

- $\quad$ give comprehensive baseline data on older people in Ireland, leading to improvements in policy and planning;

- $\quad$ provide new insights into the causal processes underlying the ageing transformation;

- $\quad$ add to the prominence of ageing as an issue of public interest and allow the voice of older people to be heard more clearly, by effectively disseminating results to various audiences;

- lead to further extensive analysis by academic researchers both in Ireland and abroad, helping to create an enhanced infrastructure for ageing research in Ireland and to attract international scholars and funding, by making its anonymised dataset openly available.

In this way, TILDA will contribute to the creation of Ireland as a "knowledge society".

\subsection{Structure of this Report}

\section{Chapter one sets out the major objectives of TILDA and the sample design.}

Chapters two through seven describe the major content areas of the Computer-Aided Personal Interview (CAPI) questionnaire, organised by the modules in the CAPI. These six chapters all have the same organisational structure, as follows:

- Rationale for inclusion of this module

- Measures

- Logic for choice

- Comparability with other (longitudinal) studies

The 'Rationale for inclusion of this module' explains the significance of a particular topic or research area, paying specific attention to its relevance for the Irish population. In general, this section identifies the type of research question that can be investigated using the data captured in this module. The 'Measures' section describes the specific content of the individual (sets of) questions covered by each module. The 'Logic for choice' relates the questions included in the CAPI to the research questions that can be answered by them, in part by addressing current thinking on Irish and worldwide trends. Lastly, 'Comparability with other (longitudinal) studies' describes the extent to which the measures selected are comparable 
with other major studies on ageing. In all cases, the TILDA measures are compared against three studies: The English Longitudinal Study of Ageing (ELSA); the Survey of Health, Ageing and Retirement in Europe (SHARE) which is pan-European, and the Health and Retirement Survey (HRS) conducted in the United States. The comparison will appear in a table where the TILDA module is named and ticks appear in the column for each of other studies with the same (sets of) questions. In addition, where applicable, comparisons with studies besides these three are also made, in the text.

Chapter eight describes the content of the Self-Completion Questionnaire, which was designed to allow TILDA to explore certain areas that were considered particularly sensitive for respondents to answer directly to an interviewer. Chapter nine describes the design and methodology of the health assessment component of the study, conducted both in dedicated TILDA health assessment centres and, alternatively, in respondents' homes. As most of the measures in the health assessment are novel, this chapter is more theoretical in its approach as TILDA had fewer or no models from which to learn best practices and designed its kit ab initio. In this instance, TILDA hopes to set the standard for health assessments in longitudinal studies going forward. Chapter ten describes fieldwork procedures, including the outcomes of the first two pilots and how those findings affected the subsequent steps. Chapter 11 briefly describes the TILDA data flow plan. References and appendices follow.

\subsection{Objectives}

The principal objectives of the study are as follows:

1. To provide an integrated and representative database covering all the key domains of older adults' lives including health, income, living conditions, social contact, accommodation, environment and family circumstances. A key feature of the study is its multi-disciplinary nature. By establishing and nurturing real collaboration between experts in a range of disciplines, it will be possible for the first time to study in depth how these different domains relate to each other, and to locate the points at which policy interventions are most appropriate. This approach is essential for understanding the complex web of causation involving health, income and social circumstances.

2. To collect longitudinal data i.e. information on the same individuals at different points in time. This permits researchers to describe and understand the fundamental causal processes involved, which would not be possible with cross-sectional (point-in-time) data.

3. To give older people a voice within the national conversation by documenting and publicising their concerns, experiences and expectations. The study will demonstrate the substantial contribution that older people make to society and how it can be enhanced. Too often, older people are seen as a group which is a burden rather than active contributors to society. TILDA will establish the potential to radically alter this stereotype.

4. To provide comprehensive base-line data on older people in Ireland. The large, high quality sample will permit substantial disaggregation, permitting researchers to study relatively 
small sub-groups in the population. The database will also provide norms in the sense of establishing what is typical or usual, with which specific sub-groups (e.g. individuals suffering from a particular disease or disability) can be compared.

5. To link closely with, and learn from, the best international studies in the area. This is vital for ensuring that the methods used conform to best international practice. Ireland has a number of unique features with respect to ageing which means that strategies or policy solutions adopted abroad will not always work in the Irish context. It is, therefore, essential to develop an understanding of what is, and what is not, truly comparable between Ireland and other countries.

6. To collaborate closely with other cutting-edge research initiatives in Ireland. Contact and collaboration has already been established with researchers working in a range of areas at international level including technological research, molecular medicine, neuroscience, poverty measurement and macular degeneration.

7. To build an understanding that ageing affects more than the old. Population ageing will affect overall demand and consumption patterns, the public finances and the labour market. For instance, the caring occupations are among the occupational groups predicted to grow most rapidly over the next 10 years.

8. To build capacity in ageing research in Ireland. The existence of a rich data source will attract top quality researchers from abroad and encourage the best Irish and international students to consider research in ageing as an important and attractive field. Also, a number of multinational firms are prioritising such research as key target areas for future investment. TILDA will position Ireland as a contender for these projects by providing base-line data and offering the possibility of linked studies.

\subsection{Sample Design}

\subsubsection{Target Population}

The target population for the TILDA survey is the population of persons aged 50 or over living in residential addresses in the Republic of Ireland, and their spouses or partners of any age. A probability sample of this population gives each member of the population a known, non-zero probability of being selected.

\subsubsection{Possible Sampling Frames}

An appropriate sampling frame is absolutely essential to any survey of the population. Without a high quality frame, the results of the study risk being undermined to the point of being useless. Kish (1965) defines an ideal frame as one on which "every element in the population appears once, only once and nothing else appears on the list". 
Two possible sampling frames were considered for selecting the TILDA sample:

(a) the RANSAM system, based on the Geodirectory, which has been developed by the Economic and Social Research Institute (ESRI) of Ireland (Whelan, 1979).

(b) the Register of names and addresses of persons with Personal Public Service (PPS) Numbers maintained by the Client Identity Services Division of the Department of Social and Family Affairs. The PPS Register is used extensively in administering the national social welfare system (pensions, unemployment payments, child benefit etc.).

The former has been used for a variety of surveys and its properties are well known. The PPS Register has not been used for sampling purposes before.

A detailed analysis of each sampling frame is in Appendix 1.

Having systematically evaluated the two sources, it was found that the numbers on the PPS Register substantially exceeded the corresponding numbers recorded in the Census of population, and that this over-representation was particularly concentrated among men and older age groups. Since this would introduce bias into the sample, it was decided to proceed with the RANSAM system as a basis for generating the sample for the TILDA survey.

\subsubsection{Sample Design}

The TILDA pilots were conducted using samples based on the RANSAM system. The pilots were able to provide evidence of the likely levels of non-eligibility (vacant dwellings, institutions wrongly included etc.) and response rates (refusals, non-contacts, etc.). These samples also conformed to the expected pattern of approximately half of the addresses being ineligible because they did not contain a person aged 50 or over. Statistical and resource factors determined the minimum required sample size at 8,000 persons to provide national representation for the TILDA baseline. The target response rate is 60 per cent of eligible households.

\section{This information allowed us to specify a two-stage sample design as follows:}

\section{Stage 1}

RANSAM groups the residential addresses in the country into 3,155 first stage units or clusters. These clusters are townlands or aggregations of townlands and range in size from 500 to 1180 addresses. It was decided to select 640 of these clusters, with implicit proportionate stratification of clusters by socio-economic group (3 categories) and geography. Characteristics of the clusters can be inferred from the District Electoral Divisions of which they are a part, on the basis of the Small Area Population Statistics compiled by the Central Statistics Office.

Stratification was achieved by pre-sorting all addresses in the country by socio-economic group (three equal groups on the basis of percentage of the population in the professional/ managerial category) and within socio-economic group by RANSAM's geographical "snake" 

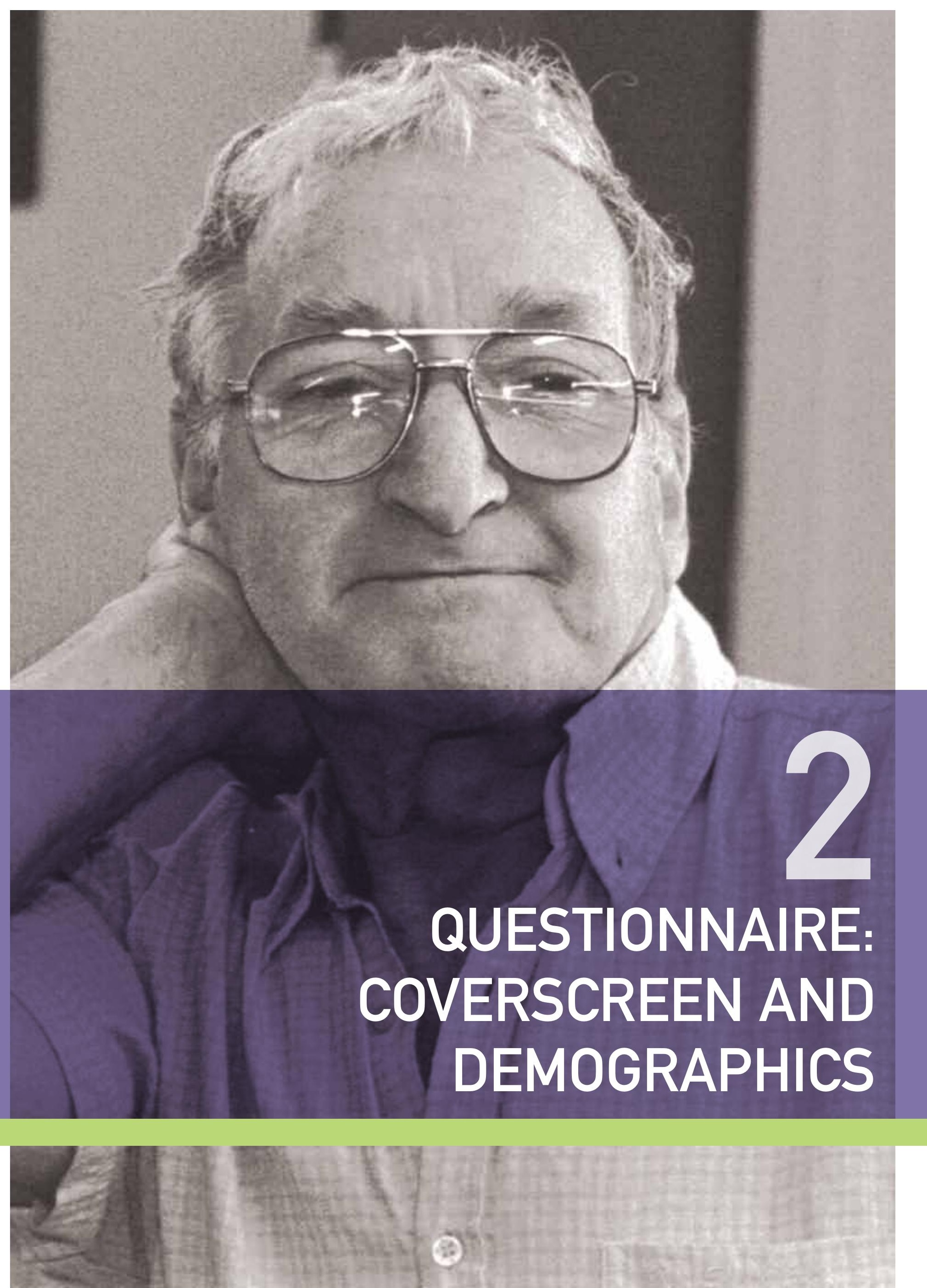
pattern which orders clusters within county based on a north/south pattern which preserves contiguity. Clusters were selected randomly with a probability of selection proportional to the estimated number of persons aged 50 or over in each cluster.

\section{Stage 2}

This stage involved the selection of a probability sample of 50 addresses within each cluster (10 to be held in reserve). The combination of selection probabilities used at the two stages produces an equal probability ("epsem") sample of addresses. All persons aged 50 or over in the selected households (and their spouses or partners of any age) are asked to participate.

The addresses are partitioned into two groups: an initial sample list of 25,600 addresses (40 randomly selected from each of the 640 clusters) for immediate issue to the field force and 6,400 addresses (10 randomly selected from each of the 640 clusters) for retention as a reserve list. The reserve list will only be utilised later in the fieldwork process if it appears unlikely that the target sample size will be achieved. If the target sample of 8,000 interviews can be obtained from the initial list, the information from the pilot work suggests that the response rate will be just in excess of 60 per cent.

As described, the sample design incorporates stratification, clustering and multi-stage selection. The design results in an equal probability sample of both households containing members of the target population and of persons in the target group. This means that the resulting sample is "epsem" and self-weighting, except for biases caused by non-random variations in response rates. Such biases will be dealt with at analysis stage by means of calibration weights.

\subsubsection{Sampling Errors}

In terms of the efficiency of the design, stratification and clustering operate in opposite directions. The proportionate stratification used at stage 1 will reduce sampling errors below those of a simple random sample. Clustering, on the other hand, will increase sampling error since members of clusters are likely to resemble each other leading to positive intra-cluster correlation co-efficients. When fieldwork is completed, "design effect" calculations will be carried out to determine how this balance has worked out and to provide researchers with guidance regarding the ultimate level of sampling error and advice regarding significance testing. 


\section{2 questionnaire: coverscreen
and demographics}

\subsection{Coverscreen}

The TILDA questionnaire, like many studies, begins with a coverscreen section that confirms eligibility of a given respondent and captures some basic information about other members of the household. The TILDA coverscreen has two parts: the household coverscreen and the individual coverscreen. These are described in more detail below:

\subsubsection{Rationale for inclusion of this module}

The TILDA sample consists of people living in residential households who are 50 years of age or older at the time of participation, and their spouse/partner of any age.

The Computer-Aided Personal Interview (CAPI) questionnaire includes two coverscreens and the main questionnaire. The interview starts with the household coverscreen, which is completed by the first informant interviewed and allows him/her to share information about all household members. The second coverscreen relates to each individual participating in the study.

The household coverscreen aims to collect a complete list of all people living in the household and to determine which individuals in the household are eligible for participation in the main questionnaire. The questions that are captured in the household coverscreen include the age and relationship to the respondent of each household member living with the respondent. They also identify whether individuals are part of a couple (married or living as married with their spouse/partner) or not. This information is essential to determine which household members are eligible to participate in the study. Information from the household coverscreen is fed forward to subsequent respondents in the household while the second coverscreen is asked of all eligible persons in respect of their own personal characteristics. The second coverscreen also contains the statement of confidentiality confirming that each eligible respondent has signed the consent forms and authorised a fieldwork agency to return for future contact, as this is a longitudinal study where future data collection is planned.

In order to minimise the duration of the interview in multi-person households, some components of the questionnaire are allocated to one household member only. In households with only one respondent, all questions (modules) of the main instrument are presented to the respondent. 
In order to establish which set(s) of questions is/are presented to a respondent in a multiperson household, CAPI asks who is most knowledgeable in relation to family finances; this person then becomes the 'financial respondent'. The 'financial respondent' answers the Sources of Income, House Ownership and Other Assets modules. The 'family respondent' is the person who is most knowledgeable about family issues and answers the questions in the Transfers to Children section.

In summary, in one-person households, the respondent is always the financial and family respondent. In multi-person households, an individual respondent may act as the financial or family respondent, as both, or neither of these. For some households, there may be more than one financial and family respondent, one for each couple unit in the household. Respondents living without a partner in multi-person households are always both financial and family respondents.

After completing the coverscreens, eligible persons proceed to the main questionnaire, which consists of 24 modules in total.

The TILDA questionnaire was developed to meet the following criteria:

1) multidisciplinarity;

2) longitudinality; and

3) comparability with other major studies of ageing and with national statistical sources.

As in all longitudinal studies, many of the design decisions involved difficult trade-offs between interview length and the degree of detail to be collected.

\subsubsection{Measures}

The household coverscreen asks basic descriptive questions about each member in the eligible household, including their sex, age and relationship to each other. The respondent coverscreen collects some more detail in respect of each eligible respondent. In cases where both members of a couple indicate their willingness to participate, the first informant identifies the financial and family respondent here.

\subsubsection{Logic for choice}

The questions asked in the coverscreen include basic information on household members and questions related to consent and the identification, where relevant, of financial and family respondents

\subsubsection{Comparability with other (longitudinal) studies}

All household members aged 50 and over, plus their spouses or partners of any age are eligible to participate in TILDA, ELSA, and SHARE. However, in HRS, if a household contains more than one unrelated age-eligible person (or couple), one of these persons (couples) is randomly selected for interview. 


\begin{tabular}{|l|c|c|c|}
\hline TILDA & ELSA & SHARE & HRS \\
\hline Household Roster & $\checkmark$ & $\checkmark$ & $\checkmark$ \\
\hline Selecting Financial / Family Respondent & $\checkmark$ & $\checkmark$ & $\checkmark$ \\
\hline
\end{tabular}

TILDA, ELSA, SHARE and HRS compile a household roster and identify the person who is most knowledgeable on family and/or financial matters to respond to more detailed questions in these domains. All of these studies collect comparable basic information on the household members.

\subsection{Demographics}

\subsubsection{Rationale}

The objective of this section is to obtain core demographic data and information on the context of the respondent's life, including marital status and marital history, education, childhood information and migration.

The rationale for collecting this information lies in the large literature emphasising the influence of marital status, childhood and education on the incidence of health events, health status and quality of life during adult years. For example, married people have significantly better health and lower mortality than their single counterparts (Smith and Zick, 1994). These results are particularly strong for men (Lillard and Panis, 1996; Hu and Goldman, 1990). Widowed, divorced and never married individuals have higher mortality than married people (Korenman and Goldman, 1993; Goldman et al., 1995; Mineau et al., 2002). Education is widely acknowledged to be one of the most important socio-economic determinants of health and mortality. There is considerable evidence that low educational attainment is strongly correlated with disease, health behaviour risks and mortality (Elo and Preston, 1996; Pappas et al., 1993). With respect to childhood, a strong relationship between parental income and childhood health has been documented. Adult socio-economic characteristics (education, employment, income) and health outcomes are also correlated (Preston, et al., 1998; Almond et al, 2007). For instance, Case, Fertig and Paxson (2005) have reported on the influence of childhood health and socio-economic status (SES) on adult health and SES using the British birth cohort study.

With respect to questions on migration, Ireland has a long history of significant emigration. Between 1871 and 1961, the average annual net emigration from Ireland consistently exceeded the natural increase in the Irish population, which shrank from about 4.4 million in 1861 to 2.8 million in 1961. An estimated 3 million Irish citizens currently live abroad, of which 1.2 million were born in Ireland. The majority live in the US and the UK. However, the booming economy of the late 1990's led to an increase in net immigration, largely because of the return of Irish workers and their families and new immigrants coming (predominantly but not exclusively) from Central and Eastern European countries. Consequently, the population of Ireland has increased considerably in recent years, in contrast to the trend in many other European countries. The TILDA questionnaire is designed to capture the effects of migration history on the lives of older people in Ireland. 


\subsubsection{Measures}

The demographic section asks respondents questions about their childhood and the characteristics of their family of origin. Respondents are asked about the highest level of education achieved by themselves and both their mother and father. They are asked where their parents grew up as well as where they themselves grew up, specifically whether the area they lived in during their early years was rural or not. Respondents are asked to self-report their health and family's economic status during their childhood. They also indicate whether each of their parents worked outside the home when they were children and if so, what type of job that parent had. Lastly, respondents answer a set of questions about their nationality and about any countries other than Ireland in which they have spent a significant amount of time (i.e. more than six months).

\subsubsection{Logic for choice}

The demographics section is standard to collect basic information about respondents, as described above.

\subsubsection{Comparability with other (longitudinal) studies}

Similar questions on marital status, marriage history, childhood and education are included in ELSA, SHARE and HRS. Migration history questions are included only in HRS.

\begin{tabular}{|l|c|c|c|}
\hline TILDA & ELSA & SHARE & HRS \\
\hline Marital Status and Marriage History & $\checkmark$ & $\checkmark$ & $\checkmark$ \\
\hline Education & $\checkmark$ & $\checkmark$ & $\checkmark$ \\
\hline Childhood Health & $\checkmark$ & $\checkmark$ & $\checkmark$ \\
\hline Migration History & & & $\checkmark$ \\
\hline
\end{tabular}



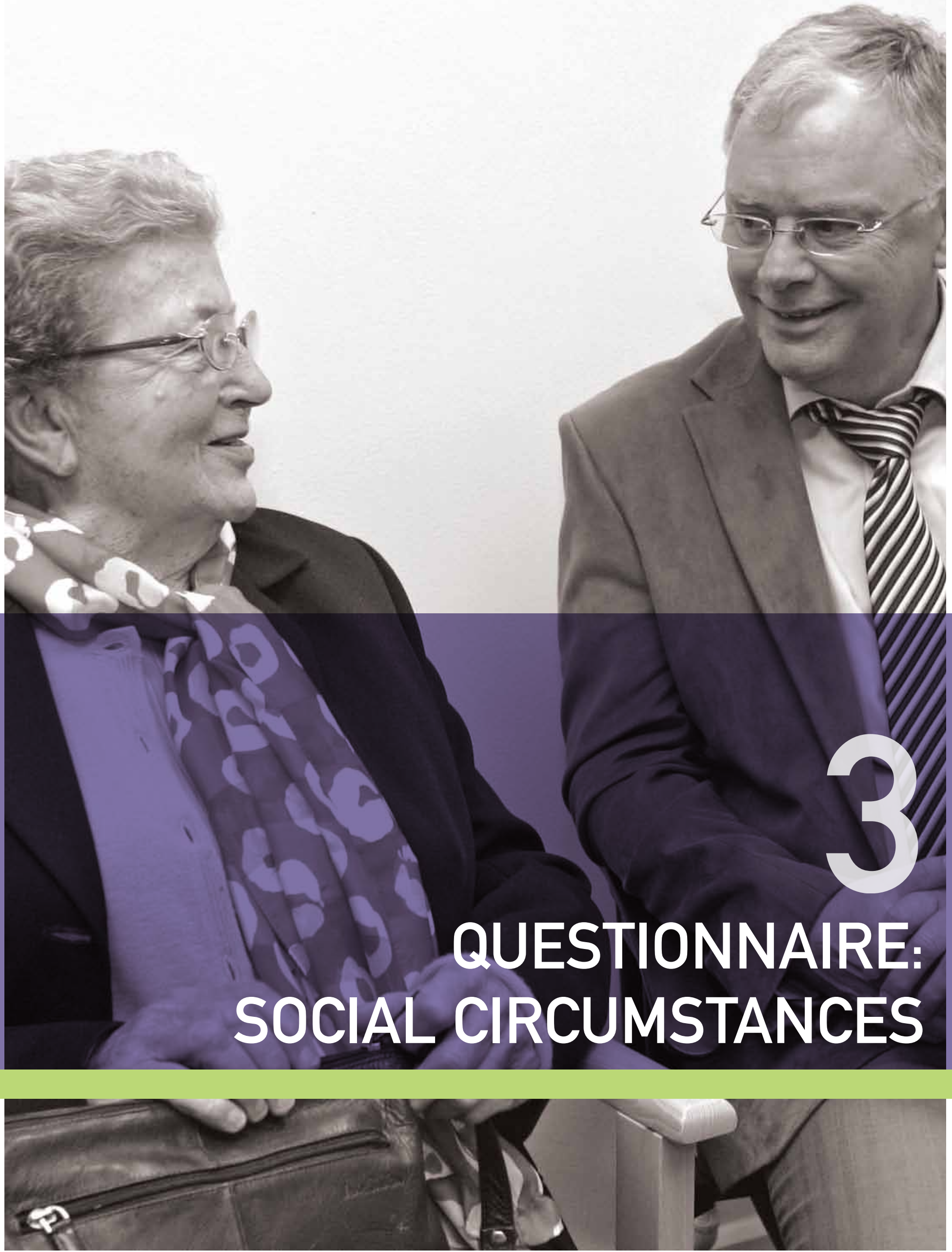


\section{3 questionnaire: social circumstances}

\section{Introduction}

This chapter will provide a comprehensive portrait of older peoples' social circumstances. In order to create an age-friendly society, and to improve service delivery to older people, policy makers need to know who these people are, and understand their individual needs and perspectives. TILDA will provide the data needed to examine the complex relationship between socio-economic status and health, to understand family and household structures and intergenerational transfers, and to explore the dynamic relationship between health, social integration and economic position.

The social modules included in the CAPI questionnaire are:

1. Transfers to (and from) Children

2. (Instrumental) Activities of Daily Living and Helpers

3. Transfer to (and from) Parents

4. Social Connectedness

\subsection{Transfers to (and from) Children}

\subsubsection{Rationale for inclusion of this module}

The rationale for this section also applies to the Transfers to (and from) Parents section and the Instrumental Activities of Daily Living \& Helpers sections that follow.

In England and in the United States, around one-third of women aged 55-69 have reported providing help to ascending and descending generations simultaneously (Grundy and Henretta, 2006). There is no similar data available for Ireland. It is of great interest from the point of view of understanding older people's lives and for policy and service planning purposes, to determine whether the corresponding group in Ireland exhibits the pattern of a "sandwich generation" with substantial caring responsibilities towards both ascending and descending generations.

In order to capture the complexity of exchanges (support given and received) between different generations, we define intergenerational transfers as "a generic term used to describe the redistribution of resources within an extended family structure, incorporating 
both intrahousehold and interhousehold exchanges" (Soldo and Hill, 1993). Nevertheless, the focus on family does not diminish the importance of and the need to collect information on support received from formal sources (such as the state, private service providers, and nonprofit organisations) and from informal sources other than the family (friends and neighbours). Measuring both informal and formal support is important because the provision of formal health and social care services interacts with and affects the type and intensity of the support given by the family to older persons.

Three major 'currencies' can be transferred between the different generations of a family, namely space, money and time. The first one is usually measured by co-residence. The second refers to financial assistance (e.g. financial assistance for education, purchase of a home) as well as goods (e.g. food, clothing). The third category of transfers, time, includes the provision of care to children (or parents in the later section), assistance with household duties, and emotional support.

As shown in Table 3.1 below, transfers in the intergenerational support system can go from left to right or from right to left. This table illustrates the flow of transfers from the persons in the 'middle' generation to potential recipient generations, i.e. their parents and their children. However, transfers can be more complicated. For example, a parent might provide housing and financial help to a recently divorced adult child. The child in turn may provide personal care assistance for one of the parents. In this case there are three currencies involved simultaneously (space, time and money). These transfers can vary in intensity (magnitude) and depend on the needs of the recipients as well as the constraints of the donors.

Table 3.1 Intergenerational Transfer Support System

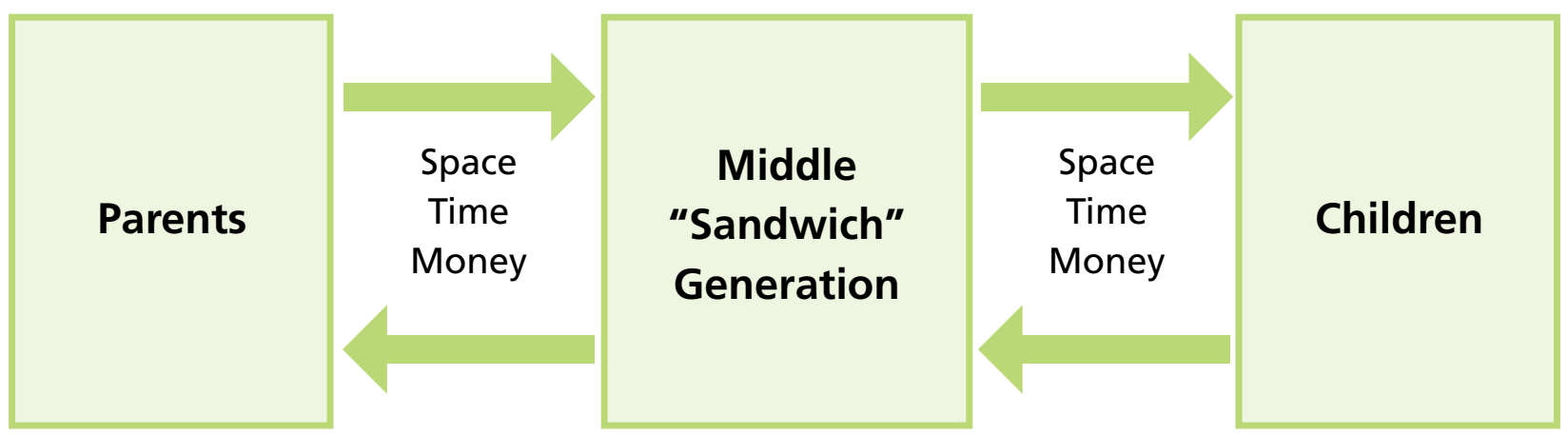

Given the complexity of transfers described above, the best way to capture all the dimensions of transfers is to: first, compile a roster of family members (within and outside the household); second, utilise the correct sequence of questions regarding the intensity (magnitude), direction (from whom to whom), purpose (physical care, child care, etc.), timing (current or past, persistence of exchange over time), form, and impact (needs of recipient, resources of provider) of transfers (Hermadin, 2000). 
The strengths of TILDA for analyses of intergenerational transfers are that it:

1) provides information on the multiple currencies of intergenerational exchange (financial, time, and co-residence); i.e. TILDA allows researchers to consider transfers in all three currencies made to linear kin;

2) provides information on simultaneous flows of help given by the respondents to kin in ascending and descending generations; and,

3) provides data on the allocation of transfers between and among multiple benefactors in Generation 1 and Generation 3.

This data is laborious to collect and represents a major TILDA investment that should have an especially high payoff in understanding the intergenerational relationships and transfers among older Irish persons, an area on which very little data exists at the moment.

\subsubsection{Measures}

Although we usually refer to this module as "Transfers to Children", TILDA in fact collects information on transfers both to and from children so the titles "Transfers to Children" and "Transfers to (and from) Children" may be used interchangeably. The Transfers to Children section includes questions about respondents' children living outside the home, including their geographic proximity. (As described earlier, information on resident children is captured in the Household Coverscreen at the start of the first informant's interview). In this section, TILDA collects information about transfers of money and time to and from children. Respondents quantify the specific amounts of euros of financial help transferred as well as the number of hours per month that help was provided with specific types of tasks. This data is important for understanding how the intermediate generation allocates resources to competing generations within the family. Over time, TILDA will enable researchers to study reciprocal flows of support between parents and children, and the effect of these transfers on labour supply.

\subsubsection{Logic for choice}

The TILDA design is modelled on the HRS questionnaire. It enables researchers to track the main transfers across generations. However, because of the trade-off between the content and length of the questionnaire, we do not map in full detail the exchanges that take place with specific children in a family.

\subsubsection{Comparability with other (longitudinal) studies}

The measurement of transfers to and from children in TILDA is designed to be comparable with SHARE and HRS who investigate intergenerational transfers in all possible directions. ELSA includes a subset of this type of question. 


\begin{tabular}{|c|c|c|c|}
\hline TILDA & ELSA & SHARE & HRS \\
\hline Demographic Information about Respondents' Children & $\checkmark$ & $\checkmark$ & $\checkmark$ \\
\hline Financial Assistance Given to Children & & $\checkmark$ & $\checkmark$ \\
\hline Non- Financial Assistance Given to Children & $\checkmark$ & $\checkmark$ & $\checkmark$ \\
\hline Financial Assistance Received from Children & $\checkmark$ & $\checkmark$ & $\checkmark$ \\
\hline Non- Financial Assistance Received from Children & & $\checkmark$ & $\checkmark$ \\
\hline Non-Financial Help from/to Friends and Neighbours & $\checkmark$ & $\checkmark$ & $\checkmark$ \\
\hline
\end{tabular}

\section{2 (Instrumental) Activities of Daily Living and Helpers}

\subsubsection{Rationale for inclusion of this module}

As populations in both developed and developing countries continue to age, issues surrounding the support and care of older persons are receiving more attention. The risk of reduced physical ability (disability) increases with age (Manton, 1998). In many cases, this occurs in tandem with a decline in economic resources and social supports. At a policy level, it is clear that domiciliary care is prioritised under the overall aim that older people should be enabled to live in their own homes for as long as possible even after care needs arise, with the help of informal and formal services (Inter-Departmental Committee on the Care of the Aged, 1968; The Working Party on Services for the Elderly 1988).

However, there is a dearth of information on the level of dependency in the older population (extent, degree of severity), the sources of care received by the older population, the amount and recipients of care, the cost of home care versus institutional care at different dependency levels, and the sources of financing the costs of care (these shortcomings in the data are outlined in detail in Timonen et al., (2006). Moreover, little information is available on the effects of family structure on the probability of nursing home admission, the links between parental support to children and subsequent assistance from those children, and the links between help from children and the transfer of assets through bequests. There is also little information on the yearly costs of caring for older family members with cognitive impairment and/or chronic health conditions and the extent to which care givers (usually women) forgo their earning potential in order to take care of older family members.

For the purpose of assessing functional ability, respondents answer questions about their capacity to carry out a range of activities. These activities fall into two groups. "Activities of Daily Living" (ADLs) are the basic tasks of everyday life, such as eating, bathing, dressing, toileting, and moving about. Instrumental Activities of Daily Living (IADLs) are the activities performed by a person in order to live independently in a community setting, such as managing money, shopping, using the telephone, housekeeping, preparing meals, and taking medications correctly.

This section aims to estimate the extent of the care needs of the older population, the (potential) demand for, and sources of, care. One important task is to examine whether the 
patterns and forms of assistance persist over time. Increasing longevity among older persons can change the level of need for assistance, as well as the intensity of these supports. Are the providers of care the same over time? Do children who provide assistance with ADL or IADL activities tend to receive financial or in-kind support prior to or after providing such assistance? These are important questions that TILDA can answer.

\subsubsection{Measures}

The goal of this section is to establish the degree of functionality of the respondent and the help he/she receives, in the form of personal attention by a care giver, in performing his/her activities.

This section is important to measure the disability dynamics in middle aged and older persons. TILDA adopted a series of questions that have been tried and validated in other countries for measuring functionality.

This section asks respondents about any difficulties they have in the performance of all typical activities of daily living and instrumental daily living, as listed above. They are also asked about the help received with any difficulties. For analyses of informal care, all mentions of personal care and instrumental care are linked through the family roster to the specific provider, and each provider's effort is distinguished in terms of the frequency and intensity of help given. If a provider is paid, information is obtained on who paid and whether a child paid some or all of the costs; this information is also linked to specific individuals listed in the family roster. Information is also provided about whether the paid helper is Irish or non-Irish as this was of specific interest in the Irish context.

\subsubsection{Logic for choice}

In HRS and ELSA, the questions relating to the receipt of formal and informal care are connected to ability to perform ADL and IADL activities. In SHARE, these questions are in two different modules - physical health and social support. The disadvantage of the SHARE design is that although respondents are asked to indicate whether they have difficulty with (instrumental) activities of daily living (ticking all that apply), and whether anyone ever helps them with these activities (yes/no question for all (I)ADL considered together), the source of help is not queried. In contrast, the (I) ADL section in HRS and ELSA links difficulties experienced to helper(s).

The TILDA design arises from the conviction that it is more logical to start by establishing the presence of ADL / IADL difficulties, and to proceed to questions regarding the availability of help with any difficulties and the source of help (formal/informal). In the case of formal care, it is important to know who paid for or provided the care and whether a child paid some or all the cost; this information is linked to specific individuals listed in the family roster, as per HRS. 


\subsubsection{Comparability with other (longitudinal) studies}

As described above, TILDA is modelled particularly on HRS which utilises a more differentiated set of questions to reflect the types of difficulty encountered when performing both ADLs and IADLs and the source of help provided.

\begin{tabular}{|c|c|c|c|}
\hline TILDA & ELSA & SHARE & HRS \\
\hline Presence of Problems in Joints, Legs and Arms & $\checkmark$ & $\checkmark$ & $\checkmark$ \\
\hline ADL & $\checkmark$ & $\checkmark$ & $\checkmark$ \\
\hline Help with ADL and Assistive Devices & $\checkmark$ & $\checkmark$ & $\checkmark$ \\
\hline IADL & $\checkmark$ & $\checkmark$ & $\checkmark$ \\
\hline Help with IADL & $\checkmark$ & $\checkmark$ & $\checkmark$ \\
\hline $\begin{array}{l}\text { Frequency and Intensity of Help Provided, and } \\
\text { Types of Helpers (paid and non-paid helpers) }\end{array}$ & & $\checkmark$ & $\checkmark$ \\
\hline Cost of Paid Helper & & $\checkmark$ & $\checkmark$ \\
\hline Irish/non-Irish Paid Helpers & & & \\
\hline
\end{tabular}

\subsection{Transfers to and from Parents}

\subsubsection{Rationale for inclusion of this module}

The rationale for this section is discussed thoroughly in the Transfers to Children section.

This section, along with Transfers to Children and I(ADL) \& Helpers sections above, provides opportunities to model selected transfers, taking into account the preferences and constraints of the transfer donor and the circumstances of the recipient. Such data is particularly important for testing economic theories of the motivations for making transfers to others.

The decision to assist an individual family member is not made in isolation from the competing needs of other family members. For middle-aged persons, such as the majority of the TILDA sample, there is competition both within and between ascending and descending generations of the extended family. For example, in the case of a married couple, both sets of older parents may compete for a claim on the couple's resources (Wolf et al., 1997) in tandem with the need to take care of children and grandchildren.

\subsubsection{Measures}

The goal of this section is to contribute to knowledge of the characteristics and extent of help given and received within the respondent's extended family. The section refers specifically to the parents of the respondent. 
The first part deals with the demographic and socioeconomic characteristics of the respondent's parents, as well as his/her contact with them. The second part explores the monetary and inkind or non-financial assistance transferred within the extended family, particularly from the respondent and his/her siblings to the parents.

The definition of parents in TILDA comprises both biological parents and foster and adoptive parents. When asking about "parents", the questions in this section refer to the mother and/or the father if they are still married. In cases where the parents are divorced, the questions refer to the parents and (where relevant) their respective spouses. Where the respondent has been raised by parents other than biological parents, the individuals recognized by the respondent as his or her parents (who are generally the ones who provided him/her with food, education, clothing, and care during most of his/her childhood and youth) are considered as parents. In other words, we refer to the parents or guardians of the respondent.

\subsubsection{Logic for Choice}

Please see discussion in Section 3.1.3 (Transfers to Children).

\subsubsection{Comparability with other (longitudinal) studies}

Similar questions are included in SHARE and HRS.

\begin{tabular}{|c|c|c|c|}
\hline TILDA & ELSA & SHARE & HRS \\
\hline $\begin{array}{l}\text { Demographic \& Socioeconomic Characteristics } \\
\text { of the Respondent's Parents }\end{array}$ & $\checkmark$ & $\checkmark$ & $\checkmark$ \\
\hline Siblings & & $\checkmark$ & $\checkmark$ \\
\hline Financial Help to Parents & & $\checkmark$ & $\checkmark$ \\
\hline Non-financial Help to Parents & $\checkmark$ & $\checkmark$ & $\checkmark$ \\
\hline Financial Assistance Received from Parents & & $\checkmark$ & $\checkmark$ \\
\hline
\end{tabular}

\subsection{Social Connectedness}

\subsubsection{Rationale for inclusion of this module}

The protective influence of social relationships on health and well being is widely recognised in gerontology and social epidemiology research. Over the last two decades, findings from clinical/experimental studies and population studies have been remarkably consistent in demonstrating a beneficial effect of social relations on various health outcomes, including overall mortality, morbidity from a range of diseases, physical functioning, activities of daily living and cognitive decline including Alzheimer's disease and dementia. 
Gerontological and social epidemiology research has identified four domains of social engagement that are associated with health outcomes - social networks, social support, social integration and relationship quality (Cohen 1988, 2004). These domains are not strongly correlated and each of these domains influences health through different mechanisms (ibid, 2004). In order to promote policy interventions to minimize the negative effect of social isolation, there is a need to know which domain of social engagement or which combinations of domains the older person is involved or not involved in. Therefore, TILDA covers all four domains.

The extent of the respondent's social network is measured using Berkman's Social Network Index (SNI). Other domains of social engagement such as relationship quality (positive and negative social exchanges), social participation and loneliness are covered in the Self Completion Questionnaire. Social support is covered in the Transfers to Children and Transfers to Parents sections.

In addition to the SNI, we include questions on religion and spirituality. Many epidemiological studies have found that religion is significantly related to physical health (Koenig et al., 2000), mental health (George et al., 2000), and substance use disorders (Miller, 1998).

\subsubsection{Measures}

Berkman's Social Networks Index is a composite measure of four types of social connection: marital status (married versus not); sociability (number and frequency of contact with children, close relatives, and close friends); church group membership; and membership in other community organisations.

\subsubsection{Logic for choice}

The SNI is a well-validated scale for measuring social networks and has been used to predict both short- and long-term mortality, especially from cardiovascular disease (Berkman \& Syme, 1979; House et al., 1982).

\subsubsection{Comparability with other (longitudinal) studies}

Similar questions on religion are included in HRS. Although the SNI has been used in many epidemiological studies, it has not been used in ELSA, SHARE or HRS. However, these studies ask some questions about certain types of participation and sociability.

\begin{tabular}{|c|c|c|c|}
\hline TILDA & ELSA & SHARE & HRS \\
\hline Participation/Membership in Community Organizations & $\checkmark$ & $\checkmark$ & $\checkmark$ \\
\hline Sociability & $\checkmark$ & & $\checkmark$ \\
\hline Religion & & $\checkmark$ & $\checkmark$ \\
\hline
\end{tabular}



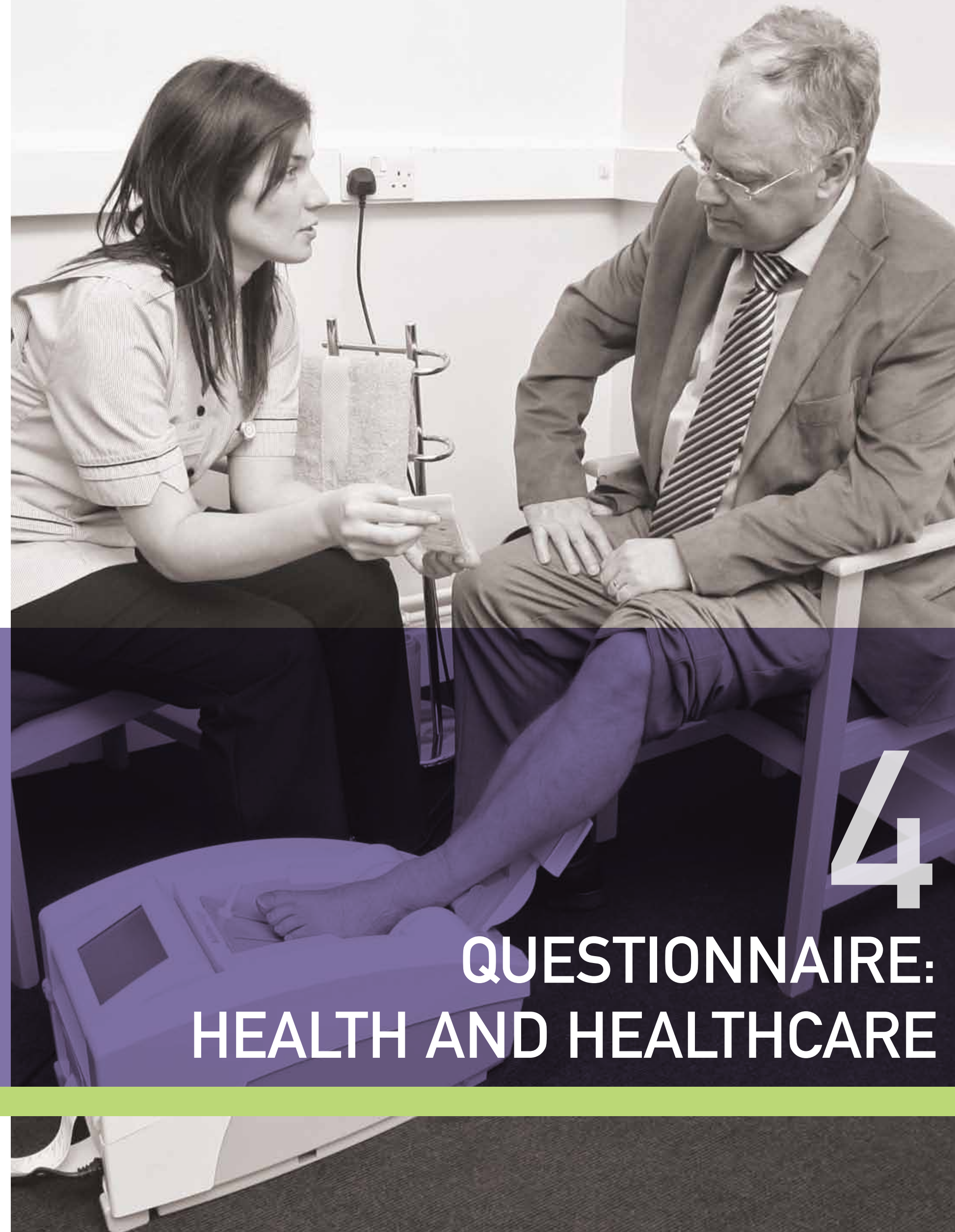


\section{4 questionnaire: health and healthcare}

\section{Introduction}

Health and wellbeing are important outcomes of interest both on their own and in relation to people's social and economic circumstances. The link between social and economic circumstances and health is well established, and TILDA will facilitate research aimed at understanding the causal mechanisms involved in these relationships. In addition to its relevance as an outcome of interest, health can influence employment patterns, economic circumstances and social participation (Smith, 1999). With its multidisciplinary focus incorporating high-quality objective and subjective measurement of health and a longitudinal design, TILDA provides a unique opportunity to understand these relationships.

The health related modules included in the CAPI are:

1. Physical \& Cognitive Health, which includes:

\section{for a. Physical Health}

1. Self-Rated Health

2. Limiting Long Standing IIIness/Disability

3. Sensory Decline

4. Cardiovascular Disease

5. Non-cardiovascular Chronic Illness

6. Falls/Fear of Falling/Steadiness/Falls History

7. Pain

8. Incontinence

9. Medical Screening

And as b. Cognitive measures:

1. Self-Rated Memory

2. Orientation

3. Word-list Learning

4. Verbal Fluency

5. Prospective Memory 
2. Mental Health, which includes:
a. Self-Reported Mental Health
b. Depression
c. Life Satisfaction

3. Behavioural Health, which includes:
a. Smoking
b. Physical Activity
c. Sleep

\section{Medications}

\section{Healthcare Utilisation}

\section{Introduction to the Health Assessment}

This last health module was included in the CAPI as a way to standardise the process by which interviewers indicate respondents' willingness to participate in a health assessment and whether they were able to set up an appointment with the TILDA administrative staff for that respondent. Interviewers may also indicate if the respondent has not yet agreed but is willing to speak to a nurse who can provide more detail. The health assessment is described in great detail in Chapter 9.

\subsection{Physical \& Cognitive Health}

\section{1a Physical Health}

\section{1a.1 Rationale for inclusion of this module}

This module in the CAPI includes both physical and cognitive health. Because these two sections are lengthy, they will be covered here separately: physical health will be described in section $4.1 \mathrm{a}$ and cognitive health will follow immediately after as $4.1 \mathrm{~b}$.

As limited information on the prevalence of chronic disease exists for the older population in Ireland, the primary focus of the physical health section is to provide detailed measures of the prevalence and incidence of disease in a community-based sample of older people. This will allow the health of older people to be monitored appropriately and provide accurate predictions for future health care needs in this age group. With constraints on interview length, TILDA includes conditions that have public health significance i.e. diseases that are most prevalent among middle-aged and elderly persons and/or which are most likely to result in morbidity, work disability and social isolation. 
Cardiovascular disease remains the main cause of death in Ireland (British Heart Foundation, 2002) and so it is a key focus of the TILDA physical health module. Research in the UK suggests that despite public health campaigns that have succeeded in reducing mortality in younger people, morbidity associated with cardiovascular disease in older age groups has not fallen and is a particular burden (Marmot et al., 2002).

Falls are a common and often devastating problem among older people, leading to significant morbidity, mortality and use of health care services including premature nursing home admissions (Health Service Executive, 2008). The risk of falling increases with age with one in three older adults falling every year and two-thirds of them falling again within six months (Gannon et al., 2007). As our population ages, it is anticipated that the number of falls and subsequent injuries in this age group will increase, therefore investigation of identifiable risk factors for falls is also a major focus of the TILDA physical health module.

\section{1a.2 Measures}

In an effort to reduce subjectivity while still using a self-report methodology, the chronic health conditions questions are designed such that respondents are asked to report health conditions where a doctor has told them that they have a particular condition. This is in lieu of just asking the respondent whether they have a particular condition. In addition, this is consistent with methods used in HRS, ELSA and SHARE and intended to encourage respondents to only report conditions that have been diagnosed by a medical doctor and exclude those based on a selfdiagnosis.

\section{1a.2.1 Self-rated health}

A large number of empirical studies have demonstrated that a person's own appraisal of his/her general health is a powerful predictor of future morbidity and mortality, even after controlling for a variety of physical, socio demographic and psycho-social health status indices (Kaplan and Camacho, 1983; Idler and Angel, 1990).

Overall physical and emotional health status is measured using five response options "excellent, very good, good, fair, or poor". In addition, respondents are asked to evaluate their physical and emotional health status compared to that of other people their own age, using the same five response options listed above.

\section{1a.2.2 Limiting long standing illness / disability}

With an ageing population and concomitant health and policy issues, it is becoming increasingly important to accurately measure the prevalence and impact of chronic illness on older people. This specific measure of population health status is likely to influence resource allocation and service delivery. It is therefore important to identify how limiting long term illness affects other aspects of health status and a person's employment and financial situation. 
TILDA employs a global question designed to measure the prevalence of chronic or long-term health problems utilised in ELSA, HRS and SHARE. Additional questions are asked to quantify the level of functional limitation associated with the chronic illness. This is important as research suggests that in older people, the impact on quality of life of functional limitation due to long term illness is more than four times greater than the impact of the long term illness itself (Netuveli et al., 2005).

\section{1a.2.3 Sensory decline}

Sensory decline represents one of the most common problems faced in later life. A substantial proportion of the older population suffers from limited vision and hearing. TILDA intends to track sensory function and decline since it is associated with a variety of negative outcomes, including reduced physical functioning, falls, increased dependency, withdrawal from social activities, and diminished quality of life (Nusbaum, 1999; Chia et al., 2006).

TILDA respondents are asked to appraise their vision (both long and short sightedness), hearing (both in individual and group settings), sense of smell and taste using the following five response options "excellent, very good, good, fair, or poor". In addition, respondents self-report eye disease and the use of assistive devices (such as glasses and hearing aids).

\section{1a.2.4 Cardiovascular disease}

Participants are asked about morbidity associated with coronary heart disease (heart attack, angina, heart failure and stroke) as well as established risk factors (such as hypertension and diabetes). Participants are asked whether a doctor has ever told them that they suffer from any of the following conditions: high blood pressure or hypertension, angina, a heart attack, congestive heart failure, an abnormal heart rhythm, diabetes or high blood sugar, a stroke, high cholesterol or any other heart trouble. Any participant who reports that they suffer from a particular condition is then asked some follow-up questions such as date of onset or level of treatment.

\section{1a.2.5 Non-Cardiovascular Chronic Illness}

Respondents are asked whether a doctor has ever told them they have or have had chronic lung disease (such as chronic bronchitis or emphysema), asthma, arthritis, osteoporosis, cancer, Parkinson's disease, psychiatric problems, alcohol or substance abuse, dementia, ulcers or cirrhosis. If the respondent reports having one or more of these diagnoses, they are then asked some follow-up questions such as date of onset or level of treatment. Respondents with arthritis are asked which type(s) of arthritis they have. Respondents are also asked questions about hip and wrist fractures and joint replacements. 


\section{1a.2.6 Falls / Fear of Falling / Steadiness}

Falls:

Respondents report history and frequency of falls in the past year, whether any of these falls were non-accidental i.e. for no apparent reason, and whether they required medical treatment. Fainting or blackouts, particularly if recurrent, have a huge impact on a person's quality of life and can lead to serious injuries (Rose et al., 2000; Ammirati et al., 2001). As the prevalence of blackouts increases in older adults, questions on blackouts and fainting have also been included in the social interview.

\section{Fear of Falling:}

Due to its association with falls and quality of life, TILDA aims to investigate prevalence and correlates of fear of falling and avoidance of activity due to fear of falling in older people. Fear of falling is measured by asking participants whether they are afraid of falling (yes/no). This assessment of fear of falling has been shown to have substantial test-retest reliability (kappa =.66) (Chia et al., 2006) but has been criticised for its limited ability to detect variability in degrees of fear (Murphy et al., 2003; Yardley, 2005). A second question which expands the answer choices to this question was therefore included to better reflect the degree of fear (e.g. "somewhat afraid," "very much afraid"). A third question on activity restriction was included to assess the impact of fear of falling on respondents' lives.

Several validated scales were considered for assessment of fear of falling in TILDA, however, their length or specificity made them unsuitable for inclusion. For example, the Falls Efficacy Scale (Tinetti et al., 1990) measures only simple indoor activities, and is therefore most appropriate for use with older adults who are housebound and have low mobility. The modified Falls Efficacy Scale (Hill et al., 1996) was excluded as it was considered too long and is similar to the Activities of Daily Living scales which are included in TILDA. The one-item fear of falling question included in TILDA has been found to correlate significantly with the Falls Efficacy Scale-International (Kempen et al., 2006).

\section{Steadiness:}

Although unsteadiness has been associated with increased falls and restricted activity in community-living elders, the prevalence and consequences of unsteadiness in older people is unknown (Lindenberger et al., 2003). Three questions on steadiness (steadiness when standing, walking, and getting up from a chair) are included in TILDA and are used to generate a steadiness score. This score is predictive of falls, hospitalisation, and decline in ADL and IADL functions (Clark et al., 2005). Improving the accuracy of disability projections is important for policy reasons, and for disability prevention.

\section{1a.2.7 Pain}

Older adults have higher rates of chronic illness and disability when compared with younger people (Marmot et al., 2002). Pain is often a central component in any chronic condition and is associated with adverse health consequences and diminished quality of life. As such, pain is of major public health concern in an ageing population and therefore questions on pain have been included in TILDA. The questions on pain in the TILDA questionnaire assess the amount 
of pain experienced, the treatment, control and location of the pain as well as the impact of pain on daily functioning.

\section{1a.2.8 Incontinence}

Urinary incontinence is not only a problem in itself but can also lead to many other problems and complications, particularly among older people. For example, incontinence can cause a person to avoid activities and interactions with others, which can lead to isolation and depression (Hajjar, 2004). In addition, incontinence can increase the risk of falls from attempts to reach the toilet quickly (Chiraelli et al., 2009).

Despite affecting millions of older people, public awareness about incontinence is minimal, probably due to the nature of the condition and the 'taboo' surrounding it. TILDA includes questions relating to the prevalence, frequency and treatment of urinary incontinence as well as any activity restriction as a result. The placement of this set of questions in the CAPI was chosen intentionally to allow interviewers to build some rapport with the respondent so that the respondents would feel as comfortable as possible answering these questions.

\section{1a.2.9 Medical Screening}

With a rapidly growing ageing population, the importance of identifying and understanding the various factors that influence health in older age is paramount. A modifiable health behavior of particular research interest is preventive health screenings. These are hugely important to older persons, as prevention and early treatment of many of the diseases and illnesses associated with such screenings can reduce morbidity and mortality among older people. The questions relating to screening included in TILDA are:

- $\quad$ Prostate Cancer Screening - PSA blood test (male respondents only)

- Breast Cancer Screening - Mammogram \& breast self exam (female respondents only)

- Cholesterol Screening

- Influenza Vaccination

\section{1a.3 Logic for choice}

The TILDA physical health measures are designed to capture the respondents' health and functional status as fully as possible, realising the constraints of survey time and resources, while being maximally responsive to the major study hypotheses and goals.

The data on physical health collected in TILDA will permit the longitudinal and long-term examination of how changes in health interact with economic, social, and psychological factors among older Irish people. 
Several criteria developed by HRS (Fisher et al., 2005) were used in designing or otherwise specifying health items, although it was clearly not possible to meet all criteria at all times:

a. High quality measures that can provide maximally valid and reliable information;

b. Proven items and instruments from the scientific literature, counterbalanced by the need to create new items responsive to modern hypotheses and conceptualisations of health, and its inter-relationship with social and economic factors;

c. Items and instruments that are at least in part represented in other recent national and international surveys in order to compare and contrast findings;

d. Measures that can be administered repeatedly \& be sensitive to subtle changes in health status;

e. Measures that are appropriate for persons of various socioeconomic backgrounds; and,

f. Level of item difficulty that is suitable for participants with high and low functional status.

\section{1a.4 Comparability with other (longitudinal) studies}

The measures of self reported physical health used in TILDA are designed to be comparable with major longitudinal studies in England, Europe and America. The sections on cardiovascular disease and non-cardiovascular chronic illness are directly comparable with ELSA and similar questions are included in HRS and SHARE.

\begin{tabular}{|c|c|c|c|}
\hline TILDA & ELSA & SHARE & HRS \\
\hline Self Rated Health & $\checkmark$ & $\checkmark$ & $\checkmark$ \\
\hline Limited Long Standing IIIness/Disability & $\checkmark$ & $\checkmark$ & $\checkmark$ \\
\hline Sensory & $\checkmark$ & $\checkmark$ & $\checkmark$ \\
\hline Cardiovascular Disease & $\checkmark$ & $\checkmark$ & $\checkmark$ \\
\hline Non-cardiovascular Chronic Illness & $\checkmark$ & $\checkmark$ & $\checkmark$ \\
\hline Falls/Fear of Falling/Steadiness & $\checkmark$ & & $\checkmark$ \\
\hline Pain & $\checkmark$ & $\checkmark$ & $\checkmark$ \\
\hline Incontinence & $\checkmark$ & & $\checkmark$ \\
\hline Medical Screening & & & $\checkmark$ \\
\hline
\end{tabular}




\section{1b Cognitive Health}

\section{1b.1 Rationale for inclusion of this module}

Along with physical decline, decline in cognitive function is a hallmark of ageing and predictive of mortality. Indeed, independence in later life is as much determined by mental ability as by physical ability (Ofstedal et al., 2005). Among middle aged and older people, a broad spectrum of cognitive capability exists with dementia at one extreme and maintained function at the other. Adequate cognitive functioning is required to perform simple Activities of Daily Living such as dressing and bathing and more complex tasks such as managing money, paying bills and taking medications (Stenn et al., 2001). Cognitive functioning is also likely to impact one's ability to work and plays a role in retirement planning and decisions around pensions and savings.

Despite its importance, the implications of declining cognitive functioning on people's daily lives as they age are complex and not well understood. One reason is the lack of adequate data sources from which to derive population-based estimates of cognitive impairment and with which to study a broad set of relevant outcome information. For these reasons, cognitive function is considered a key focus for the TILDA study.

\section{1b.2 Measures}

The cognitive module includes both memory measures and tests of executive function. The memory measures are as follows:

\section{Self-rated memory}

This measure provides an indication of whether the respondent is worried about their memory. They are asked to rate whether their memory at the present time is excellent, very good, good, fair or poor. This item comes from HRS.

\section{Orientation in time}

Knowing the day and date is a simple but effective test of memory. Time orientation is assessed by standard questions about the date (day, month, year) and the day of the week. These items are taken from the Mini-Mental State Examination (MMSE) which is used in numerous studies of ageing (Folstein et al., 1975).

\section{Word-list learning}

This is a test of verbal learning and recall, in which 10 common words are presented orally and the participant is asked to remember them. Word recall is tested both immediately and after a short delay. TILDA uses the word lists developed for HRS, which comprise four different versions, so that different lists can be given to different members of the same household. The first member of the household to be tested is assigned a list at random by the computer, and where there is more than one member of the household in the TILDA sample, the remaining lists are also selected at random. To ensure standardisation, the lists are 'read' by the computer, using a recorded voice. 


\section{Prospective memory}

Sometimes referred to as 'remembering to remember', prospective memory concerns memory for future actions. Early in the cognitive assessment section, respondents are informed about two actions that they will be asked to carry out at the appropriate time, later in the session. They are told that they will need to carry out these actions without being reminded. The first task is to remember to write their initials in the top left-hand corner of a page that the interviewer shows them, when they are later handed that page and a pen. The second task is to remember to remind the interviewer to record the time when the interviewer announces that the cognitive section is finished. When the appropriate point is reached for the respondent to carry out the actions, the interviewer waits for five seconds to see if the respondent performs the correct action without a prompt. If they fail to carry out the action spontaneously, the interviewer reminds them that they were going to do something and records what the respondent then did. A correct response requires the person to carry out the correct action without being reminded. The first task is based on a similar task used in the Medical Research Council Cognitive Function and Ageing Study (Medical Research Council CFA Study, 1998), and the second task is based on a similar task used in the Rivermead Behavioural Memory Test (Wilson et al., 1985).

\section{The test of executive function is:}

In addition to assessing memory, executive function is also assessed using word-finding or verbal fluency. This is a test of how quickly participants can think of words from a particular category, in this case naming as many different animals as possible in one minute. Successful performance on this test requires self-initiated activity, organisation and abstraction (categorising animals into groups such as domestic, wild, birds, dogs) and set-shifting (moving to a new category when no more animals come to mind from a previous category). Further objective measures of cognition are conducted in the health assessment and are described in more detail in Chapter 9.

\section{1b.3 Logic for choice}

Following guidelines from the HRS (who were the first study to assess cognitive function in the less controlled setting of a large scale national survey), TILDA's cognitive measures were selected to satisfy the following considerations:

a. provide descriptive information on a range of cognitive functions;

b. span all difficulty levels from competent cognitive functioning to cognitive impairment;

c. be sensitive to change over time;

d. be administrable in a survey environment with lay interviewers in a short time;

e. be valid and reliable; and,

f. use items and instruments that are at least in part represented in other recent national and international surveys in order to compare and contrast findings (Ofstedal et al., 2005). 


\section{1b.4 Comparability with other (longitudinal) studies}

The questions on orientation and self rated memory are directly comparable with ELSA and HRS. The word list and verbal fluency tasks are used in ELSA, HRS and SHARE. The prospective memory tasks are comparable with those used in ELSA.

\begin{tabular}{|c|c|c|c|}
\hline TILDA & ELSA & SHARE & HRS \\
\hline Self Rated Memory & $\checkmark$ & & $\checkmark$ \\
\hline Orientation & $\checkmark$ & $\checkmark$ & $\checkmark$ \\
\hline Word-list Learning & $\checkmark$ & $\checkmark$ & $\checkmark$ \\
\hline Verbal Fluency & $\checkmark$ & $\checkmark$ & $\checkmark$ \\
\hline Prospective Memory & $\checkmark$ & $\checkmark$ & \\
\hline Word-finding & $\checkmark$ & & $\checkmark$ \\
\hline
\end{tabular}

\subsection{Mental Health}

\subsubsection{Rationale for inclusion of this module}

A person's mood and emotional health is an important component of overall health and wellbeing. A high level of psychological distress is associated with poorer physical health and use of social and health care utilities. Poor mental health can have a significant impact on a person's relationships and their performance in the labour market; individuals with poor affective functioning are less attached to the labour force, work fewer hours, have more sick days, and earn lower wages (Ettner et al., 1997). Consequently, a person's emotional health is likely to affect his/her decision to retire.

For these reasons, TILDA assesses mental health as one of its health domains, including:

Self-reported Mental Health;

Depression; and,

Life Satisfaction

Depression is the most prevalent psychiatric disorder among the elderly and research suggests that it is both under-recognised and under-treated in late life (Lebowitz et al., 1997).

Depression is also associated with cardiovascular disease (Charney et al., 2003). A better understanding of late-life depression should improve diagnosis, prevention and treatment. Assessment of depression is therefore the main focus of the mental health module. Further mental health measures are included in the Self-Completion Questionnaire discussed in section 8. 


\subsubsection{Measures}

Measures of mental health in the CAPI interview include a global assessment of affective functioning, a series of questions about diagnosed mental illness, a measure of symptomatic depression and a rating of subjective life satisfaction.

\subsubsection{Self-Reported Mental Health}

The most direct method of characterising affective functioning included in TILDA is a set of questions asking the respondent to report about their own emotional health directly. This includes a global rating of emotional health as excellent, very good, good, fair, or poor (the same as in Physical Health section). Respondents are also asked directly about receipt of a diagnosis from a doctor of an emotional, nervous or psychiatric problem. This is asked within the context (on the same show card) of the non-cardiovascular chronic illnesses described in section 4.1a.2.5 above. Further questions ask whether he/she has sought psychiatric or psychological treatment for this problem. This allows the respondent to acknowledge any mental health problem for which they have sought care from a health-care professional.

\subsubsection{Depression}

The Center for Epidemiologic Studies Depression Scale (CES-D) (Radloff, 1977) was selected to assess depression in TILDA. It is designed to measure depressive symptoms in the general population (i.e. non-psychiatric persons older than 18 years). The 20 -item self-administered scale measures the major components of depressive symptomatology, including depressive mood, feelings of guilt and worthlessness, psychomotor retardation, loss of appetite and sleep disturbance. Respondents are asked to rate the frequency of these symptoms along the following categories: rarely/none of the time, some of the time, most of the time, or all of the time.

One limitation of the scale is that it does not assess suicidal ideation. Since elderly people have a higher risk of completed suicide than any other age group worldwide (O'Connell et al., 2004), it was deemed important to collect information on suicidal feelings in elderly people. The item on suicide was chosen from the EURO-D scale (Prince et al., 1999) and is asked as an individual question after the CES-D has been administered.

\subsubsection{Life satisfaction}

Life satisfaction is assessed on a 7-point Likert scale using a global one-item measure. Respondents are instructed to say how much they agree or disagree with the statement "I am satisfied with my life". Alternatives are presented on a show card ranging from (1) strongly agree to (7) strongly disagree. 


\subsubsection{Logic for choice}

The choice of mental health measures was subject to the constraints of the study format. Structured interviews by mental health providers were not feasible, so the instruments had to be designed for administration by interviewers with no advanced training in psychology or psychiatry. The instruments also had to be reliable when administered in interviewing environments similar to TILDA. An additional goal was to ensure comparability with other studies. Ultimately, the CES-D was chosen because of its widespread use among older populations, which both attests to its reliability and validity, and allows for greater comparability of the TILDA with existing research (Beekham et al., 1997).

\subsubsection{Comparability with other (longitudinal) studies}

Further self administered measures of psychological health are incorporated into the SelfCompletion Questionnaire to safeguard privacy and allow time for reflection. These include measures on quality of life, anxiety, perceived stress, worry, loneliness and a detailed evaluation of people's self perceptions of ageing.

\begin{tabular}{|c|c|c|c|}
\hline TILDA & ELSA & SHARE & HRS \\
\hline Self-reported Mental Health & $\checkmark$ & $\checkmark$ & $\checkmark$ \\
\hline Depression & $\checkmark$ & $\checkmark$ & $\checkmark$ \\
\hline Life Satisfaction & $\checkmark$ & & $\checkmark$ \\
\hline
\end{tabular}

\subsection{Behavioural Health}

\subsubsection{Rationale for inclusion of this module}

Individual behaviours can contribute to risk of disease and ill health, or promote health. With the rapidly growing ageing population, identifying and understanding the myriad factors that influence health behaviours in older age is important (Jenkins et al., 2008). The health behaviours assessed in TILDA include smoking, drinking, physical activity, and participation in preventive health screenings. The two most important negative behaviours for population health are smoking, which is declining over time, and obesity, which is increasing (ibid, 2008). The health behaviours and risk factors that are covered in the behavioural health module include:

- Smoking

- Physical Activity

- $\quad$ Sleep 
Additionally, alcohol consumption is assessed in the Self-Completion Questionnaire and preventive health screenings in the Physical Health section, described above. Body Mass Index, or BMI, as an objective measure of obesity is measured as part of the health assessment (see Chapter 9). Due to limitations on interview time and the complexity involved in ensuring an accurate measurement, dietary assessment was considered to be beyond the scope of Wave 1 of TILDA.

\subsubsection{Measures}

\subsubsection{Smoking}

TILDA's measures on smoking were developed to allow a precise lifelong smoking history to be established. Data on smoking is collected using questions on smoking status (current, past, never), age at which the participant started/stopped smoking and the average number of cigarettes cigars/cigarillos or pipes smoked per day.

\subsubsection{Physical Activity}

Physical activity is assessed in TILDA using The International Physical Activity Questionnaire (IPAQ) (Craig et al., 2003). It is a standardised measure which estimates habitual practice of physical activities. Two-forms of the IPAQ have been developed: a short and a long version, both of which involve 7-day recall of physical activity. As interview time is limited, the shortform was selected for use in TILDA. It consists of 8 items to estimate the time spent performing physical activities (moderate to vigorous) and inactivity (time spent sitting).

\subsubsection{Sleep}

TILDA includes a section on sleep as insufficient sleep has potential effects on cognition and physical functioning and therefore may increase the incidence of falls in older people. Sleep behaviour is assessed with three questions pertaining to problems falling asleep, staying asleep (duration) and frequency of daytime napping.

\subsubsection{Logic for choice}

The IPAQ was selected over other measures of physical activity as it provides an objective measure of energy expenditure. The volume of activity can be computed by weighting each type of activity by its energy requirements defined in METs to yield a score in MET-minutes. METs are multiples of the resting metabolic rate and a MET-minute is computed by multiplying the MET score of an activity by the minutes performed. MET-minute scores are equivalent to kilocalories for a 60 kilogram person. Kilocalories may be computed from MET-minutes using the following equation: MET-min x (weight in kilograms/60 kilograms) (www.ipaq.ki.se, 2005). 


\subsubsection{Comparability with other (longitudinal) studies}

In addition to providing an objective measure of energy expenditure, the IPAQ can be collapsed to provide data that is comparable with the information on physical activity collected in ELSA, HRS and SHARE. Similar to the differentiation used in the IPAQ questionnaire, ELSA, HRS and SHARE ask questions to assess participation in different types of exercise (vigorous/ moderate/mildly energetic).

\begin{tabular}{|l|c|c|c|}
\hline TILDA & ELSA & SHARE & HRS \\
\hline Smoking & $\checkmark$ & $\checkmark$ & $\checkmark$ \\
\hline Physical Activity & $\checkmark$ & $\checkmark$ & $\checkmark$ \\
\hline Sleep & $\checkmark$ & & $\checkmark$ \\
\hline
\end{tabular}

\subsection{Medications}

\subsubsection{Rationale for inclusion of this module}

Older persons tend to take more medications than their younger counterparts. The term polypharmacy generally refers to the use of multiple medications by a patient. The term is used when too many forms of medication are used by a patient, when more drugs are prescribed than is clinically warranted (Fulton, 2005), or even when all prescribed medications are clinically indicated but there are too many pills to take (pill, burden). Polypharmacy is widespread in the general population but is most common in the elderly (Haider, 2007), with some studies estimating that up to $50 \%$ of individuals age 65 years or older take an average of $\geq 5$ medications per day (Linton, 2007). Polypharmacy is associated with an increased risk of falls and fall-related injuries (Blake, 1988; Jacqmin-Gadda, 1998), delirium, decline in ADL and IADL's (Magaziner, 1989) as well as increased mortality (Alarcon, 1999). There is therefore a growing need to understand and account for the role of medications in analyses pertinent to older adult health.

TILDA will incorporate biological measures as predictors of health, social and economic outcomes so consideration of the effects of medications on both the biomeasures and outcomes of interest is required. Consequently, TILDA considers it a priority to implement an accurate system for the detailed assessment of medication use.

\subsubsection{Measures}

For medication use data, an in-home inventory of medications obtained by direct observation has been shown to be a more reliable measurement tool than self-report recall methods (Qato et al., 2008). Interviewers are therefore instructed to ask if they could see the respondent's medications so they can copy down the correct spelling for each drug. They are further instructed to record all medications that the respondent takes on a regular basis, like every 
day or every week. This is to include prescription and non-prescription medications, over-thecounter medicines, vitamins, herbal and alternative medicines.

The interviewer, who has no specialised knowledge of medications, directly records names of medications from the medication packaging (e.g., bottle, tube, blister pack) into a computerbased medication inventory within the CAPI. The inventory decided on for the capture of medications in TILDA was the Drug and Medical Appliances file (DMA) from the HSE-PCRS (Health Services Executive, 2009). This contains all the drugs reimbursable under the community schemes within Ireland. It is possible that, in some cases, the interviewer will only be able to record a medication based only on the respondent's self report (e.g., if the bottle or package is unavailable).

\subsubsection{Logic for choice}

There are many different coding systems available to capture prescribed medication. The British National Formulary (British Medical Association and Royal Pharmaceutical Society, 2009) is widely used clinically however as significant differences exist between trade names used in the UK and Ireland, it was considered unsuitable for TILDA. The World Health Organization ATC coding system is also widely used for classifying drugs. The main draw back of this system is that you have to enter the generic name of the medication, rather than the trade name (i.e. aspirin would be coded as B01AC06 - Acetylsalicylic acid. This would require the user knowing that nuseals aspirin is aspirin etc). While this may be straightforward for someone with a medical, nursing or pharmacy background, it would not be suitable for use by the social interviewers working on TILDA. A third consideration was the list of medications collated by the Irish Pharmaceutical Healthcare Association (IPHA). This list was used in the national pilot but posed many problems. Firstly, it was incomplete, with many common medications missing. Secondly, it recorded the different trade names separately, without linking back to the generic name (i.e. different trade names for aspirin recorded separately - nuseals aspirin, aspirin etc), and so the data required a lot of manipulation following collection.

There are two main advantages to the DMA system. Firstly, it is very easy for the social interviewers to administer, in that they just need to record the trade names of the medications. Secondly, as the file then automatically assigns an ATC code to every medication, subsequent analysis is much easier.

\subsubsection{Comparison with other (longitudinal) studies}

Although ELSA, HRS and SHARE collect information on drug use for specific conditions they do not record all medications taken by a respondent. The comprehensive system chosen for collection of drug use data in TILDA was modelled on the US National Social life, Health and Aging Project (NSHAP) (O'Muircheartaigh and Smith, 2007). This method of collection is also comparable with the Medical Expenditure Panel Survey (MEPS) and the National Health and Nutrition Examination Survey (NHANES) in America. 


\section{TILDA}

Medication
ELSA

SHARE

HRS

$\checkmark$ $\checkmark$

\subsection{Healthcare Utilisation}

\subsubsection{Rationale for inclusion of this module}

Population ageing has a significant effect on the efficiency, quality and cost of a country's healthcare system. It is, therefore, vital for TILDA to collect data focusing on how this influence operates. For instance, does the cost of healthcare rise proportionately with the numbers in older age groups? Are healthcare costs concentrated in the last year or two of life? What are the implications of ageing for the efficiency and equity of Ireland's mixed public/private healthcare system? What are the levels and likely trends in the utilisation of different parts of the system such as primary care, secondary care and personal social services? How is the cost burden spread across different types of individuals and households? How adequate and responsive to needs are Ireland's health and personal social services?

In order to address these and other questions, TILDA captures detailed information on respondents' use of healthcare services in the previous year and on how much the respondent spends on healthcare. This data will allow us to describe how the system operates with respect to older people and to identify its strengths and deficiencies. It will also shed light on how healthcare impacts on people's financial situation. Irish government policy makers are keen to understand whether the personal services provided by the State are appropriate and sufficient to meet the needs of the Irish population. As a result, the questionnaire asks a set of questions about the level of utilisation of these services, respondents' satisfaction with them and perceived deficiencies. Furthermore, to address a concern about low uptake of available services, the questionnaire also asks respondents how they would attempt to access new or additional services they feel they need.

\subsubsection{Measures}

This module contains three types of questions. The first is to understand the type of insurance cover respondents have, whether provided by the State or paid for privately. Specific questions therefore ask whether respondents are covered by a Full Medical Card, GP (General Practitioner) Visit Card or a private medical insurance policy. These questions are important as they will allow us to estimate the role of the public and private sector in the provision of healthcare services.

Next, we capture the respondents' use of various types of healthcare services, such as visits to their GPs; Emergency Departments; hospitals; or, nursing homes. The distinction between a respondent being admitted as a public or private patient is also captured. Specific questions relate to the way hospital visits are categorized in Ireland, as this potentially has implications for hospitals with regards their reimbursement rates for procedures, e.g. out-patient versus 
day-case procedures as compared to overnight stays in hospital and procedures that require anaesthetic. We also ask about the State healthcare services respondents avail themselves of, such as chiropodists, occupational therapists or public health nurse visits. The list of available State services was compiled to be Irish-specific based on a determination made by TILDA researchers and confirmed by government representatives. The list is meant to be exhaustive of the main personal care services available to the Irish population.

Lastly, we capture any healthcare expenses respondents might have so as to fully understand the financial impact of health care problems that arise. This includes questions that seek to understand how respondents pay for drugs, whether via Medical card, Long Term Illness scheme or Hi-Tech Drugs Scheme; Drugs Payment scheme; or via private health insurance. We also ask about any out-of-pocket health expenses in order to understand the complete financial burden of a medical issue on a person's financial situation.

\subsubsection{Logic for choice}

In general, the basic questions for this section, relating to frequency and out-of-pocket costs for GP, hospital and nursing home stays, originate in the SHARE study. The question specifically relating to the Irish Full Medical Card and GP Visit Card is from European Union Survey of Income and Living Conditions (EU-SILC) and subsequent questions about private medical cover in Ireland originate in the Health Insurance Authority 2005 Survey. Lastly, a range of Irelandspecific questions were devised by TILDA to capture respondent satisfaction with services, in line with queries and concerns put forth by the Irish government.

\subsubsection{Comparison with other (longitudinal) studies}

Several of the questions asking respondents about the number of visits they have made to a GP, the time they have spent in a nursing home, and the amount of out-of-pocket expenses for these services plus prescription medications, were used in the SHARE study. One question on the type of insurance cover a respondent has appears in EU-SILC. Lastly, the HARP study which was conducted in Northern Ireland provided wording for a question about the number of Emergency or Accident \& Emergency (A\&E) visits. This could facilitate a novel "One Island, Two Systems" analysis whereby outcomes for people with relatively similar characteristics but different health care systems can be compared. Lastly, TILDA designed some Ireland specific questions relating to satisfaction with the type, amount, and frequency of services, as well as how people would access services they were not currently receiving.

TILDA

Health Care Utilisation

\section{ELSA}

SHARE

$$
\checkmark
$$



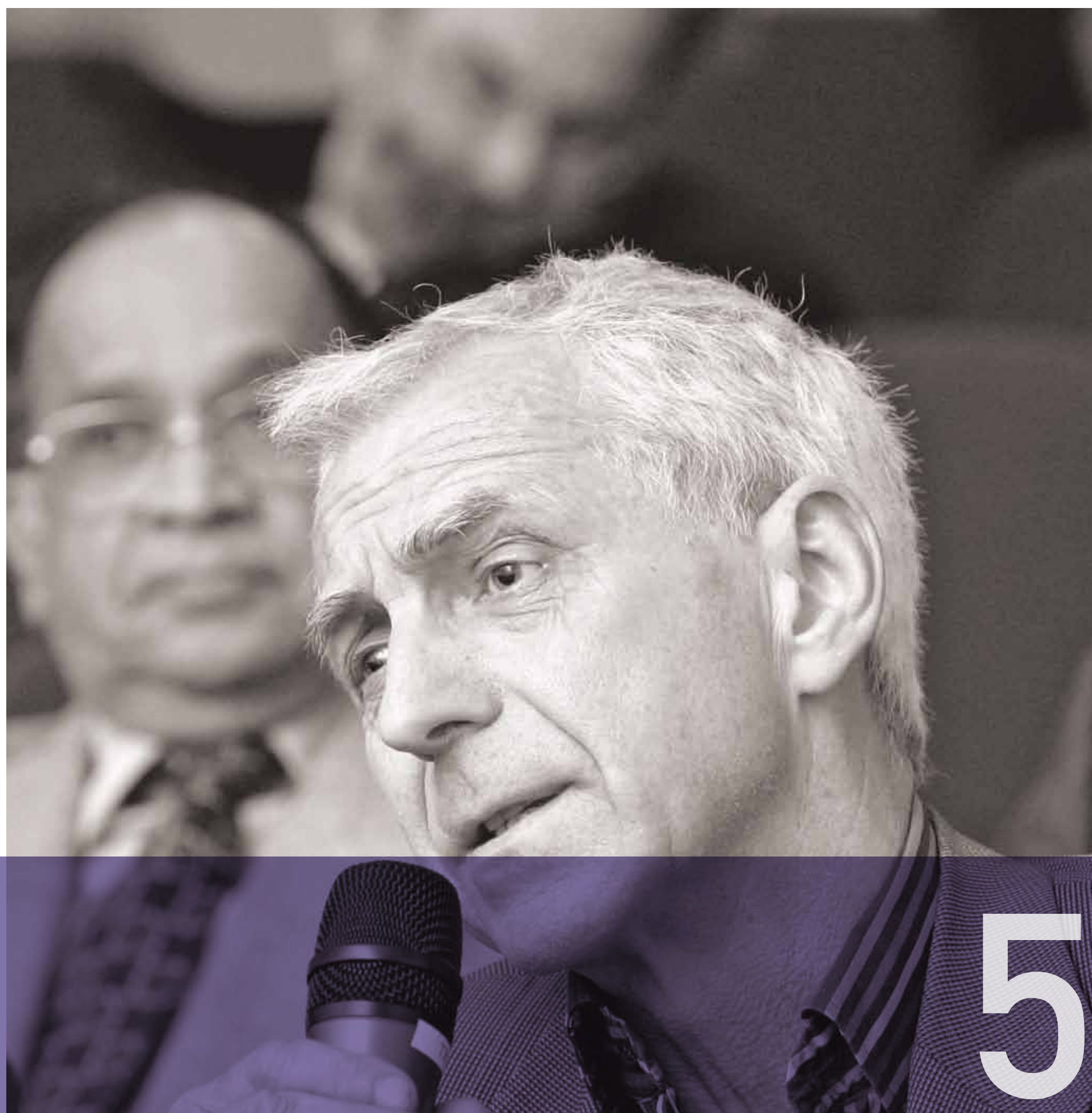

\section{QUESTIONNAIRE: \\ EMPLOYMENT AND LIFELONG LEARNING}




\section{questionnaire: employment and lifelong learning}

\section{Introduction}

The nature and extent of older people's participation in the labour market has become a key policy focus in recent years. Extending the length of working life is seen as having a vital role to play in coping with the challenges posed by population ageing. Such increases in labour force participation have at least three types of beneficial effect (Organisation for Economic Cooperation and Development, 2006):

(a) they boost labour force growth and thus offset the negative impact of population ageing on economic growth;

(b) they reduce pressure on public finances through later retirement and increased tax and pension contributions; and,

(c) they smooth the pace at which employers will have to adjust the composition of their workforce.

These priorities are also evident in the EU's Employment Policy Guidelines (2005-2008), which call on Member States to support active ageing, by promoting appropriate working conditions, improving (occupational) health status and introducing adequate incentives to work and discouraging early retirement (Commission of the European Communities, 2005).

TILDA provides key data relevant to these issues in the form of:

- an up-to-date and comprehensive portrait of the labour market participation of older people in Ireland, including the extent to which people can avail of gradual or phased retirement by moving to part-time employment;

- data on the very different experiences of older men and women in the labour market and their implications for the later stages of the life course;

- information on the extent to which older individuals represent an under-utilised labour resource in Ireland;

- information on the retirement incentives in the Irish pension system: in particular, it will allow researchers to analyse the importance of occupational and personal pensions in the individuals' decision to retire;

- data to monitor (or forecast) the impact of reforms in the pensions system, and other areas of public policy, on the labour market participation of older persons;

- information on the effectiveness of employment programmes in helping older participants in entering/returning to the labour market; 
- data on access to and participation by older persons in education, training and literacy courses, and on their impact on the individual's labour market participation;

- data on the extent and impact of age-related discrimination in the Irish labour market;

- information on how working conditions and organisational factors impact on the individuals' labour market participation; and,

- $\quad$ variables critically important for cross-domain analysis. For instance, employment status could be an important factor in determining physical or mental health. Similarly, the degree of social isolation and consequent loneliness can be affected by whether the individual is at work, unemployed or retired.

The Employment, Job History and Lifelong Learning modules in the CAPI are:

\section{Employment Situation}
a. Current activity status
e. Unemployment and
b. Main job
c. Subsidiary jobs
d. Self-Employment
Permanent Sickness/
Disability
f. Previous job

\section{Job History}

\section{Lifelong Learning}

\subsection{Employment Situation}

\section{1a Current Activity Status}

\section{1a.1 Rationale for inclusion of this module}

The questions under current activity status aim to capture information on the respondent's selfreported current activity status, his/her relationship to the labour market and whether he/she has ever worked. Employment status is a critical variable in analysing many aspects of older people's lives: standard of living, extent and nature of their contribution (through economic activity or through taking care of family), degree of dependence (ill, unemployed, etc.). A key focus of the longitudinal analysis of subsequent waves of TILDA will relate to changes over time in activity status, i.e. to the dynamics of labour force participation, including how and why people decide to retire.

\section{1a.2 Measures}

As well as asking about the respondent's (main) current status, those who classify themselves as other than in employment or self-employment are also asked if they did any work in the past week. This question is designed to collect data on people whose principal status might be retired or unemployed, but are in fact still doing some kind of paid work. 


\section{1a.3 Logic for choice}

Employment activity status is a critical variable in analysing all the domains of the TILDA study. It is important to ask these questions in order to understand the full contribution of older people to the creation of wealth in Ireland and to pinpoint the extent to which retirement can be a gradual process.

\section{1a.4 Comparability with other (longitudinal) studies}

ELSA, SHARE and HRS use similar activity status categories: Retired, Employed, Self-employed, Unemployed, Permanently Sick or Disabled, doing Care duties. Although it is not a longitudinal study on ageing, the European Union Survey of Income and Living Conditions (EU-SILC) questionnaire is well adapted to the context of the Irish labour market and its questions were heavily relied upon in the design of these sections of the TILDA CAPI questionnaire. As a result, it will be included in the comparisons in this chapter. The EU-SILC is the only study to ask if the respondent is in training or an employment programme. This category was included in TILDA so as to capture the impact of Active Labour Market Policy on older persons. With the exception of the HRS, all questionnaires ask if the respondent has ever worked in the past.

\begin{tabular}{|c|c|c|c|c|}
\hline TILDA & ELSA & EU-SILC & SHARE & HRS \\
\hline Activity Status - Self-reported & $\checkmark$ & $\checkmark$ & $\checkmark$ & $\checkmark$ \\
\hline Activity in previous week/month & $\checkmark$ & $\checkmark$ & $\checkmark$ & \\
\hline Activity Status - not working & $\checkmark$ & $\checkmark$ & $\checkmark$ & $\checkmark$ \\
\hline
\end{tabular}

\section{1b Main job}

\section{1b.1 Rationale for inclusion of this module}

Detailed information on the respondent's job is very important in understanding many aspects of the respondent's current economic and social situation. It will also be of significance in the context of longitudinal analysis of the TILDA data in examining patterns of transition between one employment status and another.

\section{1b.2 Measures}

An extensive series of questions is included relating to a person's main job: start date, type of contract, sector, occupation, hours worked, wages (gross and net), access to a company car, number of employees supervised and experience of discrimination including on age grounds. The amount of flexibility within an individual's workplace is also important as it can influence a person's decision to stay or retire from working. Questions are included to capture the amount of flexibility the respondent's employer shows towards shorter working hours or moving to less demanding jobs when an employee is nearing retirement. 


\section{1b.3 Logic for choice}

In deriving a range of questions on main job, TILDA drew on the European Foundation Survey on Working Conditions and the Quarterly National Household Survey (QNHS) Work Organisation and Working Time module. In relation to earnings, TILDA chose to follow the EU-SILC questionnaire which provided the most comprehensive coverage of wage components formulated in a manner specific to the Irish situation.

\section{1b.4 Comparability with other (longitudinal) studies}

ELSA, SHARE and HRS ask the respondent when they started their current job. ELSA, EU-SILC and HRS ask about the type of work contract the respondent has in place. SHARE in comparison seeks to differentiate between contracted hours and hours actually worked. EU-SILC asks individuals about the number of hours and days usually worked per week. It also asks the reasons for respondents working less than 30 hours per week. HRS asks about a respondent's usual number of hours worked but differentiates between a long and a short week. ELSA, EU-SILC and HRS ask about the number of weeks worked in the past 12 months. SHARE asks about months worked in the last year. ELSA and HRS ask about the number of paid leave weeks (ELSA) or days (HRS). HRS also asks about the number of paid vacation days including which months the respondent was away from work.

EU-SILC and HRS provide a more thorough analysis of the respondent's level and composition of salaries (before and after tax). SHARE, on the other hand, only asks about the individual's total salary before and after tax. In contrast with EU-SILC, all the other questionnaires use unfolding-brackets to improve response rates. All amounts are related to a specific time period (week, month, or year). The unfolding brackets technique is described in more detail in Chapter 7.

All four questionnaires ask about the respondent's employer's main activity and the number of people employed. The HRS asks more in-depth questions, e.g. downsizing processes in the company and if these can cause a threat to the respondent's job. It also asks the individuals to identify the name and address of the employer in order to inquire about employment benefits.

SHARE and especially HRS cover the job content and working conditions of respondents quite comprehensively. HRS is the only survey to ask about experiences of discrimination.

\begin{tabular}{|l|c|c|c|c|}
\hline TILDA & ELSA & EU-SILC & SHARE & HRS \\
\hline Main Job - Description & $\checkmark$ & $\checkmark$ & $\checkmark$ & $\checkmark$ \\
\hline Main Job - Working in Public/Private Sector & & & $\checkmark$ & $\checkmark$ \\
\hline Main Job - Start of Current Job & $\checkmark$ & & $\checkmark$ & $\checkmark$ \\
\hline Main Job - Type of Contract & $\checkmark$ & $\checkmark$ & & $\checkmark$ \\
\hline Main Job/Wages/Working Conditions & $\checkmark$ & $\checkmark$ & $\checkmark$ & $\checkmark$ \\
\hline
\end{tabular}




\section{1c Subsidiary Jobs}

\section{1c.1 Rationale for inclusion of this module}

In an effort to build up an accurate and complete portrait of labour market participation by older people in Ireland, respondents are asked about any second or subsidiary jobs they have.

\section{1c.2 Measures}

It would have been possible to include the same full range of questions in respect of subsidiary jobs as was done in respect of main jobs, but it was judged that this would add excessively to the respondent burden. In any case, only a minority of respondents are likely to have a subsidiary job. Brief data is collected on these jobs: whether employee or self-employed, hours worked, job title and gross earnings.

\section{1c.3 Logic for choice}

These questions collect additional data on any extra income from a second job the person might have. EU-SILC provides a more thorough analysis of the respondent's level and composition of salaries (before and after tax). TILDA chose to follow the EU-SILC questionnaire as it provided the most comprehensive coverage of wage components.

\section{1c.4 Comparability with other (longitudinal) studies}

In the second job section, SHARE repeats the same questions that were used to describe the main job. EU-SILC, ELSA and HRS inquire about hours worked. SHARE asks about contracted and actual hours worked. SHARE and HRS ask about the individual's total salary before and after tax. SHARE and HRS only ask about the individual's salary after tax.

\begin{tabular}{|l|c|c|c|c|}
\hline TILDA & ELSA & EU-SILC & SHARE & HRS \\
\hline Second Job & $\checkmark$ & $\checkmark$ & $\checkmark$ & $\checkmark$ \\
\hline Second Job - Occupation & & & $\checkmark$ & \\
\hline Second Job - Wages & $\checkmark$ & $\checkmark$ & $\checkmark$ & $\checkmark$ \\
\hline
\end{tabular}




\section{1d Self-Employment}

\section{1d.1 Rationale for inclusion of this module}

It is important to cover self-employment explicitly for a number of reasons. First, it allows the questions to be formulated in a manner meaningful to self-employed people. Second, the retirement patterns of the self-employed tend to be distinctive with a tendency for selfemployed people to retire later and sometimes on a more gradual basis than employees. In the Irish context, a high proportion of self-employment occurs in agriculture. It has been found by other surveys such as the EU-SILC and the ESRI's Living in Ireland Survey that farmers sometimes have difficulty with the precise definition of gross income and that it is advisable to ask a suite of questions relating to the details of the farm enterprise (acreage farmed, farming system, State subsidies etc.). These permit the analyst to cross-check the gross income figures given by the respondent against estimates derived from the data on the farm enterprise and national figures for income arising in agriculture.

\section{1d.2 Measures}

For the non-agricultural self-employed, the basic variables considered include: sector of activity, year business started, number of employees, size or profit or loss, tax and PRSI paid. Farmers are asked to state: the amount of land owned, rented or let, main farm enterprises, amount of State payments (Single Farm Payment, Rural Environment Protection Scheme payments, Special Areas of Conservation Payments), estimated gross farm income, weeks worked on the farm in the past 12 months, and average hours worked per week.

\section{1d.3 Logic for choice}

Understanding the nature and importance of self-employment among older people in Ireland allows us to analyse the factors that lead to lower retirement rates in this sector. The variables used should yield good estimates of income from self-employment and from farming.

\section{1d.4 Comparability with other (longitudinal) studies}

ELSA, SHARE and HRS ask about the main activity of the self-employed business. HRS also asks respondents about plans to start their own business. EU-SILC provides the most comprehensive measurement of earnings from self-employment.

\begin{tabular}{|l|c|c|c|c|}
\hline TILDA & ELSA & EU-SILC & SHARE & HRS \\
\hline Self-Employment & $\checkmark$ & $\checkmark$ & $\checkmark$ & $\checkmark$ \\
\hline Self-Employment - Farming & & $\checkmark$ & & \\
\hline
\end{tabular}




\section{1e Unemployment and Permanent Sickness/Disability}

\section{1e.1 Rationale for inclusion of this module}

To understand the labour market for older people, it is crucial to examine the causes, duration and consequences of spells of unemployment. Withdrawal from the labour market at older ages is often preceded by a spell of unemployment. It is also important to measure the extent of discouragement among unemployed older people and to examine the methods of job search that they use and how intensively they use them. The "reservation wage" i.e. the lowest wage an unemployed person will accept plays a key role in models of the labour market and a survey like TILDA allows it to be measured directly. It is also useful to document the extent of permanent illness or disability, when it impacts across the life cycle and whether it was workrelated or not.

\section{1e.2 Measures}

Among the questions included are: duration of unemployment spell, nature of the job loss (individual or collective), whether respondent is engaging in active job search, if not why not, nature of job sought and reservation wage.

\section{1e.3 Logic for choice}

TILDA collects specific data on the reasons for, and duration of, the current spell of unemployment. It also addresses the job-search behaviour of respondents. This information will be required when designing more effective policies in fighting unemployment in old age. Following HRS and SHARE, TILDA asks those whose employment status was recorded as "permanently sick/disabled" when they became disabled and whether this illness was caused by activities at work.

\section{1e.4 Comparability with other (longitudinal) studies}

HRS provides the most comprehensive set of questions relating to unemployment. It asks about the type of job sought by the respondent. It also applies the same questions to those respondents currently in work but looking for a different job. All but SHARE ask for reasons why respondents are not seeking work and the categories used in each are very similar. HRS and EU-SILC are the only surveys to ask about methods of job search. HRS enquires both about methods of job search and also type of job preferred.

\begin{tabular}{|l|c|c|c|c|}
\hline TILDA & ELSA & EU-SILC & SHARE & HRS \\
\hline Unemployed & $\checkmark$ & $\checkmark$ & $\checkmark$ & $\checkmark$ \\
\hline Reason for not seeking Work & $\checkmark$ & $\checkmark$ & & $\checkmark$ \\
\hline Method for seeking Work & & $\checkmark$ & & $\checkmark$ \\
\hline Permanently Sick/ Disabled & & & $\checkmark$ & $\checkmark$ \\
\hline
\end{tabular}




\section{1f Previous Job}

\section{1f.1 Rationale for inclusion of this module}

In order to better understand the retirement decision and the factors affecting it, we ask respondents who have already retired a series of questions about the job from which they retired and the circumstances of their retirement. These questions give insights into the factors affecting the decision to retire and so help in planning for pension schemes in the future.

\section{1f.2 Measures}

The possible questions relating to the circumstances of the respondent's retirement include: the date of retirement, why the respondent retired, whether the retirement was an early retirement, whether they received an incentive or inducement to retire and whether they retired on a full or partial pension. The questions relating to the last job held are similar to those asked in relation to current job for those respondents who are in employment. They include: length of service in the job, the occupation in question, whether it was in the public sector or not, hours worked, sector, size of firm, number supervised and net wage.

\section{1f.3 Logic for choice}

These questions permit the collection of data on retirement decisions which have already taken place. These can be contrasted with the data described in the next chapter on respondents' expectations about pensions and retirement.

\section{1f.4 Comparability with other (longitudinal) studies}

HRS does not include a question on a respondent's previous job. ELSA, SHARE and EU-SILC ask about the respondent's employment status in their last job. However, the EU-SILC typology is more comprehensive (it includes apprenticeships and participation in community employment schemes). EU-SILC inquires about the reasons why the respondent left the previous job. HRS also asks about the changes in employment that might have encouraged the worker to leave. SHARE asks about the qualifications required to perform that job and the management responsibilities of the respondent. SHARE also asks about the job title, whilst EU-SILC asks if the individual worked in the public sector.

\begin{tabular}{|c|c|c|c|c|}
\hline TILDA & ELSA & EU-SILC & SHARE & HRS \\
\hline Retirement & $\checkmark$ & & $\checkmark$ & $\checkmark$ \\
\hline $\begin{array}{l}\text { Previous Job - Occupation Type of Contract, } \\
\text { working in Public/Private sector, Hours Worked }\end{array}$ & & $\checkmark$ & $\checkmark$ & \\
\hline Previous Job - Employer & & & $\checkmark$ & \\
\hline Previous Job - Supervision & & $\checkmark$ & $\checkmark$ & \\
\hline
\end{tabular}




\subsection{Job History}

\subsubsection{Rationale for inclusion of this module}

In analysing labour market issues relating to older people, it is frequently necessary to classify the population into groups with broadly similar life-time labour market experience. Thus, for instance, one might wish to contrast women who had spent most of their working lives in employment with those who had spent the bulk of their adult life caring for the family. One way of collecting the data needed for this type of analysis would be to collect complete life history information on each respondent i.e. the date and nature of each change of labour force status. This can be quite time-consuming. A quicker, if slightly more crude, alternative is to ask each respondent to state the number of years since leaving education for the first time that they have spent in each labour force status category (e.g. at work, in education, caring for family, etc.) The CAPI software was programmed to assist in these calculations and to ensure that, for a given respondent, the total number of years given across all the labour force status categories adds approximately to the time since the respondent left full-time education. The resulting variables can then be used characterise each respondent's labour market history up to the present time.

\subsubsection{Measures}

As explained above, an alternative would be to collect a full life history for each respondent but his would be very time consuming. Therefore, the CAPI asks respondents to account broadly for the years since they first left school, identifying the number of years in each labour force status category.

\subsubsection{Logic for choice}

The final questions within this section aim to compile a job history of the respondent. TILDA included questions in this history to improve on the collection of data on how much time respondents have spent in training and education or in home duties. Their past labour market trajectory is important for TILDA to capture as it affects the individual's probability of going into unemployment, deciding to retire or to return to the labour market.

\subsubsection{Comparability with other (longitudinal) studies}

EU-SILC is the only survey to ask for a full job history (age at first job, years in work, years in unemployment, and years in other activities).

TILDA Job History

\section{ELSA}

EU-SILC

SHARE

HRS 


\subsection{Lifelong Learning}

\subsubsection{Rationale for inclusion of this module}

This module captures the frequency and type of educational and/or training activities the respondent is currently attending, or has attended in the last 12 months. The primary differentiation the questions are designed to capture is whether activities were considered formal training, leading to a higher work-related qualification, as compared with courses pursued for recreational or personal development reasons. The former help improve labour market outcomes while the latter may improve levels of social engagement and/or quality of life for people in later years.

\subsubsection{Measures}

This section inquires whether respondents have participated in any type of course of education or training in the last 12 months. They are then asked to identify the sponsoring body for that activity, for example FAS, a second level school, an institute of technology, or another body, such as a community organization. Respondents are asked about the level of the course, ranging from basic literacy to those leading to post-graduate qualifications. They are also asked to differentiate their activities into those leading to a vocational or professional qualification, specifically those courses that can impact on their employability or position, and those courses pursued for love of the task, in other words, their hobbies. Finally, they are asked to identify the number of hours and weeks attended for each type of training course.

\subsubsection{Logic for choice}

In general, the basic questions for this section are written to provide a holistic view on courses pursued by the Irish population aged 50 and over while providing comparability with another Irish source, the Quarterly National Household Survey (QNHS), conducted by the Central Statistics Office. Like the QNHS, TILDA asks people about their participation in courses, differentiating activities pursued for job-related and social reasons. TILDA added some questions about the details of the courses, such as the sponsoring body and the type of courses pursued, including whether courses were pursued to address reading or writing problems. This was to provide some insight into the successful uptake of programmes provided by the State, as requested by the Irish government. Lastly, some questions about the time spent pursuing these activities were added, which originally appear in the EU-SILC.

\subsubsection{Comparability with other (longitudinal) studies}

Most of the questions in this section were designed by TILDA. This is to reflect the specific options available in Ireland and to answer some questions of key policy interest, such as the uptake of programmes intended to address literacy and computer literacy issues. The questions about participation in courses and the reason for participating in such activities originate from the QNHS. Several of the questions relating to the time spent participating in such activities appear in the EU-SILC study. 


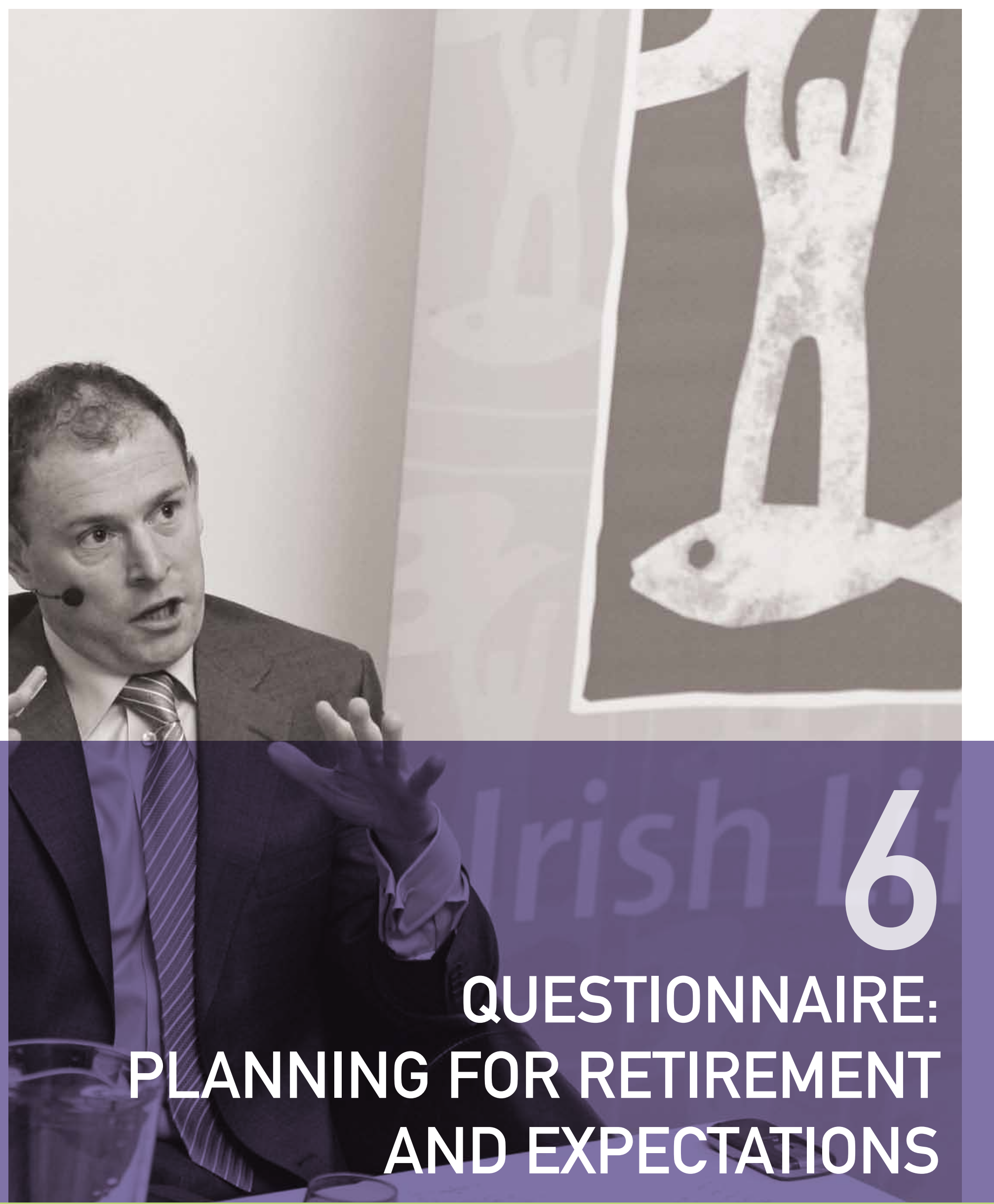

b

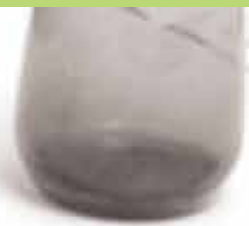




\section{6 questionnaire: planning for}

\section{Introduction}

This module deals with the arrangements people are making to provide for their retirement, while maintaining their living standards and life style. This is an area of some complexity where various organisations contribute to the possible options available to people. There is substantial involvement by the State through the social welfare system, the pension regulatory system and the taxation system. Employers are also involved through their sponsorship of occupational pension schemes, as are personal pension providers. This complexity poses problems for those planning for retirement because they must balance consideration for the timing of the retirement decision against the prospective drop in income. TILDA's longitudinal design is particularly suited to untangling the causal mechanisms involved in complex processes like this. Cross-sectional analysis can only document the end result and leaves many of the essential decision-making and behavioural aspects obscure. A full understanding of how the process operates is needed to design effective interventions.

A major policy concern for governments throughout the world is how to ensure that the overall pension system arising from this complex interaction of actors and provisions is both adequate and financially sustainable.

The Irish Government shares these priorities as outlined in the Green Paper on Pensions (Department of Social and Family Affairs, 2007).

The Green Paper summarises recent research which shows that only just over half of the workforce is covered by private or supplementary pensions. This means that more than half the population will be mainly reliant on the State pension in retirement. As the State pension is flat rate and essentially acts as a way of providing a safety net level of income support in retirement, many people could experience a marked decrease in income in retirement. In other words, the implied replacement rate will be low for many people. Population ageing will make it very difficult for the Government to make substantial increases in the State pension, thereby raising concerns about the State's ability to maintain the adequacy of the country's pension system in the long run.

It is helpful to think of the Irish pension system as being composed of three basic components or pillars: 


\begin{tabular}{|l|l|l|l|l|}
\hline Pillar & Type of Scheme & Nature of Scheme & Basis & Examples \\
\hline 1 & $\begin{array}{l}\text { State (social welfare) } \\
\text { Basic Safety Net }\end{array}$ & $\begin{array}{l}\text { Basic Contributory } \\
\text { Means-tested State }\end{array}$ & $\begin{array}{l}\text { Contributory thru PRSI } \\
\text { Pension (non-contrib.) }\end{array}$ & $\begin{array}{l}\text { PRSI State Pension } \\
\text { (contrib.) }\end{array}$ \\
\hline 2 & $\begin{array}{l}\text { Occupational } \\
\text { (employer + employee) }\end{array}$ & $\begin{array}{l}\text { Contributions } \\
\text { Defined Contribution }\end{array}$ & $\begin{array}{l}\text { Defined Benefit } \\
\text { Public Sector Scheme }\end{array}$ & Private Sector Scheme \\
\hline 3 & Personal Pension & Contributory & $\begin{array}{l}\text { Defined Contribution } \\
\text { Personal Retirement } \\
\text { Saving Account (PRSA) }\end{array}$ & Personal Pension \\
\hline
\end{tabular}

The first pillar comprises the State social welfare system which provides a range of payments such as the State Pension (contributory), the Widow(er)'s Pension etc. Entitlement to these payments is based either on contributions collected through the Pay-Related Social Insurance (PRSI) system or on a means test. Receipt of any of these payments by survey respondents is covered in TILDA's "Sources of Income" module as described in Chapter 7.

The second pillar comprises occupational pensions i.e. pension schemes organised and largely funded by employers as a benefit to their employees. These vary considerably in their organisation and benefits. For example, about one third of occupational scheme members are on a Defined Contribution (money purchase) scheme while two thirds participate on a Defined Benefit (final salary) basis. The quality of these schemes also varies in terms, for instance, of the size of the pension promised and the manner in which it is up-rated. The pensions provided for workers in the public sector are distinctive in that in most cases they operate on a "pay as you go", rather than a funded basis. They also tend to be more generous than the private sector schemes.

The third pillar covers personal pensions, i.e. pensions arranged by individuals on their own behalf through a pension provider. A special new type of personal pension called a Personal Retirement Savings Account was introduced in 2002. These schemes are standardised and their charges are capped. If an employer does not provide the staff with an occupational pension, he/she is obliged to offer employees the option of a PRSA arranged through the firm.

The information from TILDA will shed new light on a number of key policy issues:

- Pension coverage by the different types of scheme

- Variations in pension coverage by factors such as sector, occupation etc.

- Variations in coverage by gender - these are of particular significance because the historically low labour force participation rates of women in Ireland means that many women have had no opportunity to build up appreciable pension entitlements

- Reasons why some respondents have not initiated a pension

- Awareness of the pension options available

- How pensions in payment will be up-rated

- The size of the pension (regular and lump sum) respondents believe they will receive

- The size of contributions by the employee and the employer

- The extent to which respondents have assets other than pensions which could fund their retirement (property, financial assets etc.)

- As will be discussed in more detail in Chapter 7, TILDA's longitudinal design is especially suited to developing an accurate understanding of the factors underlying the retirement decision and the role prospective pensions can play. 
The Planning for Retirement module in the questionnaire is structured as follows:
a. Occupational pensions
b. Public sector pensions
c. Personal Retirement Savings Accounts (PRSAs)
d. Personal pensions plans

The Expectations module follows.

\subsection{Planning for Retirement}

\section{1a Occupational Pensions}

\section{1a.1 Rationale for inclusion of this module}

As explained above, occupational pensions are a key element in the overall Irish pension system. It is important to record as much detail as possible about them. One of the factors which is thought to have led to low levels of pension coverage is a lack of awareness of the pension options available. In order to explore this topic, special attention needs to be paid to the number of respondents giving "don't know" responses when asked about various aspects of the pension system.

\section{1a.2 Measures}

In dealing with pensions, it is common to use percentage of salary as the metric e.g. a "contribution rate of $5 \%$ of salary" or a pension equivalent to "two-thirds salary". In some cases, however, respondents may be more familiar with the money amount (especially if they have consulted a pay slip). For this reason, it was decided when asking questions with numerical answers of this kind to offer respondents the option of either giving a percentage of salary or the amount in euro.

The section begins by asking at what age the respondent plans to retire and goes on to ask whether he/she is a member of an occupational pension scheme or of a PRSA. Those who are not scheme members are asked to indicate whether they could have joined if they wished and why they did not do so. These questions are important in suggesting why policy initiatives designed to improve coverage have not been as successful as the designers hoped.

Scheme members are then asked whether enrolment was voluntary or automatic, whether early retirement is possible and at what age and on what basis they expect their pension to be up-rated or indexed. Next, respondents are asked to distinguish between Defined Contribution (DC) and Defined Benefit (DB) schemes. Respondents are then asked a series of questions on the detailed provisions of the scheme: their own and the employer's contribution, whether the respondent made any top-up payments or Additional Voluntary Contributions; how long they have been contributing; the total value of the plan at present; how much it will pay out 
on retirement in the form of lump sum and regular payment; and, whether any funds were transferred into the current pension from a previous scheme.

\section{1a.3 Logic for choice}

This information will indicate what the variations are in the nature and quality of occupational schemes in Ireland. It will also allow researchers to contrast a realistic, objective estimate of how much the pension is likely to be worth with the respondent's subjective expectation. This will provide an important indicator of how financially well prepared people are for retirement.

\section{1a.4 Comparability with other (longitudinal) studies}

ELSA and HRS provide the most comprehensive coverage of occupational pensions. Both surveys ask about the type of scheme the respondent is entitled to and the number of years in the scheme. Questions vary according to the type of scheme (DC/DB) and the specificities of the English and US pension schemes. Both surveys ask about the respondents' and the employers' contributions. They also ask questions about usual and additional contributions to plans. HRS asks to what degree it is possible to reduce contributions without losing benefits.

For respondents with DC plans, both surveys ask about value accumulated in the pension fund and expected value of pension. For those with $D B$, they ask about the expected value of the pension, whether as a lump sum or as a percentage of salary. ELSA asks about indexation mechanisms in DB schemes.

\section{TILDA}

Occupational Pensions

\section{1b Public Sector Pensions}

\section{1b.1 Rationale for inclusion of this module}

Public sector pensions have distinctive features which make it necessary to formulate the questions in a specific way. However, the basic information to be collected needs to be comparable with that collected in respect of occupational and personal pensions. For instance, it is necessary to determine the year in which respondents joined the Civil Service since there are significant differences in the pension arrangements of those who joined before and after 1995. 


\section{1b.2 Measures}

Respondents are asked what year they took up the post on which the pension is based. They then specify which of several public sector schemes they are members of. The next questions refer to payments made to enhance or top up pensions through the purchase of "added years" or through Additional Voluntary Contributions. Respondents then give their estimate of how much they will receive from the scheme as (a) a lump sum and (b) a monthly pension. Finally, they are asked how much they contribute each month.

\section{1b.3 Logic for choice}

These questions provide a picture of the nature and quality of public sector pensions which can be compared with the information on private sector schemes. As with occupational schemes, it will be possible to contrast an objective estimate of the likely value of the pension with the respondent's expectation and so assess how well-informed public sector workers are about their pension entitlements.

\section{1b.4 Comparability with other (longitudinal) studies}

HRS and ELSA ask about public sector pensions in a manner appropriate to the countries in question. SHARE groups public sector schemes along with other pillar 2 (occupational) types of scheme.

TILDA

Public Sector Pensions (with country variation)

\begin{tabular}{c|c|c|}
\hline ELSA & SHARE & HRS \\
\hline$\checkmark$ & & $\checkmark$ \\
\hline
\end{tabular}

\section{1c Personal Retirement Saving Accounts}

\section{1c.1 Rationale for inclusion of this module}

It was noted above that PRSAs were introduced in 2002 to encourage more people to make individual provision for retirement. The provisions of the PRSA schemes are standardised and their administrative charges are capped. If an employer does not provide the staff with an occupational pension, he/she is obliged to offer employees the option of a PRSA arranged through the firm. It is important for TILDA to document how effective this major policy initiative has been. 


\section{1c.2 Measures}

Similar questions to those asked of occupational scheme members were asked of those with PRSA: their own and their employer's contribution (if any); how long they have been contributing; the current value of the fund; age at which funds can be withdrawn; feasibility of early retirement; how much the respondent will receive as (a) a lump sum or (b) a pension; and, whether the respondent transferred in any funds from a previous employer's pension. In addition, PRSA holders are asked about the disposition of their fund as between equities, property, interest-bearing assets etc.

\section{1c.3 Logic for choice}

These questions are important to determine how widely used PRSAs are, how much respondents expect them to yield in the form of pensions and how well-informed people are about the PRSAs they hold. There is at present very little information available at a national level on these issues.

\section{1c.4 Comparability with other (longitudinal) studies}

As PRSAs are specific to the Irish situation, the other surveys do not have an exact equivalent.

\section{1d Personal Pension Plans}

\section{1d.1 Rationale for inclusion of this module}

These are the traditional individual pension schemes and include retirement annuity contracts. They can be held along with other types of pension scheme. They need to be included so as to provide a full picture of the extent of personal provision for retirement and of the overlap between different types of schemes.

\section{1d.2 Measures}

Again, similar questions to those asked of occupational scheme members were asked of those with private pensions: how much they contribute; how long they have been contributing; the current value of the fund; the age at which funds can be withdrawn; feasibility of early retirement; how much the respondent will receive as (a) a lump sum or (b) a pension; and, whether the respondent transferred in any funds from a previous employer's pension. Like PRSA holders, those with personal pensions are asked about the disposition of their fund as between equities, property, interest-bearing assets, etc. Respondents can be paying into more than one personal pension, so these questions are repeated for each personal scheme the respondent is a member of. The final questions in this section ask some questions about pensions from previous employments. 


\section{1d.3 Logic for choice}

Personal pensions must be included so as to give a complete picture of provision for retirement. The questions asked will provide insights into the extent of personal pension-holding and allow an evaluation of these schemes.

\section{1d.4 Comparability with other (longitudinal) studies}

SHARE and HRS use the same set of questions to inquire about occupational and personal pensions. ELSA asks about the type of scheme and number of years in the scheme. ELSA asks about regular and irregular contributions, previous contributions (and reasons for stopping), the value accumulated in the pension fund the expected benefits

TILDA

Personal Pension Plans

\section{ELSA}

HRS

\subsection{Expectations}

\subsubsection{Rationale for inclusion of this module}

People's behaviour and decisions are strongly influenced by their subjective beliefs and expectations about events in the future. For example, subjective mortality risk can influence both retirement and the desire to annuitise the wealth. It is, therefore, desirable to measure expectations about major life and labour market events.

\subsubsection{Measures}

The questions posed use a scale to measure probability in percentage terms and refer to expectations about mortality, future employment, moving into long-term care, capacity to live independently and the likelihood of experiencing serious memory problems.

\subsubsection{Logic of Choice}

Labour market participation by older people will be a key area of policy interest in the future as will people's attitude to building up savings through pensions or otherwise. Internationally, Ireland has a relatively high proportion of the population in nursing homes so it is of interest to see if this is mirrored in subjective expectations. 


\subsubsection{Comparability with other (longitudinal) studies}

Similar questions are used in the other longitudinal studies. We used the SHARE formulation of the question about future employment and the HRS questions about mortality, moving to a nursing home, living independently and memory problems.

\begin{tabular}{|l|c|c|c|}
\hline TILDA & ELSA & SHARE & HRS \\
\hline Expectations - Future Employment & $\checkmark$ & $\checkmark$ & $\checkmark$ \\
\hline Expectations - Mortality, Living Independently & $\checkmark$ & $\checkmark$ & $\checkmark$ \\
\hline
\end{tabular}



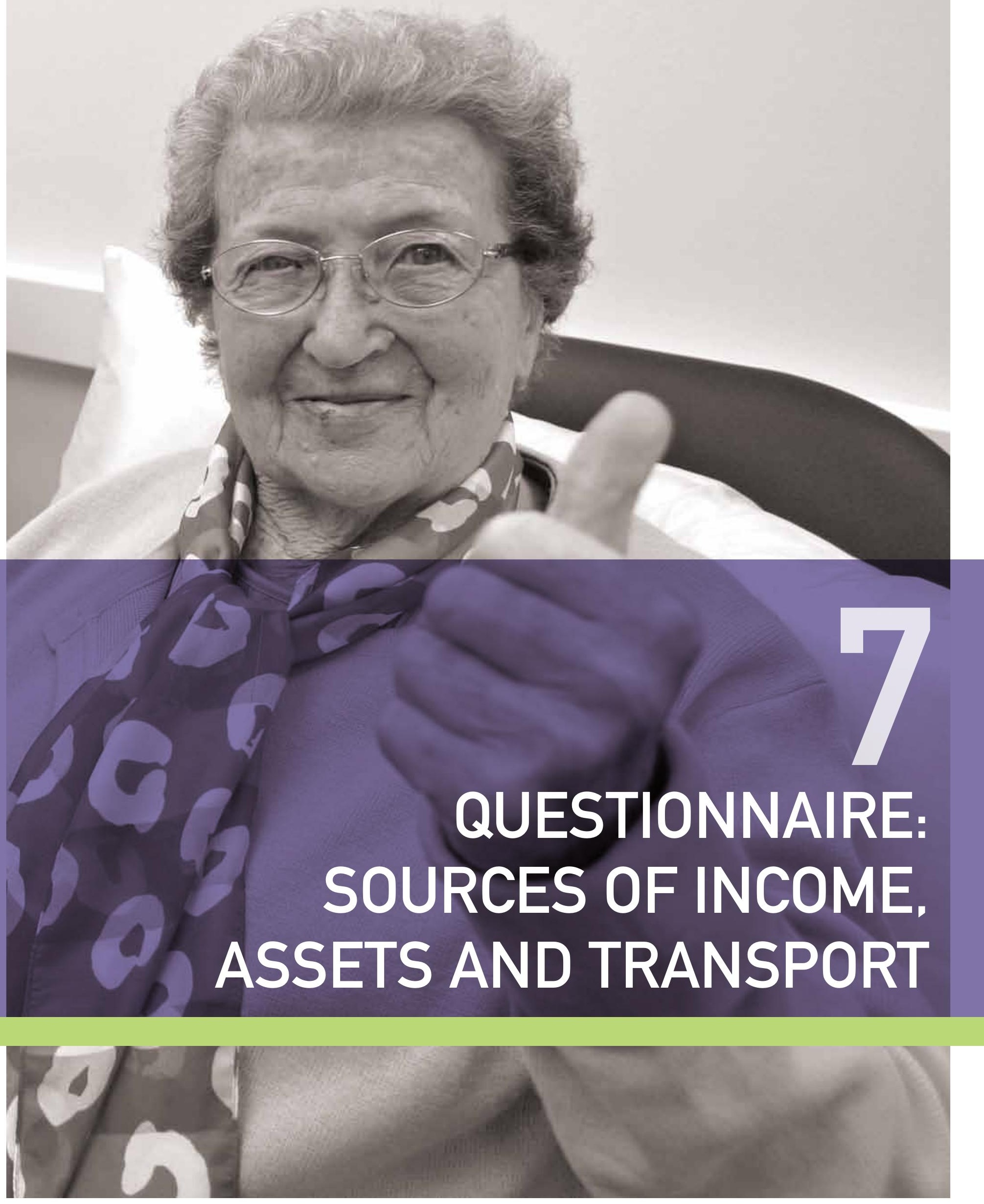


\section{7 questionnaire: sources of income, assets and transport}

\section{Introduction}

This Chapter outlines the topics covered in a number of the key economic modules of the CAPI questionnaire. It begins with the module on incomes which covers all sources of income other than income from employment which is dealt with in the Employment module in Chapter 5. It goes on to describe the data collected on home ownership and other assets and concludes by outlining the questions asked in relation to transport. Taken together, these modules aim to provide a comprehensive statistical portrait of the economic status of older people in Ireland and to permit detailed analysis and modelling of a wide range of processes and behaviour.

Questions relating to personal financial information of this kind are considered by many respondents to be highly sensitive and are prone to higher than usual levels of item nonresponse. It is, therefore, appropriate to comment at this point on the steps TILDA took at all stages of the research to minimise the problem of non-response due to sensitivity. In the first place, the documentation given to respondents deals very fully with the confidentiality of the data collected: the use of encryption in all CAPI programs, the separation of individual identifiers from substantive data in the retained database, the anonymisation techniques used and our commitment to strict adherence to data protection rules and procedures. Second, the interviewers employed on the survey are experienced field workers and have prior training and experience in dealing with sensitive questions in other surveys. Third, these issues were dealt with very fully in the interviewer training sessions and in the written interviewer instructions. Interviewers were instructed to remind respondents of the confidentiality guarantees at the beginning of sensitive sections or when respondents showed reluctance or hesitation in relation to a particular question. The importance of gaining a high level of response was continuously stressed.

A further step was taken in relation to sensitive questions concerning amounts of money. This involved the use of the "unfolding bracket" technique to handle missing data in order to reduce measurement error and lower bias (Juster and Smith, 1997). In general, this technique involves asking respondents a series of closed-ended range questions (e.g., "Is it $€ 5,000$ or more?"), after he/she has refused or said "don't know" to an open-ended question asking for the exact money amount. Using this technique, several studies have shown that relatively large proportions of respondents who initially refuse or don't know the exact answer to a financial question will answer the brackets questions. As a result, the level of item non-response can be substantially reduced and a better estimate of the income/wealth variable obtained (ibid, 1997; Kennickell, 1997; Reynolds and Ross, 1998; Hippler and Hippler, 1986; Bell, 1984). 


\subsection{Sources of Income}

\subsubsection{Criteria for inclusion of this module}

Income is a key economic variable. It can be utilised as a dependent variable measuring welfare or quality of life, as in studies of income distribution or poverty. Alternatively, it can function as an independent variable to explain variations in another variable of interest, as in studies examining the effect of income on health status. There is wide variation in the definition of income appropriate to different areas of research. A multi-purpose study like TILDA needs to provide for a number of these variants, including concept (gross and net (after tax) income); accounting period (weekly, monthly, and annual income); source (income from employment, pensions, State transfers, interest, dividends, etc.) and recipient unit (individual, couple or benefit unit, household).

Examples of the questions that the income data in the TILDA dataset needs to address are:

- what standard of living can older people in Ireland achieve and what is the extent of poverty in this population group;

- $\quad$ are pensions and asset income sufficient to offset declines in labour income;

- how could up-coming changes to the Irish pension system impact on the distribution of income and living standards of older people. (See Department of Social and Family Affairs (2007) for a review of the changes being considered);

- $\quad$ how does the distribution of income vary across different age cohorts within the older population as a result of life-time differences in education, work effort, economic opportunities, and demands on resources;

- how does the income of older households vary over time and in response to what factors; and,

- $\quad$ what are the exact conditions and provisions of the schemes at a national level. While there is some information on overall second and third pillar pension coverage in Ireland (as defined in Chapter 6, Department of Social and Family Affairs, 2007), little is known about it at a national level. A particular interest in TILDA is, therefore, to fill this gap by documenting the nature $\&$ quality of pensions being received by respondents.

\subsubsection{Measures}

TILDA contains detailed information on the annual flows of income from a variety of sources such as income from employment or self-employment (including income from subsidiary jobs); State and occupational retirement pensions; other State welfare payments; annuities; and, income arising from assets (rent, interest and dividends). These variables can be combined into indicators of personal, couple (benefit unit) and household income. 
In order to fill the gap in information about the nature and quality of pensions in Ireland, TILDA includes questions on the number of different pensions being received, whether it is a defined benefit or defined contribution scheme, the amount being received each month, information on any lump sums received and the basis on which the pension is up-rated.

\subsubsection{Logic for choice}

As well as creating a range of Ireland-specific questions based on the models from the main international ageing studies, a number of other questions were drawn from the main national source on household income, the European Union Survey of Income and Living Conditions (EU-SILC). Given the particular features of the Irish pension and social welfare systems, a number of additional questions were developed specifically for the TILDA study. These include special and more detailed treatment of income from agriculture, questions on payments from a range of State social welfare schemes and information on income accruing to respondents from the different types of second and third pillar pension schemes available in Ireland. This level of detail will permit fine analysis of the various schemes and systems and produce more accurate estimates of important aggregates such as total income, total wealth, etc.

\subsubsection{Comparability with other (longitudinal) studies}

Each of the international studies includes modules on income from different sources. Those in ELSA and HRS are specific to the pension and welfare systems of the countries in question (England and the USA) while the questions in SHARE are asked in a more general fashion. The TILDA questions are formulated to refer specifically to the Irish situation.

TILDA

Sources of Income (with country variation)

\section{ELSA}

$\checkmark$

\section{SHARE} HRS

\subsection{Assets}

\section{2a Home Ownership}

\section{2a.1 Criteria for inclusion of this module}

Housing plays an important role in determining the financial and psychological security of families and individuals as they move into their retirement years. This is the first, specific type of asset explored in TILDA. Residential real estate represents the most important asset held by Irish people aged 65 or older, as $88.9 \%$ of these people own their own dwelling (European Union Survey of Income and Living Conditions, 2001). However, while acquiring a home can boost wealth accumulation, some retired people may face unmanageable mortgage payment burdens. Even those who own their home outright (without any mortgage debt) might be 'house rich, cash poor', i.e. unwilling or unable to liquidate (some of) their housing wealth 
by downsizing. In addition, the quality of one's dwelling has an important direct role in influencing quality of life. For instance, a number of Irish dwellings lack central heating or an indoor toilet (Nolan, 2005). Poor housing quality has also been associated with mortality and ill health (Smith and Kington, 1997; Fox and Goldblatt, 1982; Evandrou and Victor, 1989; Arber and Ginn, 1993). The aim of this module is therefore to capture the details of respondents' housing conditions and the basis on which they occupy their dwellings.

\section{2a.2 Measures}

This module collects information on the type and physical features of the dwelling, ownership basis, how long occupied, estimated house value, mortgage repayments and outstanding mortgage debt and rental costs. In addition, it ascertains if there is any income arising from renting out a room in the respondent's home.

\section{2a.3 Logic for Choice}

These items were chosen so as to reflect two major aspects of housing: its overall quality and its economic importance to the household.

\section{2a.4 Comparability with other (longitudinal) studies}

All three of the international ageing studies (ELSA, HRS and SHARE) collect similar data on home ownership.

\begin{tabular}{|l|c|c|c|}
\hline TILDA & ELSA & SHARE & HRS \\
\hline Home Ownership & $\checkmark$ & $\checkmark$ & $\checkmark$ \\
\hline
\end{tabular}

\section{2b Other Assets}

\section{2b.1 Rationale for inclusion of this module}

Prospective and cross-national studies have shown that most people save for retirement (Banks, et al., 2003; Christelis et al., 2006). These assets provide income for the elderly in the form of rents from real estate, interest from saving and government bonds, dividends from stocks, and so on. The same assets can be spent down during retirement to smooth consumption or to deal with any health shocks in later life. Therefore, having adequate savings becomes important as life expectancy and health care costs increase over time. On the other hand, inadequacy of savings at retirement can lead to poverty as people will not have enough resources to finance their later consumption. Thus, the resources available to older people and their saving behaviour are central to many aspects of the current policy debate. 
An important policy issue on which very little is known in Ireland is the extent to which people accumulate assets of various kinds (real estate, financial assets etc.) as a substitute for a pension (Department of Social and Family Affairs, 2007). The answer to this question has major implications for judging how adequate the State and private pension systems are to provide a reasonable standard of living in old age.

In addition, economic status expressed by wealth is strongly correlated with the health and well-being of the older people. For example, there is much evidence that wealthier persons live longer than poorer persons. However, the direction of causality is not well understood. As Smith (1999) pointed out: "Are healthier households wealthier ones simply because higher incomes lead to better health? Or does poor health restrict a family's ability to accumulate assets by limiting work or through rising medical expenses?" Should policy makers allocate tax resources to improved health care for the less healthy or to more generous income support for the less wealthy? Understanding of this causality is important for policy makers to design policy measures to improve welfare, health and well-being of older persons in Ireland. Therefore, in addition to home ownership, TILDA asks a set of specific questions intended to inquire about each respondent's other assets.

\section{2b.2 Measures}

This module collects comprehensive information on the stock of assets owned by the household including real estate (properties other than respondent's principal residence), holdings of financial assets and car ownership. It also asks about the income flows arising from these assets in the form of interest, dividends, rents, etc.

\section{2b.3 Logic for choice}

Several questions developed by the Health and Retirement Study were adapted in designing the current module in order to capture extensive information about the economic situation of the households, including a complete accounting of assets, stocks and income flows. As only limited information on the stocks of wealth exists for the older population in Ireland, the data on assets from TILDA will provide important new information about the distribution, level and dynamics of wealth accumulation in the country.

\section{2b.4 Comparability with other (longitudinal) studies}

Each of the international studies includes questions on overall asset holdings.

TILDA

Other Assets

\section{ELSA}

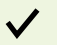

SHARE HRS

$\checkmark \checkmark \checkmark$




\subsection{Transport}

\subsubsection{Criteria for inclusion of this module}

Transportation connects individuals with economic, social, cultural and civic activities and it is essential to maintain independence, freedom and emotional well-being. For people of all ages, the private car has become one of the most important means of personal transportation, especially in rural areas. Many older people are highly dependent on the car as their main means of transport (Organization for Economic Cooperation and Development, 2001). However, many of them stop driving at some point in life, usually due to physical or mental health problems. They then become reliant on public transport or on lifts from family and friends. This can have a marked effect on their independence through its effects on many aspects of their lives: economic (getting to and from work); social (meeting relatives and friends and participating in social and cultural activities); and, health (getting to hospital appointments). In areas where public transport facilities are deficient, it may lead to increased isolation and deterioration in medical care.

\subsubsection{Measures}

This module of the TILDA questionnaire aims to assess the types of transport used by respondents; whether and how much they drive; if they have ceased driving, when they stopped driving and why; what the impact of not driving has been; the respondents' evaluation of the various types of transport (public and private) available in their area and how these could be improved.

A number of questionnaires and scales were considered including the Driving Habits Questionnaire (Vision Rehabilitation Laboratory, 2009). Most of these required too much interview time to be included. A subset of the questions from these sources was included together with some questions specially developed for the Irish situation.

\subsubsection{Logic of choice}

The TILDA questionnaire is designed to record the extent to which older people may stop driving and how driving cessation affects their social life, their ability to go about normal activities, and their ability to attend health and social care appointments. In addition, the questionnaire captures how the lack of public and private transport impacts on older people's quality of life. 


\subsubsection{Comparability with other (longitudinal) studies}

All of the three international ageing studies ask about transportation in (both public and private). HRS and ELSA also ask about driving but SHARE does not.

\begin{tabular}{|l|c|c|c|}
\hline TILDA & ELSA & SHARE & HRS \\
\hline Transportation & $\checkmark$ & $\checkmark$ & $\checkmark$ \\
\hline Driving & $\checkmark$ & & $\checkmark$ \\
\hline
\end{tabular}


SHOWS ONE BOX PER LINE WHICH
EMENT. HOW YOU FEEL ABOUT EACH

much do they really understand the way you
bout things?

nuch can you rely on them if you have a
sproblem?

nuch can you open up to them if you need to
lout your worries?

ften do they make too many demands on you?

luch do they criticise you?

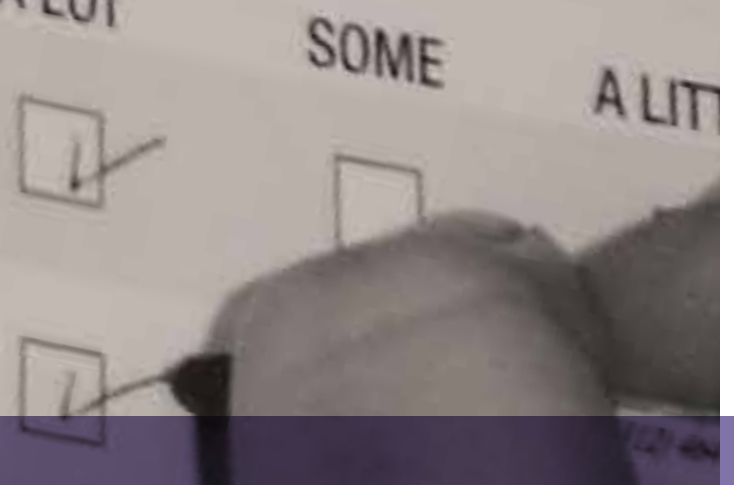

SELF-COMPLETION
uch do they let you down when you are QUESTIONNAIRE

uch do they get on your nerves? 


\section{8 self-completion questionnaire}

\section{Introduction}

All participants who complete the CAPI questionnaire are invited to fill in a paper SelfCompletion Questionnaire (SCQ). The SCQ includes questions or topics of a sensitive nature that are more suitable for completion in writing by the individual respondents. It takes approximately 15-20 minutes to complete. The participant may choose to fill in the questionnaire either while the interviewer is still in the home (i.e. if the interviewer is interviewing a spouse or family member) or at another time and return it by post.

The topics included in the SCQ are:

1. Social Connectedness, which includes:

a. Participation in Social / Recreational Activities

b. Relationship Quality

2. Loneliness

3. Perceived Stress

4. Stressful Life Events

5. Anxiety

6. Worry

7. Quality of Life

8. Ageing Perceptions

9. Alcohol

The questions contained in the SCQ complement the information collected in the main interview. The questions on social participation, relationship quality and loneliness contained in the SCQ complement the 'Social Connectedness' section in the CAPI questionnaire. The questions on stress, anxiety and worry complement the mental health section in the main questionnaire. In addition, we have included a quality of life measure developed for use in early old age. The SCQ also contains questions on alcohol intake which completes the main topics in behavioural health, when it is combined with the answers from that section of the CAPI questionnaire. 


\subsection{Social Connectedness}

The questions on social participation, relationship quality and loneliness aim to generate a comprehensive picture of older adults' social engagement and 'connectedness'. According to Mendes de Leon (2005), 'there is a growing recognition that social engagement is important for successful ageing' (p.64), commonly conceptualised as health and wellbeing. However, as Bath and Deeg (2005) point out, 'the studies to date have been conducted only in the US and Scandinavia, and it is not clear whether the benefits associated with social participation are also experienced among older people in other countries' (p.29). The questions pertaining to social engagement are therefore essential for performing many of the analyses focused on the interaction of social and health variables in the Irish and comparative context. For example, it will help us understand whether or not social engagement impacts on objective and subjective health over time.

\section{1a Participation in social/recreational activities}

\section{1a.1 Rationale for inclusion of this module}

While many studies point towards the beneficial impact of social and recreational activities on health and wellbeing, Maier and Klumb (2005) highlight that little work has been done to date on the possible differential impact of types of activity. In one of the few examples of categorising social activities, Lennartsson and Silverstein (2001) used principal components analysis to divide activities into social-friendship (e.g. visiting friends), social-cultural (e.g. going to cultural events), solitary-sedentary (e.g. reading books) and solitary-active (e.g. working in the garden). Even activities that require little or no physical exertion have been shown to improve survival, even when controlling for socio-demographic and health variables (Glass, et al., 1999). There is substantial literature linking social participation and health inequalities and this is an area of interest for TILDA.

\section{1a.2 Measures}

Questions on participation and frequency of both structured and unstructured activities are included in TILDA. Under structured activities we understand activities such as attending classes, participating in sport activities or exercise, and undertaking voluntary activities. Unstructured activities comprise activities which are either active (visiting people, reading a book, etc.) or passive activities (watching television, listening to music or the radio). In addition, a question on voting participation is also asked.

\section{1a.3 Logic for choice}

It is important to capture a wide range of social and recreational activities, both more structured and informal ones, in order to obtain a comprehensive picture of different types of activities and to progress the categorisation of activities. The questions included in the 
SCQ capture both active pursuits usually undertaken together with other people (for example playing cards, eating out) and more passive activities such as watching television. The list of questions also encompasses both structured and informal activities: the former includes, for instance, doing voluntary work, and the latter includes going to the pub. Voting participation has been used as a measure of social capital. In order to capture voting activity, we ask participants if they voted in the last general election.

\section{1a.4 Comparability with other (longitudinal) studies}

ELSA, SHARE and HRS capture organised, structured activities. In addition, ELSA and HRS capture informal activities.

\begin{tabular}{|l|c|c|c|}
\hline TILDA & ELSA & SHARE & HRS \\
\hline Organized, Structured Activities & $\checkmark$ & $\checkmark$ & $\checkmark$ \\
\hline Informal Activities & $\checkmark$ & & $\checkmark$ \\
\hline
\end{tabular}

\section{1b Relationship Quality}

\section{1b.1 Rationale for inclusion of this module}

The quality of family and other intimate relationships is often emphasised as a sustaining force in Irish society. The set of questions on relationship quality in TILDA will enable us to establish, for the first time in Ireland, the perceptions among older people of these relationships. Information on relationship quality will also help in constructing a variable that will enable us to control for the quality of relationships when examining, for instance, the impact of relationships and social networks on health.

\section{1b.2 Measures}

Relationship quality is measured by 7 items of positive and negative interactions provided by a spouse, children, relatives and friends. Positive interactions with a spouse, children, relatives and friends are measured by asking respondents the following three questions:
a) How much they feel their spouse/partner (children/relatives/friends) understand(s) their feelings;
b) How much they can rely on their spouse/partner (children/relatives/friends) if they have a serious problem; and,
c) How much they can open up to their spouse/partner (children/relatives/friends) if they need to talk.

In contrast, negative interactions with a spouse, children, relatives and friends are measured by asking respondents the following four questions: 
a) How much does/do their spouse/partner (children/relatives/friends) make too many demands on them;

b) How much does/do their spouse/partner (children/relatives/friends) criticise them;

c) How much does/do their spouse/partner (children/relatives/friends) let them down when they are counting on them?

d) How much does/do their spouse/partner (children/relatives/friends) get on their nerves?

\section{1b.3 Logic for choice}

Seven questions refer to the positive interactions and negative interactions that participants might have experienced with their partner/spouse, children, other relatives and friends. These are important to understand social factors that impact a person's quality, and possibly length, of life.

\section{1b.4 Comparability with other (longitudinal) studies}

Both ELSA and HRS include similar questions on relationship quality.

TILDA

Relationship Quality

\section{ELSA}

SHARE
HRS

\subsection{Loneliness}

\subsubsection{Rationale for inclusion of this module}

Although social isolation is sometimes equated with loneliness, loneliness and social isolation are separate concepts and are not necessarily correlated. Social isolation is related to objective characteristics and refers to the absence of relationships. In contrast, loneliness pertains 'to the feeling of missing intimate relationships or missing a wider network', and is therefore 'an individual's subjective evaluation of his or her social participation or isolation' (de Jong et al., 2004). Loneliness scores predict a wide variety of mental and physical health outcomes, such as depression, nursing home admission and mortality.

There is a paucity of major data sources on loneliness and social isolation among the older population of Ireland. The only comprehensive study on social isolation and loneliness was commissioned by the NCAOP (National Council on Ageing and Older People) in 2004. This study has several limitations. First, the data was collected through telephone interviews which may result in more conservative outcomes, since, for instance, cognitively impaired older persons are likely to be excluded from the sample. Second, this study collected no information on ADL or IADL impairments, sensory impairment, chronic disease, depression, anxiety, personality traits or cognitive function, which are potential risk factors associated with social isolation and 
loneliness. Third, it is likely that social desirability bias may have influenced the study findings, as people may over-report their levels of social engagement to others. TILDA will avoid these limitations by collecting information on loneliness in the SCQ and by linking this data with key social, economic and health dimensions from the other components of the study over time. Moreover, unlike the NCAOP study which was cross-sectional, TILDA is longitudinal and will be able to provide both a snapshot of the social isolation and loneliness of the respondents as well as trends about how they change over time.

\subsubsection{Measures}

Loneliness is assessed using 5 items from the University of California-Los Angeles (UCLA) Loneliness scale, in particular selecting 4 negatively-worded questions (How often do you feel left out?) and one positively-worded one (How often do you feel in tune with the people around you?).

\subsubsection{Logic for choice}

The UCLA Loneliness Scale was developed to assess subjective feelings of loneliness or social isolation and it is the most widely used measure of loneliness in the international literature. The dominant factor underlying the UCLA Loneliness scale is 'perceived social isolation' (Russell et al., 1980; Austin, 1983; Knight et al., 1988; Russell, 1996).

\subsubsection{Comparability with other (longitudinal) studies}

Both ELSA and HRS include the revised version of the UCLA scale, the R-UCLA scale, so TILDA's measure of loneliness is directly comparable to these international studies.

\begin{tabular}{|l|c|c|c|}
\hline TILDA & ELSA & SHARE & HRS \\
\hline R-UCLA & $\checkmark$ & $\checkmark$ & $\checkmark$ \\
\hline
\end{tabular}

\subsection{Perceived Stress}

\subsubsection{Rationale for inclusion of this module}

The objective of TILDA is to measure both subjective (perceived) and objective stress (stressful life events) as they have been implicated as determinants of health and well-being. 


\subsubsection{Measures}

The 4-item short form of the Perceived Stress Scale was selected. The PSS-4 poses general queries about relatively current levels of stress experienced. All items begin with the same phrase: In the last month, how often have you felt...? Questions ask about respondents perception of being able to control the important things in their lives; their confidence in being able to handle their personal problems; whether things were going their way; and, if difficulties were piling up so high that they could not overcome them.

\subsubsection{Logic for choice}

The Perceived Stress Scale (PSS-4) measures the degree to which situations in one's life over the past month are appraised as stressful (Cohen et al., 1983). Items were designed to detect how unpredictable, uncontrollable, and overloaded respondents find their lives. The PSS-4 poses general queries about relatively current levels of stress experienced.

\subsubsection{Comparability with other (longitudinal) studies}

As both ELSA and HRS contain the PSS-4 scale, TILDA's measure of perceived stress is directly comparable.

TILDA

PSS-4

\section{ELSA}

SHARE
HRS

\subsection{Stressful Life Events}

\subsubsection{Rationale for inclusion of this module}

There is a substantial and growing body of literature linking perceived stress and stressful life events to physical and mental illness and mortality (Rosengren et al., 1993; Leserman, 2000). For example, stressful life events have been linked to psychiatric symptoms, depression as well as heart disease and cancer. As such, these are important to document in TILDA.

\subsubsection{Measures}

The Stressful Life Events is a 12-item self-report measure to assess lifetime exposure to traumatic events such as a life-threatening accidents, life-threatening illness, death of a child, etc. For each event, respondents are asked to indicate whether the event occurred ("yes" or "no"), and the year in which the event occurred. 


\subsubsection{Logic for choice}

The questions on stressful life events assess "traumatic" events (usually life-threatening or otherwise threatening to physical integrity), and other major events (e.g., spousal bereavement) over the life course of the respondents. These can be used to understand health status as well as length and quality of life.

\subsubsection{Comparability with other (longitudinal) studies}

The questions asked in TILDA are directly comparable with the HRS.

TILDA

Stressful Life Events
ELSA

SHARE
HRS

\subsection{Anxiety}

\subsubsection{Rationale for inclusion of this module}

Epidemiologic studies regarding psychopathology in later life have mainly concentrated on depression and dementia. In comparison, anxiety disorders occurring in the elderly have received far less attention. The community-based studies conducted so far have resulted in widely differing prevalence rates while systematic exploration of the risk factors associated with late-life anxiety disorders is under-represented in the literature. The evidence so far shows that, although less common than among younger adults, anxiety disorders remain highly prevalent among older people.

\subsubsection{Measures}

The Hospital Anxiety Depression Scale - Anxiety subscale (HADS-A) was selected for use in TILDA (Zigmond and Snaith, 1983) because it has been used on non-psychiatric patients and it excludes somatic symptoms, which are perceived physical manifestations of what is in fact a mental illness. Several other scales were considered by the neuro-psychological working group including the Hamilton Anxiety Rating Scale (HAM-A) (Hamilton, 1959), the State-Trait Anxiety Inventory for Adults (Hodges and Spielberger, 1969; Kvaal et al., 2005), and the Geriatric Anxiety Inventory (Pachana et al., 2007). However, as these scales have been developed primarily for use in psychiatric patients and rely heavily on somatic symptoms (that could confound results) they were deemed unsuitable for inclusion in TILDA. 


\subsubsection{Logic for choice}

The HADS-A is a widely-used 7-item self-report scale designed to briefly measure current anxiety symptomatology in non-psychiatric hospital patients. Its main advantage over the other scales is that it excludes somatic symptoms, therefore avoiding potential confounding by somatic symptoms. It is also quick and easy to administer.

\subsubsection{Comparability with other (longitudinal) studies}

Both the Longitudinal Ageing Study Amsterdam (LASA) and the Nottingham Longitudinal Study of Activity and Ageing UK have used HADS-A to measure anxiety.

\subsection{Worry}

\subsubsection{Rationale for inclusion of this module}

Worry is a universal experience, but a clear understanding of this phenomenon remains elusive. Worry is associated with all of the anxiety disorders (Barlow, 1988; Craske et al., 1989) and is the central feature of Generalised Anxiety Disorder (GAD). Determining the nature and function of worry is an important goal for TILDA if we are to understand the human experience of anxiety and depression.

TILDA is also interested in investigating the association between cardiovascular disease and worry. Recent longitudinal investigations in the US (Normative Aging Study) suggest that high levels of worry may increase the risk of coronary heart disease. In addition, daily worry is related to low heart rate variability, a surrogate marker for autonomic dysfunction (Brosschot et al., 2007). Both stress and anxiety are already well established risk factors for cardiovascular (CV) disease. One hypothesis that TILDA is keen to explore in more detail is whether worry may be the common pathway linking stress, anxiety and cardiovascular disease.

\subsubsection{Measures}

The 8-item short form of the Penn State Worry Questionnaire was selected for use in TILDA. The respondent is presented with eight statements, such as "my worries overwhelm me", and "I was always a worrier" and scores their response on a Likert scale from 1 (not at all typical) to 5 (entirely typical).

\subsubsection{Logic for choice}

The Penn State Worry Questionnaire (Meyer et al., 1990) was selected as it is the most widely utilised, reliable and valid measure of worry. This 8-item inventory is designed to capture the generality, excessiveness, and uncontrollability dimensions of pathological worry. 


\subsubsection{Comparability with other (longitudinal) studies}

This measure is a novel addition to longitudinal studies.

\subsection{Quality of Life}

\subsubsection{Rationale for inclusion of this module}

Now that people are living longer, it is important to establish the extent to which these gains in life represent gains in quality of life (QOL). As an outcome measure, QOL is a subject of interest for social, economic and health researchers. In addition, policymakers are becoming interested in the ways in which older people define their own quality of life and the value that they place on the different domains assessed.

\subsubsection{Measures}

CASP-19 was selected to measure QOL in TILDA. Concepts assessed are Control, Autonomy, Self-actualisation and Pleasure (CASP). The scale has 19 items, is self-administered and has early evidence of good psychometric properties (Hyde et al., 2003; Netuveli et al., 2006). The complete set of questions is listed in Appendix 2.

The other scale considered to assess QOL was the short version of the WHOQOL - the WHOQOL-Bref (World Health Organisation, 1998). Its focus is on four major life domains physical health, psychological health, social relationships and environment. Each of these issues is already addressed in detail in TILDA, with the exception of positive aspects of psychological well-being. Hence, the CASP-19 provides more complementary information to the other priorities of TILDA and provides comparisons, albeit within ageing groups, with other national studies of older people.

\subsubsection{Logic for choice}

The CASP-19 scale was developed with an older population in mind and, unlike many QOL instruments, measures qualities distinct from health (ibid., 2006). It is based on a needs satisfaction model of QOL in early old age and has four ontologically-grounded domains of need.

\subsubsection{Comparability with other (longitudinal) studies}

Both ELSA and HRS use CASP-19 while SHARE uses a modified version (CASP-12). The Whitehall II and British Household Panel Survey also use CASP-19 as their QOL measure. 


\begin{tabular}{|l|c|c|c|}
\hline TILDA & ELSA & SHARE & HRS \\
\hline CASP-19 & $\checkmark$ & & $\checkmark$ \\
\hline CASP-12 & & $\checkmark$ & \\
\hline
\end{tabular}

\subsection{Ageing Perceptions}

\subsubsection{Rationale for inclusion of this module}

The relationship between self-perceptions of ageing and health-related outcomes has been documented through an array of qualitative and quantitative studies. TILDA therefore considers the direct evaluation of people's own experiences of ageing to be important, in parallel to assessing age-related profiles and changes (e.g. in cognition, cholesterol etc.) which are assessed outside of the interpretation or experience of the older persons themselves.

It is likely that the experience of ageing is very much shaped by factors such as expectations, environment, socioeconomic status, health, and societal attitudes. As a result, one would expect to see much variety in an individual's experience. Further, the experience of ageing might change as society adapts to an increasing proportion of older people. In international research, self-perceptions of ageing have been implicated as independent predictors of functional disability and death in older adults.

\subsubsection{Measures}

The Ageing Perceptions Questionnaire (APQ) was selected to measure the experience of ageing in TILDA. The full questionnaire and its scoring key (which is obviously not shared with respondents but is shown here to indicate which items refer to which categories of inquiry) are included in Appendix 3. The APQ assesses self-perceptions of ageing along eight distinct domains; seven of these examine views about own ageing: timeline chronic, timeline cyclical, consequences positive, consequences negative, control positive, control negative, and emotional representations (32 questions); the eighth domain is the identity domain and this examines the experience of health-related changes (17 health issues).

\subsubsection{Logic for choice}

The Ageing Perceptions Questionnaire is a theoretically-derived instrument with sound psychometric properties that has been developed and validated by researchers at the Royal College of Surgeons Ireland (Barker et al., 2007).

\subsubsection{Comparability with other (longitudinal) studies}

The APQ was used in the Irish Health and Social Services for Older People II study (HeSSOP II). 


\subsection{Alcohol}

\subsubsection{Rationale for inclusion of this module}

Ireland has one of the highest levels of alcohol consumption in the European Union. Within our society, alcohol consumption is accepted as legally and culturally normative. While there are some advantages associated with moderate consumption, it is also clear that alcohol consumption contributes to an array of personal and social difficulties, both of an acute and a long-term nature. While regular consumption of even moderate amounts of alcohol may create a health risk, the pattern of binge drinking (substantial intake during a single episode of drinking interspersed with periods of low or no intake) carries with it a particularly high risk of behavioural and health difficulties.

\subsubsection{Measures}

TILDA's measures on alcohol were developed in order to examine drinking habits as well as binge drinking. Participants are asked about the frequency of alcohol consumption as well as the quantity of alcohol consumed within the preceding six months.

The CAGE questionnaire, the name of which is an acronym of its four questions, is a widely used method of screening for alcoholism. The respondent is asked the following four questions:

1. Have you ever felt you needed to Cut down on your drinking?

2. Have people Annoyed you by criticizing your drinking?

3. Have you ever felt Guilty about drinking?

4. Have you ever felt you needed a drink first thing in the morning (Eye-opener) to steady your nerves or to get rid of a hangover?

\subsubsection{Logic for choice}

The CAGE questionnaire has been extensively validated for use in identifying alcoholism (Mayfield et al., 1974; Kitchens, 1994). CAGE is considered a validated screening technique, with one study determining that CAGE test scores >=2 (each 'Yes' answer scores 1 point) had a sensitivity of $93 \%$ and a specificity of $76 \%$ for the identification of problem drinkers (Bernadt et al., 1982). 


\subsubsection{Comparability with other (longitudinal) studies}

Most longitudinal studies of ageing ask about alcohol intake and behaviours surrounding alcohol. These include ELSA, HRS and SHARE. In addition the Irish national health survey SLAN 2007 contains data on alcohol and patterns of drinking in Ireland.

TILDA

Alcohol

\section{ELSA}

$\checkmark$

\section{SHARE}

HRS 


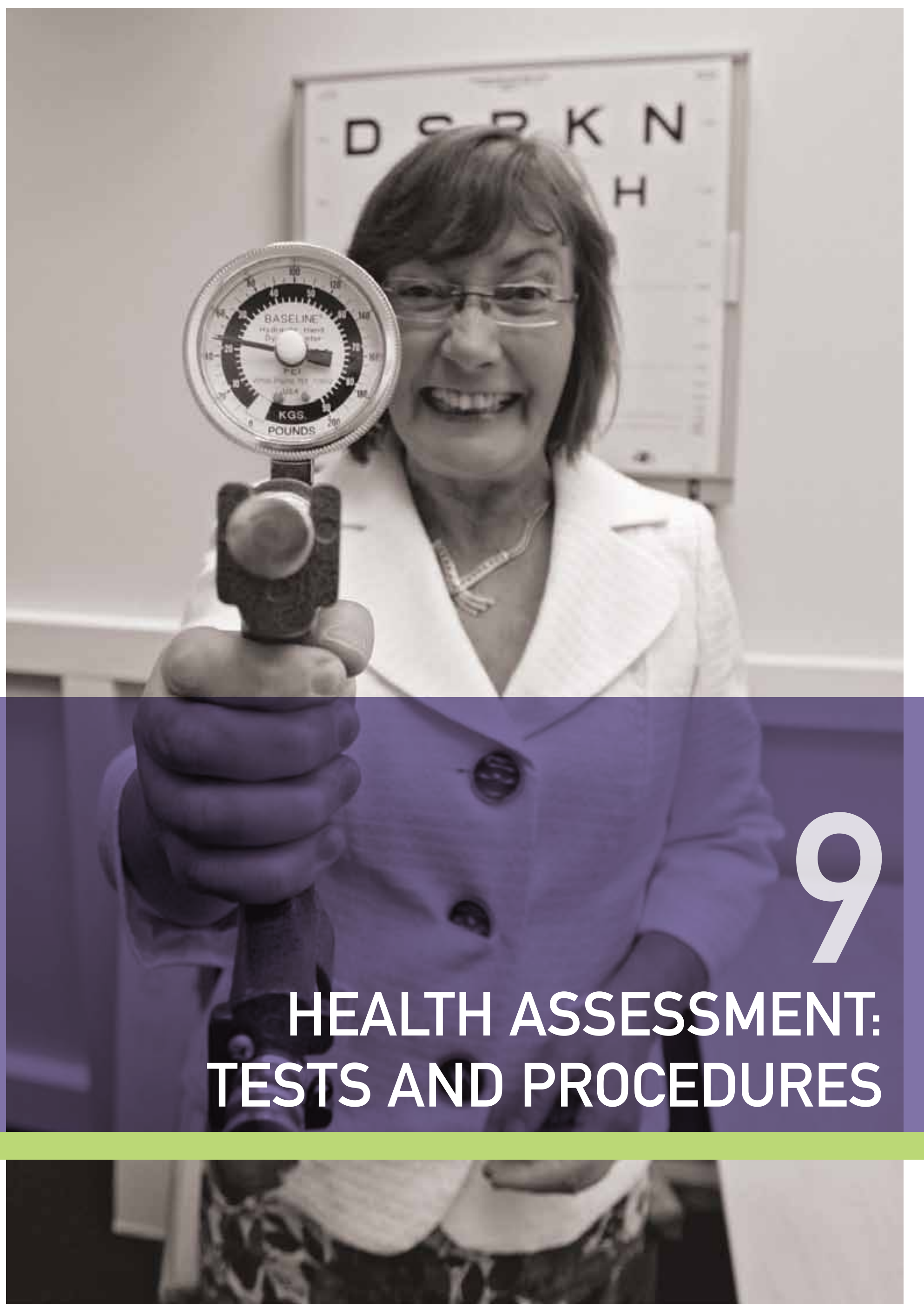




\section{0 health assessment: tests and procedures}

\section{Introduction}

All respondents who complete the CAPI interview are invited to participate in a health assessment in one of two dedicated health centres, in Dublin or Cork, or in their own home. The two dedicated centres were set up in areas chosen to reflect the greatest population density in Ireland. The health assessment for the first wave of data collection for TILDA is a combination of initial planning and changes resulting from two pilots conducted early in the fieldwork, (which will be described later and in the next chapter). The health and functioning measures collected in the CAPI interview and by the self completion questionnaire are selfreport (with the exception of a number of memory and cognitive function tests). In order to fully describe the health and well-being of older people, both questionnaire and physical measurements are required. The health assessment complements the CAPI and self-completion questionnaires by adding a range of objective measures of health status and functioning, including the collection of venous blood.

The objective data from the TILDA health assessment, in combination with the questionnaire data, will create a comprehensive research resource. This resource can be used to gain a better understanding of how the multiple aspects of health and well-being contribute to successful ageing and to identify factors that predict the development of disease. The availability of such a large and complex dataset will facilitate other research studies including nested case-control studies.

The main focus of the TILDA health assessments is on neuro-cardiovascular instability, gait and balance disorders and age-related macular degeneration.

This chapter focuses on issues related to the TILDA health assessment including:

1. Development of the Health Assessment

2. Pilot Outcomes

3. Health Assessments: Centre-based and Home-based

4. Blood Collection 


\subsection{Development of the Health Assessment}

\subsubsection{Working Groups}

The development of measures for the TILDA health assessment was coordinated by a number of TILDA Health Working Groups which drew on the expertise of psychologists, gerontologists, cardiologists, physiotherapists, ophthalmologists, nurses, geneticists and psychiatrists (see Appendix 4 for a list of Working Group members).

The TILDA health team prepared extensive literature reviews for each working group. Measurements and findings from international longitudinal studies were examined and key research questions identified.

\subsubsection{Research Questions}

Working Group-specific research questions and individual level research questions were developed at this stage along with the research questions guiding the collection of data in the whole TILDA health domain.

The overarching TILDA health-related research questions are:

1. What changes occur in physical, psychological and cognitive function over time and across ages?

2. What are the determinants of changes in physical, psychological and cognitive function over time and across ages?

3. What are the adaptive responses to changes in physical, psychological and cognitive function over time and across ages?

4. How do changes in functioning, and adaptations determine successful ageing?

\subsubsection{Measures and Rationale}

The objective measures of physical health were selected to capture the respondents' health and functional status as fully as possible in a given time (approx $2 \frac{1}{2} 2-3$ hours).

The criteria used in selecting measures were:

a. High quality measures that can provide valid and reliable baseline data;

b. Proven measures from scientific research, plus measures that are novel to longitudinal research on ageing;

c. Instruments that are used in other national and international surveys in order to maximise comparability; and,

d. Minimising respondent burden. 
Table 9.1 below lists the measures that were selected by the working groups for inclusion in the TILDA Health Assessment. Standard Operating Procedures (SOPs) were developed for each test. These were based on validated procedures and adapted from international studies where possible and chosen to maximise comparability.

Where no suitable SOP was available, one was devised by the TILDA health team, in collaboration either with the suppliers of the equipment or with other research staff. The SOPs were then compiled into a training manual for the research nurses.

Table 9.1 TILDA Objective Health Measures

\begin{tabular}{|c|c|c|}
\hline Working Group & Measure & Rationale for Measurement \\
\hline \multirow[t]{2}{*}{ Neuropsychological } & $\begin{array}{l}\text { Mini Mental State Examination } \\
\text { Montreal Cognitive Assessment }\end{array}$ & $\begin{array}{l}\text { Rapid screening instruments for } \\
\text { assessment of global cognitive function. }\end{array}$ \\
\hline & $\begin{array}{l}\text { Sustained Attention* } \\
\text { Choice Reaction Time } \\
\text { Visual Memory } \\
\text { Executive Function }\end{array}$ & $\begin{array}{l}\text { By testing a range of cognitive abilities } \\
\text { and examining patterns of performance } \\
\text { in different cognitive areas TILDA can track } \\
\text { cognitive function and decline in different } \\
\text { brain areas over time. }\end{array}$ \\
\hline \multirow[t]{4}{*}{ Cardiovascular } & Waist-Hip Ratio & $\begin{array}{l}\text { There has been increasing interest in the } \\
\text { distribution of body fat as an important } \\
\text { indicator of increased risk of cardiovascular } \\
\text { disease. The waist-to-hip ratio is a measure } \\
\text { of distribution of body fat (both } \\
\text { subcutaneous and intra-abdominal). }\end{array}$ \\
\hline & Heart Rate Variability* & $\begin{array}{l}\text { Heart rate variability is a powerful } \\
\text { measure of autonomic nervous system } \\
\text { function. TILDA is looking at its association } \\
\text { with falls and cognitive impairment. }\end{array}$ \\
\hline & Pulse Wave Velocity* & $\begin{array}{l}\text { Pulse wave velocity is an indicator } \\
\text { of arterial stiffness and is a surrogate } \\
\text { marker for atherosclerosis. }\end{array}$ \\
\hline & Phasic Blood Pressure* & $\begin{array}{l}\text { Phasic blood pressure allows for the accurate } \\
\text { capture of dynamic blood pressure. It will } \\
\text { facilitate in-depth assessment of orthostatic } \\
\text { hypotension, a major risk factor for falls in } \\
\text { older people. }\end{array}$ \\
\hline \multirow[t]{2}{*}{ Gait, Balance \& Sensory } & Timed up \& Go & $\begin{array}{l}\text { Quantifies functional mobility and is useful } \\
\text { in following clinical change over time. }\end{array}$ \\
\hline & Gait Assessment* & $\begin{array}{l}\text { TILDA aims to investigate changes that } \\
\text { occur in gait patterns as people age using } \\
\text { a sensored mat. Reliable predictors of fall- } \\
\text { prone elderly can then be developed and } \\
\text { used to develop a programme for } \\
\text { preventing falls. }\end{array}$ \\
\hline
\end{tabular}




\begin{tabular}{|c|c|c|}
\hline \multirow[t]{3}{*}{ Working Group } & Measure & Rationale for Measurement \\
\hline & Balance & $\begin{array}{l}\text { Balance is needed to successfully carry out } \\
\text { every day locomotor functions at reasonable } \\
\text { speeds and to prevent falls. }\end{array}$ \\
\hline & Visual acuity \& contrast sensitivity & $\begin{array}{l}\text { Visual impairment is strongly associated with } \\
\text { physical disability among older adults and is } \\
\text { a significant risk factor for balance problems } \\
\text { and falls. }\end{array}$ \\
\hline \multirow[t]{2}{*}{$\begin{array}{l}\text { Strenght and } \\
\text { Bone Density }\end{array}$} & Grip Strength & $\begin{array}{l}\text { Grip strength is a powerful predictor } \\
\text { of disability, morbidity and mortality } \\
\text { and is a useful marker of frailty. }\end{array}$ \\
\hline & Heel Ultrasound & $\begin{array}{l}\text { A heel ultrasound measures the speed of } \\
\text { sound through a bone (the heel) and is used } \\
\text { as a surrogate marker for osteoporosis. }\end{array}$ \\
\hline \multirow[t]{2}{*}{ Macular degeneration } & Macular Pigment Optical Density* & $\begin{array}{l}\text { Age-related macular degeneration (AMD) } \\
\text { is the leading cause of irreversible blindness } \\
\text { in people over } 50 \text { years of age, affecting an } \\
\text { estimated } 80,000 \text { people in Ireland } \\
\text { alone. TILDA is measuring macular pigment } \\
\text { to investigate its potential role in preventing } \\
\text { age-related macular degeneration (AMD). }\end{array}$ \\
\hline & Retinal Photograph* & $\begin{array}{l}\text { Retinal images will be evaluated to } \\
\text { characterise and grade the extent and } \\
\text { severity of macular degeneration, if any, } \\
\text { according to standardised protocols. }\end{array}$ \\
\hline
\end{tabular}

* novel areas of research in longitudinal studies of ageing

\subsubsection{Data Collection Model}

Table 9.2 illustrates the models used to collect objective measures of physical health in some other longitudinal studies of ageing. Since many of the biomeasures selected for use in TILDA are novel and involve complex technologies, it was agreed that fixed health assessment centres would be the most appropriate model for TILDA. 
Table 9.2 Models of data collection

\begin{tabular}{|l|c|c|c|}
\hline Study & $\begin{array}{l}\text { Health Measures taken } \\
\text { by Lay Interviewers }\end{array}$ & $\begin{array}{l}\text { Nurse Home } \\
\text { Assessment }\end{array}$ & $\begin{array}{l}\text { Centre based } \\
\text { Assessment }\end{array}$ \\
\hline $\begin{array}{l}\text { English Longitudinal } \\
\text { Study on Ageing (ELSA) }\end{array}$ & $\checkmark$ & \\
\hline $\begin{array}{l}\text { Survey of Health and } \\
\text { Retirement in Europe (SHARE) }\end{array}$ & $\checkmark$ & & \\
\hline Health \& Retirement Study (HRS) & $\checkmark$ & $\checkmark$ & \\
\hline $\begin{array}{l}\text { Amsterdam Study of the Elderly } \\
\text { Rotterdam Study }\end{array}$ & & & $\checkmark \times 2$ visits \\
\hline $\begin{array}{l}\text { National Social Life, } \\
\text { Health \& Aging Project }\end{array}$ & $\checkmark$ & $\checkmark \times 2$ visits & \\
\hline $\begin{array}{l}\text { Newcastle 85+ Study } \\
\end{array}$ & & \\
\hline
\end{tabular}

\subsubsection{Consent}

Ethical approval for the health assessment was provided by the Faculty of Health Sciences Research Ethics Committee at Trinity College Dublin. Respondents who agree to a health assessment are required to provide separate written consent for each of the following:
a. Physical assessment
b. The physical assessment and blood results to be forwarded to their General Practitioner or nominated doctor
c. The blood results to be forwarded to the respondent's own home
d. Donation of blood samples for immediate analysis
e. Storage of blood for future medical research
f. Extraction and storage of DNA from the blood samples

The respondent is provided with copies of the signed consent forms. 


\subsection{Pilot Outcomes}

\subsubsection{Need for a Home Health Assessment}

A major outcome from the analysis of the pilot data was the necessity for a home health assessment to capture data on participants who were unable or unwilling to attend the health assessment centre. Respondents who attended a health centre in pilot 1 tended to be in better health than the average for the study with high levels of cognitive ability and physical function. This suggested a potential bias towards healthy individuals attending the health centres. To further assess this potential bias, the option of a home health assessment was included in pilot 2 for those individuals who were unable or unwilling to attend a health centre.

Table 9.3 compares the demographics and self-reported health status of both the centre and home populations for pilot 2. It is clear that the home populations are very different to the populations attending the health centres. They are older, with lower levels of education and self-reported physical and emotional health. A model of data collection that included inhome as well as centre based physical assessments was therefore selected for TILDA to avoid systematically over-representing younger, healthier respondents.

Table 9.3 Comparison of Total, Centre-Participating and Home-Participating Populations in TILDA pilot 2

\begin{tabular}{|c|c|c|c|c|}
\hline N (\%) & & CAPI, N=291 & Centre, $\mathrm{N}=138$ & Home, $\mathrm{N}=38$ \\
\hline Age (mean, se) & & $63.7,9.8$ & $62.3,8.7$ & $70.1,10.1$ \\
\hline Sex & $\begin{array}{l}\text { Male } \\
\text { Female }\end{array}$ & $\begin{array}{l}131(45) \\
160(55)\end{array}$ & $\begin{array}{l}63(45.7) \\
75(54.4)\end{array}$ & $\begin{array}{l}17(44.7) \\
21(55.3)\end{array}$ \\
\hline Health insurance & $\begin{array}{l}\text { Full medical card } \\
\text { GP visit card } \\
\text { Private } \\
\text { None }\end{array}$ & $\begin{array}{c}133(45.7) \\
9(3.1) \\
165(56.7) \\
33(11.3)\end{array}$ & $\begin{array}{c}44(31.9) \\
2(1.5) \\
101(73.2) \\
20(14.5)\end{array}$ & $\begin{aligned} & 32(84.2) \\
& 1(2.6) \\
& 14(36.8) \\
& 0(0)\end{aligned}$ \\
\hline Marital status & $\begin{array}{l}\text { Married } \\
\text { Single } \\
\text { Separated } \\
\text { Divorced } \\
\text { Widowed }\end{array}$ & $\begin{array}{c}181(62.4) \\
36(12.4) \\
12(4.1) \\
13(4.5) \\
48(16.6)\end{array}$ & $\begin{array}{c}105(76.6) \\
14(10.2) \\
3(2.2) \\
6(4.4) \\
9(6.6)\end{array}$ & $\begin{array}{c}17(44.7) \\
5(13.2) \\
2(5.3) \\
0(0) \\
14(36.8)\end{array}$ \\
\hline $\begin{array}{l}\text { Highest educational } \\
\text { attendance }\end{array}$ & $\begin{array}{l}\text { University/college } \\
\text { Secondary school } \\
\text { Primary school }\end{array}$ & $\begin{array}{l}76(26.1) \\
130(44.7) \\
85(29.2)\end{array}$ & $\begin{array}{l}55(39.9) \\
63(45.7) \\
20(14.5)\end{array}$ & $\begin{array}{c}2(5.3) \\
10(26.3) \\
26(68.4)\end{array}$ \\
\hline Self-rated health & $\begin{array}{l}\text { Excellent } \\
\text { Very good } \\
\text { Good } \\
\text { Fair } \\
\text { Poor }\end{array}$ & $\begin{array}{l}50(17.2) \\
82(28.2) \\
87(29.9) \\
47(16.2) \\
25(8.6)\end{array}$ & $\begin{array}{c}30(21.7) \\
42(30.4) \\
41(29.7) \\
20(14.5) \\
5(3.6)\end{array}$ & $\begin{array}{c}3(7.9) \\
4(10.5) \\
12(31.6) \\
7(18.4) \\
12(31.6)\end{array}$ \\
\hline Self-rated mental health & $\begin{array}{l}\text { Excellent } \\
\text { Very good } \\
\text { Good } \\
\text { Fair } \\
\text { Poor }\end{array}$ & $\begin{array}{c}75(25.8) \\
111(38.1) \\
77(26.4) \\
24(8.3) \\
4(1.4)\end{array}$ & $\begin{array}{c}42(30.4) \\
51(36.9) \\
35(25.4) \\
7(5.0) \\
3(2.1)\end{array}$ & $\begin{array}{c}6(15.8) \\
12(31.6) \\
13(34.2) \\
7(18.4) \\
0\end{array}$ \\
\hline
\end{tabular}




\subsubsection{Timings}

The average time to complete the assessment in pilot 2 was 2 hours 45 minutes (range: 1 hour \& 20 minutes to 4 hours \& 15 minutes).

\subsubsection{Assessing Respondent Burden}

All respondents who completed a health assessment were asked to complete an evaluation form. Ninety nine percent of respondents rated the health assessment as either excellent or very good. Regarding the length of the assessment, 47 respondents (66\%) found the length "just right", $23(32 \%)$ "a little long" and 1 (2\%) "very long".

On the whole, respondents were satisfied with the amount of feedback they received after they had completed the health assessment. However, respondents in Pilot 1 indicated their disappointment at not receiving feedback on their cholesterol levels if they had given a blood sample. It was therefore decided to include this in Pilot 2 of the main wave of TILDA and to post respondents their full lipid profile with a general explanation of those results within two weeks of attending for a health assessment in Wave 1 of TILDA.

\subsubsection{Amendments to Individual Tests}

\section{Hearing}

The majority of participants found the audiogram test included in the first TILDA pilot difficult. In particular, the noise-excluding headphones were felt to be too heavy, especially amongst older respondents. After consultation with the Working Group, a decision was taken to exclude the audiogram from the health assessment. Respondents currently self-report hearing loss in the CAPI interview and research has shown that this is sufficiently sensitive and specific to provide reasonable estimates of hearing loss prevalence (Valete-Rosalino and Rozenfield, 2005).

\section{Contrast Sensitivity}

The Pelli-Robson chart was used to assess contrast sensitivity in pilot 1 and 2 . This chart has been designed to be administered in a bright room without glare. Although attempts were made to standardise illumination levels in all five rooms where contrast sensitivity is assessed, the effect of environmental variables could not be completely eliminated.

After consultation with experts in the National Optometry Centre, the method of assessing contrast sensitivity was changed to a computer-based test called the "Functional Vision Analyser". As this machine is internally illuminated, it is not influenced by differing light levels within examination rooms. It also facilitates the testing of contrast sensitivity at precisely controlled photopic (day) and mesopic (night) conditions, both with and without glare, in accordance with the current established research standards. 


\subsubsection{Medical Card Number}

Medical Cards are issued by the Department of Health (through the Health Service Executive) and are means-tested. A person with a medical card is able to receive certain health services (such as access to a GP, prescription medicines and hospital care) free of charge. The medical card number (GMS number) is useful for tracking health service utilisation within the public health service as well as allowing for the possibility of linking TILDA data with the GMS prescription medication database.

In pilot 1, respondents who attended a health assessment were asked if they would provide the TILDA study with their medical card number if requested to do so. Seventy five percent of respondents (see Table 9.4) said they would have been happy, if asked, to provide this data so that TILDA could link their information with official health records.

Table 9.4 Respondents' opinion on providing TILDA with GMS number (Pilot 1)

\begin{tabular}{|l|c|c|c|}
\hline Question & $\begin{array}{c}\text { Yes } \\
\text { N (\%) }\end{array}$ & $\begin{array}{c}\text { No } \\
\text { N (\%) }\end{array}$ & $\begin{array}{c}\text { No Opinion } \\
\text { N (\%) }\end{array}$ \\
\hline $\begin{array}{l}\text { "Some studies like TILDA link the information they } \\
\text { collect with official health records to provide a } \\
\text { complete picture about the health and treatment } \\
\text { history of the participant. If we had asked you to } \\
\text { provide us with your medical card number for this } \\
\text { purpose would you have been happy to do so?" }\end{array}$ & $49(75 \%)$ & $6(10 \%)$ & $10(15 \%)$ \\
\hline
\end{tabular}

All respondents in pilot 2 were asked to provide their medical card number during the CAPI interview, with 147 respondents (68\%) providing this information. As a result of both pilots, all respondents are now asked to provide their GMS number during the social interview.

\subsubsection{Consent for Stored Blood Sample and DNA Extraction}

All respondents who had a health assessment in pilot 2 were asked about their willingness to allow TILDA to store some of their blood and conduct and store a DNA extraction on their blood. Specifically, they were asked if they would have provided consent, if they had been requested to do so, for the following:

a.Samples of their blood to be stored and used in future medical research studies.

b.The extraction and storage of DNA from blood samples they had provided.

168 respondents (95\%) indicated that, if asked, they would be willing to provide consent for a stored sample while 166 respondents (94\%) would be agreeable to providing consent for genetic analysis. Based on this positive feedback, respondents in wave 1 of the TILDA study are asked to give separate consent for storage of samples for future analysis and for the extraction and storage of DNA. 


\subsection{Health Assessments}

All respondents who complete the main wave 1 interview are eligible for a health assessment. The health centre assessment is introduced to the respondent first. In the event that the respondent is unable or unwilling to attend a health centre, the social interviewer introduces the home assessment (see Figure 9.1 below).

The health assessments, both in the centre and in the home, are carried out by a team of specially trained and qualified nurses who work under the supervision of the TILDA Nurse Manager and the TILDA Health Assessment Manager.

\subsubsection{Setting up a Health Assessment Appointment}

The process for setting up either a health centre assessment or a home assessment is similar and is outlined in Figure 9.1. If a respondent refuses a health assessment the reason for refusal is recorded in CAPI. All travel expenses relating to the health centre assessment are reimbursed by the TILDA study on the day of the health assessment, to the extent possible.

\section{Figure 9.1 The process for setting up a health assessment appointment}

The social interviewer provides information about the health assessment and invites the respondent to attend the health assessment centre

If the respondent agrees, the social interviewer telephones the TILDA Health Executive Officer who arranges a date and time for the health assessment which must be at least 1 week from the date of the social interview. (Out of office hours, the interviewer leaves a message containing the respondent's name, phone number \& TILDA ID and the Executive Officer returns the call the next day to arrange an appointment).

If an appointment is made during the social interview, an appointment card is left with the respondent and s/he is given a Health Centre Information Booklet.

If the respondent is not willing to attend a health assessment centre, the social interviewer provides information about a home health assessment and offers the respondent a home assessment by a trained nurse.

If the respondent agrees to a home assessment, the social interviewer phones the Health Executive Officer and informs her that a respondent has agreed to a home assessment. The social interviewer informs the respondent that a nurse will telephone them to arrange an appointment sometime during the next few weeks. The respondent is left a Home Health Assessment Information Booklet. The Executive Officer compiles a list of all respondents who have agreed to a home assessment and forwards the details to the TILDA Nurse Manager. The Nurse Manager in turn allocates the home assessments to a specific nurse who telephones the respondent to set up an appointment for a home health assessment.

If the respondent is unsure or requires time to decide, the interviewer requests permission for a member of the TILDA team to phone the respondent in the next week to answer any questions and attempt to arrange either a health centre or a home assessment appointment. 


\subsubsection{Measures}

\section{Health Centre:}

The measures were discussed in Table 9.1 earlier in this chapter. A complete list of measures conducted at the health centres is detailed in Appendix 5, along with the number of readings taken and the type of / details of the equipment used.

\section{Home:}

The measurements conducted in the home assessment are a subset of the measures conducted in the health centre and are detailed in Appendix 6.

\subsubsection{Order of the Tests}

\section{Health Centre:}

The health centres have been designed to allow nurses to assess three respondents at any one time. Each centre is divided into 4 different stations which correspond to one or more test types:

- Station 1 - 3*: Cognitive \& Core Measures

- Station 4: Vision

- Station 5: Gait \& Balance

- Station 6: Cardiovascular

Figure 9.2 Flow of respondents through the TILDA Health Assessment Centre

\begin{tabular}{|c|c|c|c|}
\hline & 1st respondent & 2nd respondent & 3rd respondent \\
\hline Start & $1 *$ & $2^{*}$ & $3^{*}$ \\
& 4 & 5 & 6 \\
Finish & 5 & 6 & 4 \\
\hline
\end{tabular}

* Station 1, 2 and 3 are all equipped the same.

All respondents start with the cognitive function station (station 1-3). Within this station, all cognitive tests are completed in a fixed order. Respondents can then complete the other stations in a number of predetermined sequences (Figure 9.2). The placement of station 1 as the start point for all participants was designed to avoid the problem of fatigue potentially influencing performance in the cognitive tests. In addition we wanted to ensure that respondents did not go from Station 6 directly to Station 5, because a postural drop in BP could potentially influence the outcome of gait \& balance tests.

\section{Home:}

All respondents assessed at home complete the cognitive tests first (in the same fixed order as in the centre assessments) before the other physical measures are taken. 


\subsubsection{Data Collection}

\section{Health Centre:}

TILDA specific software has been developed by the Trinity Centre for High Performance Computing to electronically record all data collected in the health centres and to transfer the data to a secure database. Briefly, the data collection process proceeds as follows:

a. Once a respondent arrives at the health centre, the nurse formats an encrypted USB key with the respondent's unique TILDA ID.

b. As the nurse moves from station to station, the results are uploaded from each station onto the USB key.

c. Once the assessment is complete, the data is uploaded securely to a firewalled server in Trinity College Dublin.

d. The nurse then deletes the data, for that individual, from the USB key.

\section{Home:}

TILDA specific software was developed with an external contractor to electronically capture all data collected in the homes. Briefly, the data collection process is as follows:

a. The nurse enters all data onto a hand held personal digital assistant (PDA) (see Fig 9.3).

b. Once the assessment is complete, the encrypted data is transported by General Packet Radio Services (GPRS) to the contractor's secure server.

c. Data is compiled into Excel spreadsheets for secure transfer to TILDA from the contractor's server at regular intervals.

Figure 9.3 An image of the PDA used to collect home health assessment data

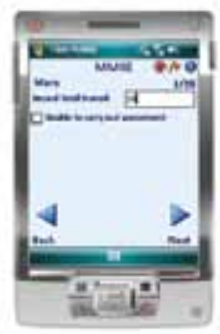

\subsubsection{Quality Assurance}

\section{Initial Training}

A comprehensive training programme was developed to ensure that all registered nurses employed to carry out health assessments were both competent and confident prior to the start of wave 1. Nurses were introduced to the concepts of survey research focusing on the specific skills required for an observational study like TILDA. Nurse protocols were developed for all measures and the nurses were trained in accordance with these. Health centre nurses received a total of 4 weeks training and completed up to 50 assessments on volunteers and pilot participants prior to wave 1 . Nurses employed to carry out the home assessments received 
one week of training and completed a minimum of 6 assessments on volunteers prior to deployment to respondents' homes.

\section{Continuing Education/Training}

All nurses must attend quality control and study days scheduled every 6-8 weeks after initial training, for further training pertinent to their roles as research nurses.

\subsubsection{Quality Control}

Quality control (QC) is a key priority for the TILDA health team. The following steps have been implemented to ensure all data collected is of the highest standards:

a. Supervision - both health centre and home nurses are shadowed during several assessments by a member of the TILDA health team to ensure all measures are administered as per the protocol. This shadowing is front loaded in that it occurs more frequently at the start of the project in order to troubleshoot and correct any problems early on.

b. Frequent Quality Control / Study Days - all nurses are required to attend these meetings which occur every 6 - 8 weeks after initial training. The objective of these days is two-fold - first to trouble shoot and disseminate solutions about any issues that may have arisen in the past 6 - 8 weeks, with regard to the measurements, the QC procedure or the study itself. Second, these days incorporate teaching and further training so that the nurses can expand their knowledge base.

c. Validation - both software systems have been developed with specific ranges for each measurement to ensure only "reasonable" values can be entered into the computer for each test. For example, the computer will not accept a MMSE score of 35 as the maximum score for this test is 30 .

d. All MMSE and MOCA cognitive tests are re-scored by a member of the TILDA health team. If an error is found, the correct test score is indicated on the paper test and is scanned with the correction and signature of the TILDA health team member making the correction, as an audit trail of that change. In addition, the correct score is substituted into the database, at which point the software creates a full audit trail of any changes.

e. All equipment is calibrated as per manufacturer's protocols. TILDA also has on-call and/or onsite support for equipment issues provided through collaboration with St. James's Hospital Medical Physics and Bioengineering Department.

\subsubsection{Respondent Feedback}

At the end of the assessment, respondents are given a feedback sheet (see Appendices 7-8) with the following results:

- Health Centre assessment: height, weight, blood pressure, heel ultrasound \& visual acuity.

- Home assessment: height, weight and blood pressure. 
Results are graded as normal or abnormal and the nurse responds to any queries relating to the results that a respondent has received. The nurses are trained to deal with abnormal results. If there is uncertainty or concern on the part of the nurses, they are instructed to contact the TILDA Health Assessment Manager to discuss the matter further.

If the respondent provides a blood sample, a full lipid profile is posted to the respondent's home within 2 weeks. A leaflet explaining the various types of cholesterol is included so the respondent can interpret the result. If the respondent provides consent, a copy of their blood results is also forwarded to their GP (General Practitioner). The blood feedback form and accompanying information about cholesterol are in Appendices 9-10.

\subsection{Blood Collection}

\subsubsection{General Approach to Sample Collection}

The strategy for the collection of biological samples in TILDA was led by a number of important principles. The aim was to collect samples that would facilitate the analyses of measures currently known to be of interest for describing and studying ageing while also allowing the widest possible range of assays that could plausibly be envisaged for the future.

We also wanted to avoid collection, processing or storage approaches that would inherently preclude such assays (i.e. "future proof" the collection as far as possible given current knowledge and available resources). As the sample collection occurs in both fixed centres and homes, the use of processes that would allow identical processes in both settings was considered a high priority. The TILDA blood sampling procedures are the result of extensive consultation and peer review in the scientific community in Ireland, the UK and the US, followed by extensive piloting to ensure that the proposed procedures were fit for purpose. TILDA-specific SOPs for blood collection and processing were written to detail the samples to be collected, the transport of samples to a central laboratory, and the processing, aliquoting and storage of each sample.

\subsubsection{Biological Samples to be Collected}

In addition to the members of the TILDA Biomeasures Working Group (see Appendix 4), TILDA also consulted with Dr. Kamna Das from the Columbia University Medical Center in New York, specifically with regard to the genetic analysis. One remit of the Biomeasure group was to determine the likely value of the additional information that would become available by collecting a particular sample type (i.e. the range of assays that could be made and the physiological coverage of the material on plasma, serum, or whole blood samples). This was constrained by the maximum amount of blood that could be collected, namely $25 \mathrm{ml}$, proposed by TILDA for the baseline assessment visit (based on common practice in other, similar studies) 
and approved by the Ethics Committee. One agreed outcome was to dedicate some of the sample for future genetic analysis, particularly as this type of sample (buffy coats) is robust and requires a small volume of blood. Furthermore, the feasibility and cost of collecting and processing such samples was taken into consideration, particularly as some of the assessments would occur in respondents' homes.

The collection of other biological samples including urine, nails and saliva was also considered (Table 9.5). These other methods of collection were ultimately excluded as it was decided that these methods did not provide sufficient additional information to justify the additional cost or complexity required.

Table 9.5 Excluded Methods of Biological Sampling

\begin{tabular}{|l|l|}
\hline Sample type & Exclusion criteria \\
\hline Nails & $\begin{array}{l}\text { Limited additional information } \\
\text { (e.g. exposure to environmental heavy metals). } \\
\text { Complicating effects of cosmetic products. } \\
\text { Inconsistency of sample collection. } \\
\text { Possible impact on recruitment of clipping nails. }\end{array}$ \\
\hline Saliva & $\begin{array}{l}\text { Limited additional information (e.g. indicators } \\
\text { of periodontal disease and oral cancer). } \\
\text { Extra cost of storage. }\end{array}$ \\
\hline Urine & $\begin{array}{l}\text { Difficulty of collection. } \\
\text { Extra cost of storage. }\end{array}$ \\
\hline
\end{tabular}

\subsubsection{Types of Sample Collection Tubes \& Supplies}

There is a very wide variety of preservatives and additives available for the collection of blood. The selection of the preservatives and additives is important in determining the future applicability of the samples. The preservatives considered for TILDA included EDTA and Lithium Heparin plasma, Acid Citrate Dextrose (ACD), Paxgene and Silica.

Collection tubes coated with EDTA provide blood fractions suitable for a wide range of DNAbased and protein assays and these tubes can also be used for routine biochemistry. Lithium heparin tubes are preferred for metabonomic studies and are used by commercial companies for the measurement of lipids. Tubes containing ACD are required for peripheral blood lymphocytes to produce immortalized cell lines. The high cost of the Paxgene tubes prohibited their inclusion in this wave of the study. The requirement for storage at $18^{\circ} \mathrm{C}$ for the $A C D$ tube, combined with the fact that DNA could be available from other tubes (e.g. EDTA plasma), resulted in the exclusion of this tube from the final model. As RNA is particularly sensitive to degradation by abundant and ubiquitous RNAases, the use of tubes with RNAase inhibitors 
is required to maintain RNA integrity. While these tubes are available commercially, they are expensive. Lastly, small plastic tubes containing silica are available and can be used to collect serum.

Inevitably, the selection of additives is a compromise, and the final decision for TILDA, of using a combination of EDTA and Lithium Heparin tubes, was made in order to cover as wide a range of current and potential future uses as is feasible given the study design and available resources.

The inclusion of both centre and home based health assessments is considered essential to minimise selection bias in the biological sampling component of TILDA. High priority was given to ensuring that identical processes were undertaken for bloods collected in the home and centres.

TILDA initially identified guidelines for blood sample handling that stated that plasma or serum should be separated from cells as soon as possible and certainly within 2 hours (Young and Bermes, 1999). This requirement would have resulted in the need for either immediate local separation of blood samples or their rapid-chilled transfer to a central laboratory, which would have increased complexity and cost. The perceived need for such processing had initially precluded blood collection for long-term storage from the TILDA home respondents as it required local processing facilities that were not available. As a result, this was not considered an appropriate approach.

However, following advice from national and international experts, as well as consultation with the emerging literature, it became clear that a wide range of analytes are stable in plasma (but not in serum) for periods up to 48 hours (Clark et al., 2003, Clark et al., 2004, Drammeh et al., 2008). As a consequence, it was decided to process and separate plasma, and the buffy coats needed for subsequent genetic analysis, from all TILDA blood samples.

Each set of required tubes has a unique bar-code that is scanned into the assessment centre IT system or in the home into the nurse's handheld scanner to link each tube with the participant's unique TILDA ID. A collection priority is specified in the event that the assessment nurse is unable to extract sufficient blood for the full set of tubes (Table 9.6). All tubes are transported to the central processing laboratory in temperature-controlled shipping boxes which maintain the samples at $2-8^{\circ} \mathrm{C}$ for up to 48 hours.

Table 9.6 Sample Collection Priority, Volume and Transport Temperature

\begin{tabular}{|l|c|c|c|}
\hline Type of Sample & Collection Priority & Volume Collected (ml) & Temperature \\
\hline Lithium heparin & 1 & 5 & $4{ }^{\circ} \mathrm{C}$ \\
\hline EDTA & 2 & 10 & $4{ }^{\circ} \mathrm{C}$ \\
\hline EDTA & 3 & 10 & $4{ }^{\circ} \mathrm{C}$ \\
\hline
\end{tabular}




\subsubsection{Transport and Central Processing Methodology}

On an average day, TILDA nurses assess a total of 12-18 participants in the two assessment centres, (Dublin and Cork) as well as 4-5 in participants' homes. Since each sample consists of three blood vials, this yields an average of 66 separate tubes per day, which are transported to the central laboratory for further processing.

TILDA chose a central laboratory for blood work to facilitate storage in one facility and batch processing of bloods. Following an evaluation of packaging options and courier services in Ireland, conducted by ADP Pathology Storage Ltd., the TILDA blood services supplier, TILDA chose to invest in the Biotherm 5 system of temperature-controlled boxes for the transportation of biological samples. These conform to all United Nations 3373 regulations for such transport. Furthermore, TILDA chose to work with DX, a company specializing in pickup and delivery of confidential material within short timeframes throughout Ireland.

The DX courier collects samples from each TILDA centre on a daily basis (Monday-Saturday). The home nurses use a network of thirty eight drop off points located strategically throughout the country. The blood boxes are left in secure areas within the drop off points and are collected daily (Monday - Friday) by the courier. Special arrangements are in place for collection of home blood samples on a Saturday.

The aim is to ensure that all samples are transported to the laboratory and processed within 48 hours. As indicated in Table 9.7, the different samples from each individual yield up to 10 aliquots of $1 \mathrm{ml}$ volume for long-term frozen storage. The rationale for storing this number of separate aliquots for each individual is to provide sufficient amounts of each type of sample for a wide range of end-of-wave and future analyses during long-term follow-up and to protect the samples from repeated rounds of freezing and thawing, which is not an optimal long-term practice as it may affect the validity of results and requires more complicated tracking.

Table 9.7 Fractions and Aliquots of Blood Samples

\begin{tabular}{|c|l|c|}
\hline Vacutainer Tube & Fractions & No of Aliquots \\
\hline EDTA $(10 \mathrm{ml} \times 2)$ & Plasma & 7 \\
& Buffy Coat & 2 \\
\hline Lithium Heparin & Immediate & N/A \\
& Plasma & 1 \\
\hline
\end{tabular}


Only those assays (lipid profile) that are required immediately (to provide feedback to participants) are performed as samples arrive at the central laboratory in order to streamline processing, improve cost-effectiveness and to minimise quality control issues. Other measures conducted in wave 1 will be done at the end of the wave in a batch process.

Although TILDA's current storage is in $-80^{\circ} \mathrm{C}$ freezers, TILDA has decided to store its frozen blood samples in cryovials in order to facilitate the potential long-term storage of the samples in liquid nitrogen. Liquid nitrogen storage is a recommended option for storage longer than 10 years. The use of cryovials at the start will eliminate the difficult and costly task of thawing blood samples to transfer from standard aliquot tubes into cryovials, should the study decide to do so in the future.

\subsubsection{Immediate and End-of-Wave Analyses}

As indicated earlier, pilot respondents reported wanting their cholesterol results and so TILDA decided that providing these results would be a good way to encourage participants to agree to the study and its health components, as well as a way to improve participants' long-term commitment to the study. As TILDA is a cohort study with repeated waves of data collection, it is of critical importance to include components that encourage adherence. Therefore cholesterol is measured immediately for all respondents who give a blood sample.

By the end of the first wave of data collection, TILDA will be storing about 60,000 aliquot tubes. It is envisaged a range of analyses will be undertaken on the complete sample at the end of the data collection period. One known measure that will be done by collaborators at the Waterford Institute of Technology at the end of wave 1 is carotenoid analysis which complements the measurements of macular pigment obtained in the health centre assessments. The remainder of the set of core bloods measures that TILDA will run at the end of the first wave has yet to be finalised. A proposed set of measures is under concurrent review and consideration by the TILDA Data Sharing subcommittee of the Steering Committee and the Scientific Advisory Board of international advisors. The decision will take into account key TILDA scientific areas. One guideline is to prioritise measures of diseases of known high importance in older age while also allowing for the development of potential novel biomarkers.

\subsubsection{Long-term Sample Storage}

Following these end-of-wave analyses, TILDA will seek to put stored samples from each participant in two geographically separate locations in order to protect the resource from loss. One location will house the "working" archive that will typically be used first for any research project and the other location will house the "back-up" archive that will be used when samples in the working archive have been exhausted. 

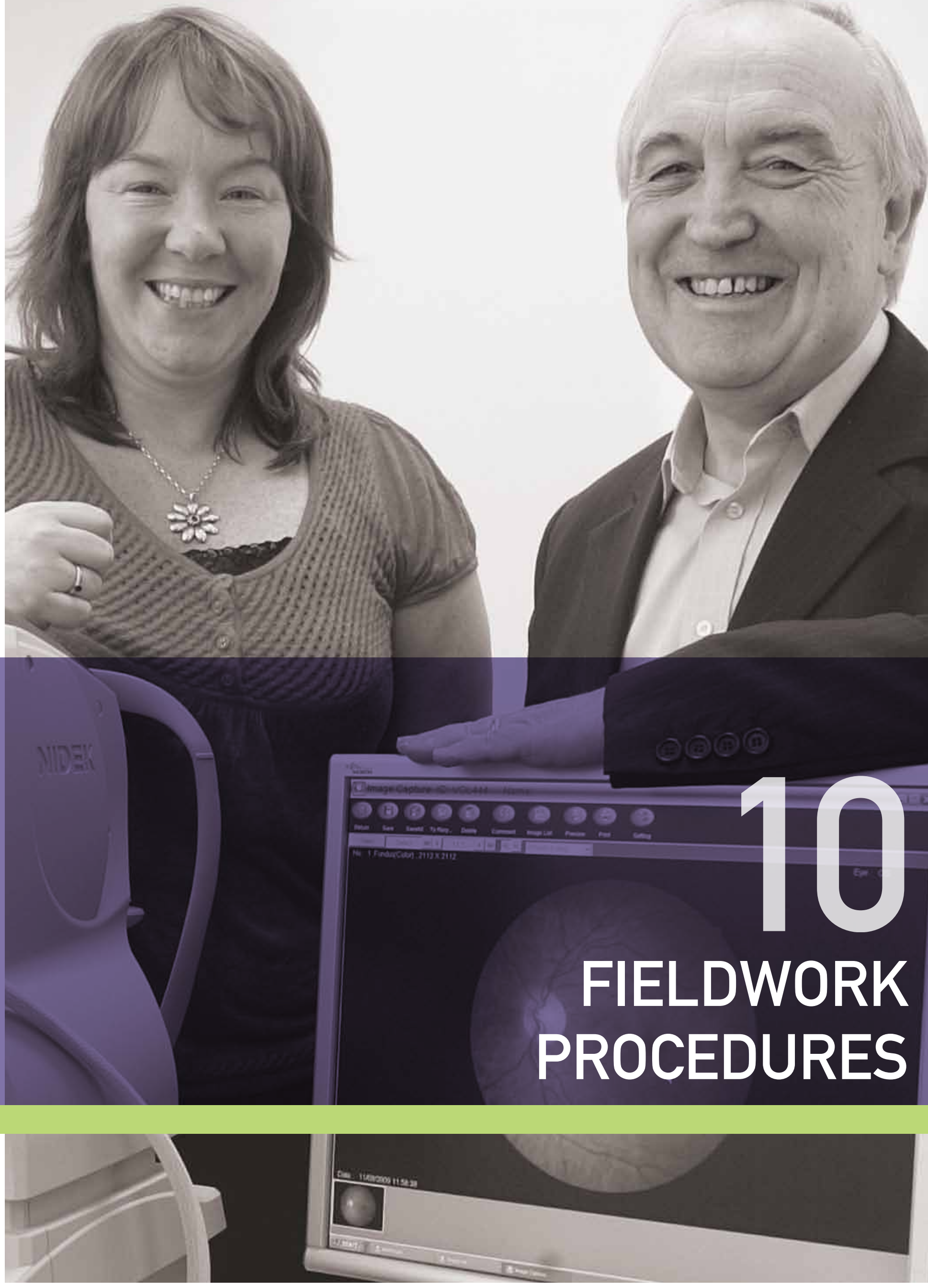


\section{fieldwork procedures}

\section{Introduction}

As described in earlier chapters, TILDA's data is collected using three modalities: the Computer-Aided Personal Interview (CAPI); the Self-Completion Questionnaire; and the Health Assessment, conducted either in a dedicated TILDA Centre, or in the respondent's home. To be included in the TILDA sample, all respondents must complete a CAPI interview. However, while respondents are strongly encouraged to fill in and return a Self-Completion Questionnaire and participate in a health assessment, these are not required elements of participation.

This chapter describes the fieldwork procedures undertaken for each type of modality (CAPI, Self-Completion Questionnaires and Health Assessments).

\subsection{CAPI Fieldwork}

\subsubsection{Pilot 1}

The first TILDA pilot was conducted in the Dublin area between the end of July and beginning of October 2008. It was based on a random selection of addresses generated by the ESRI's RANSAM system. The pilot was successful in many ways, such as confirming that interviewers could conduct the CAPI as scripted and, more importantly, that respondents were willing to answer the majority of interview questions. It also confirmed that the respondent burden was appropriate (less than 90 minutes duration) and in accordance with TILDA's Ethical approval.

The TILDA team provided a 3-day briefing for 11 interviewers selected to work on the first pilot, of which 8 ultimately conducted fieldwork. Since many of these interviewers did not have good computer skills, quite a lengthy amount of time was spent providing them with basic technical skills to improve their confidence using the laptops for CAPI.

Three main challenges arose from the first pilot for further consideration:

- first, the CAPI tool was not as robust as it needed to be in terms of design and support;

- $\quad$ second, the long respondent identifier proved too difficult to be suitable; and,

- $\quad$ third, the response rate was lower than hoped. 


\subsubsection{CAPI}

Since the TILDA questionnaire is lengthy, complicated and embodies extensive routing, (i.e. skips from one point to another depending on the answer to previous questions), it was decided from the outset to use a Computer-Aided Personal Interviewing (CAPI) system. The CAPI questionnaire for the first pilot was designed in MMIC, a programming language created by the RAND Corporation in California. It offered flexibility of design, in that its routing capabilities were good and it was designed to accommodate the distinction between individual and household data. However, since it was being managed and supported from California, it proved extremely difficult to make updates to the CAPI questionnaire itself and to deal with technical questions from the field while fieldwork was in progress. Furthermore, there was no sample management system incorporated into the TILDA CAPI at this time and so tracking fieldwork progress was an onerous, manual task. One of the main lessons from this pilot was the need to provide adequate technical training and assistance to interviewers both prior to and during the fieldwork period.

\subsubsection{The Respondent Identifier}

Although other longitudinal studies have used respondent identifiers as long as 15 digits, the experience with the TILDA study was that this would not be suitable for two reasons. First, some equipment in the health assessment centre could only take an identifier with a maximum of 5 characters, so for respondents to participate in those tests, it would be necessary to create a secondary identifier, which was not deemed a suitable long-term option for the study. Second, where interviewers needed to copy the number, i.e. onto the cover of the SelfCompletion Questionnaire, the long identifier led to more transcription errors, which then imposed a burden on staff to manually identify matching data for respondents from partial or incorrect identifiers. As a result, we decided to shorten the identifier going forward.

\subsubsection{Improving the Response Rate}

The initial sample in this pilot yielded a response rate of $41.8 \%$. A reserve sample was then launched to test the impact of improved interviewer training and fieldwork management practices. While this did raise the response rate to $46.7 \%$, this was still lower than anticipated. A number of factors were thought likely to have influenced the response rate. In the first place, pilot 1 was restricted to the Dublin area and surveys conducted in urban areas often suffer from lower response rates than in more rural areas. Second, the timing of the pilot during the summer months when people are on holiday may have had an effect. Interviewers had difficulty contacting members of selected households during this season and the short time period allowed for the first pilot was considered a constraint in that it did not allow interviewers to call back to addresses where they had failed to make contact; a longer fieldwork period was deemed a possible way to address this issue in the future. Third, the first pilot did not include any advertising or publicity to inform respondents about the study and encourage participation. As described below, a number of strategies were incorporated in pilot 2 to address these issues. 


\subsubsection{Pilot 2}

The second TILDA pilot was conducted throughout the Republic of Ireland between the beginning of April and the end of July 2009. The addresses were again generated by the ESRI's RANSAM system. This pilot was successful in testing the willingness of respondents across the country to respond to the CAPI, and to test their willingness to attend a dedicated TILDA Health Assessment Centre in Dublin or Cork.

One innovation in the second pilot was the introduction of satellite navigation (sat nav) devices. The ESRI supplied geographical co-ordinates for each address in the sample and Ipsos MORI, the contracted fieldwork agency, was able to program the sat nav's, with these addresses and coordinates, eliminating in almost all cases the need for interviewers to exercise discretion in identifying the selected dwelling within clusters or groups of adjacent houses. The sat nav's were well received by the interviewers and their use has been continued in the main wave.

As the interviewers in pilot 2 already had basic CAPI training prior to beginning on the TILDA project, two two-day briefing sessions were held in Dublin and Limerick to train a total of 26 interviewers and two regional coordinators. The briefings focused primarily on the CAPI content, identifying known areas of reluctance where respondents in pilot 1 had difficulty in answering, and suggesting hints or strategies for how to overcome reluctance and improve response.

\subsubsection{CAPI}

In response to the findings from the first pilot regarding the technical savvy needed to properly support the CAPI, TILDA sought to use locally based technical expertise for pilot 2 . This meant moving away from MMIC because local support and experience with this language is very limited. The CAPI for the second pilot was, therefore, programmed in Quancept by Ipsos MORI. This had a number of advantages:

(a) The interviewers were already trained and familiar with the language.

(b) The Ipsos MORI system incorporated a sample management system called E-progress, that was already in use as an add-on to the CAPI. This sample management system allowed for updates on fieldwork in an automated and systematic way so that managers, both at Ipsos MORI and within TILDA, could monitor success rates throughout the fieldwork period in real time.

(c) Ipsos MORI's familiarity and expertise with Quancept meant that the process of rebuilding and updating the CAPI was fairly streamlined. This also facilitated added functionality in the CAPI. For example, for one cognitive task, a countdown clock for 60 seconds that worked directly in the CAPI was built, eliminating the need for interviewers to carry and use stopwatches for this purpose. The CAPI was also programmed with audio files to 'read' word lists for other cognitive tasks rather than having these read out by the interviewers; this will improve the consistency of the tests and assure best results. 
Version control of the CAPI was maintained via a Word version of the complete document. Changes to the CAPI were tracked in different colours for each set of changes throughout the pilot's development and early implementation.

\subsubsection{The Respondent Identifier}

One of the main changes for the second pilot was the creation of a five character TILDA serial number, which identified each of the 30 clusters in the sample by a two-letter code, followed by a two-number identifier which identified each of the addresses in the cluster, followed by a one-number identifier for the individual respondent in the participating household. This addressed the concerns raised in pilot 1 about using secondary identifiers for respondents participating in health assessment measures where the equipment could only take five characters, and also improved the accuracy of the identifiers copied by interviewers onto the Self-Completion Questionnaire.

\subsubsection{Improving the Response Rate}

In order to address the response rate issue in the first pilot, TILDA introduced two new measures for the second pilot. First, TILDA created an advance letter that was posted to all of the addresses on the sample list. Advance letters were also posted directly and via interviewers to local Garda stations (police) to inform the Gardaí that interviewers were in the area and to confirm the bona fides of the study. In fact, TILDA received a number of calls verifying the legitimacy of the study and so these were deemed good additions to the fieldwork procedure. However, as nearly $40 \%$ of addresses in Ireland (primarily in the rural communities) are not unique (there is no national postcode system), many of the letters were returned as undeliverable. This led to a slight modification to this process for the first wave.

Second, respondent incentives were introduced to encourage participation. Since the literature in this area did not seem to advocate one consistent type or amount of incentive, TILDA opted to try two financial incentives - $€ 10$ and $€ 25$ - to see whether the increased amount or the availability of the increased amount would have any effect on response rate. Unfortunately, even with these incentives, the pilot response rate was only $45 \%$.

The completion of the second, national pilot raised two major questions for the first wave of TILDA regarding response rate. First, it highlighted the necessity for a publicity campaign to inform the public about the study and encourage participation; this was implemented for the first main wave. Second, TILDA decided to re-examine the respondent selection process. In the two pilots, interviewers had to select one eligible respondent in a household using a randomising rule (select the person aged 50 or over with the next birthday). Then, if that person agreed, interviewers were tasked with trying to interview that primary respondent's spouse or partner. In many instances, interviewers reported that they could have had participation from one eligible household member but were unable to do so because s/he was not the member selected using next birthday rule as the primary respondent. A smaller issue was raised regarding the timeframe. As in the first pilot, a longer fieldwork period was 
suggested so as to allow interviewers to achieve more interviews in households where they were unable to make contact, hopefully resulting in a higher response rate.

\subsubsection{Wave 1}

The first wave of TILDA data collection began in October 2009. All of the Ipsos MORI interviewers who had participated in the second TILDA pilot returned to work on the first wave. These interviewers received a one-day briefing that highlighted changes to the CAPI questionnaire since the second pilot and updates or reminders about particular fieldwork procedures. New interviewers were also recruited and trained for wave 1. Briefings for new interviewers occurred in 3-day sessions, where the objectives and nature of the study were described, the contact and sampling rules explained, the CAPI content was outlined and warnings, hints and tips about known areas where respondents had reluctance to participate were discussed. The interviewers participated in practice interviews to become familiar with running the CAPI in a simulated real-world situation. This 3-day format was an improvement over the 2-day format used in the second pilot. In total, 7 briefing sessions in both Dublin and Cork were held to train over 100 interviewers and several regional coordinators. Although Limerick had been used in the second pilot because of its easy accessibility for interviewers located to the west of the country, TILDA found that it was more effective to have interviewers visit and participate in a health assessment in order to more effectively encourage respondents to agree to one. As a result, trainings were held near the two TILDA dedicated Health Assessment Centres so that interviewers could visit the Centres.

\subsubsection{CAPI}

The ongoing collaboration of TILDA with Ipsos MORI for the main wave fieldwork meant the continued use of the Quancept-based CAPI. As in the second pilot, modifications to the questionnaire were tracked in a Word version, using different colours to indicate the different modifications carried out at different stages.

\subsubsection{Improving the Response Rate}

For the first wave of data collection, several changes were implemented to improve response rate. First, upon further consideration and reflection, it was decided that selecting one household member as the primary respondent and then his/her spouse or partner as a secondary respondent created two sets of difficulties:

(a) from a statistical point of view, it led to a bias in respondent selection since members of couples had a higher probability of selection than single individuals.

(b) from an operational point of view, response at household level was likely to be impaired because if the "primary" respondent was unwilling/unable to participate, this meant losing the whole household. 
Therefore, TILDA decided to allow all eligible members of a household to participate, including all people who were either him/herself aged 50 or over or the spouse or partner of someone aged 50 or over. This procedure, which is the method used in SHARE, also has the advantage of producing a sample which is self-weighting for both persons and households.

Second, TILDA chose to offer a $€ 20$ incentive to all participating respondents, based on the findings from the analysis of the incentives offered in the second pilot. Unlike in the second pilot, though, where interviewers disbursed cash, in the first wave, respondents are issued with a promissory note, which was redeemed by cheque by Ipsos MORI upon receipt of the CAPI data.

Third, several new fieldwork and management practices have been put in place to monitor progress throughout the time period. The advance letters to both Gardaí and selected addresses continues. However, for non-unique addresses, the advance letters and envelopes are given to interviewers directly rather than sending through the post. Interviewers working in those areas were instructed to post the letter once they arrive at the correct address.

Lastly, the major difference between the second pilot and the first wave of data collection is the use of public relations to improve the public's awareness of the study, thereby improving their willingness to participate and bolster response rate. The task was to identify and use methods of communication that would be effective to not only inform participants about the study, but also give them approval and encouragement to participate. This was done in several different ways.

- $\quad$ First, a radio ad with two known Irish celebrities, Olivia O'Leary and Micheál Ó Muircheartaigh, was made and was run during the first weeks of fieldwork on a variety of national radio stations. About four months into the fieldwork period, the ad was run again on a variety of local radio stations around the country.

- Second, an information brochure containing information about the project was sent to over 100 local church parishes, related community and non-governmental organisations, to be shared, posted and discussed at important community events.

- Third, TILDA team members participated in Over 50's shows in Dublin and Limerick.

- $\quad$ Fourth, TILDA Directors participated in interviews on both radio and television.

- $\quad$ Fifth, TILDA ran specific public relations events just prior to fieldwork commencing to create a buzz around the study. These included a public pensions forum, and a re-opening and launch of the public phase of the study by President Mary McAleese. These were very successful in informing the research community and public about the study and provided good opportunities for additional media coverage about the project. 


\subsection{Self-Completion Questionnaires}

The Self-Completion Questionnaire is paper-based and was printed in a bound booklet using a similar layout and design as other printed materials prepared for respondents. The Questionnaire, along with a stamped envelope addressed to the TILDA offices in Dublin, was left with participating respondents by the interviewer during an appointment for a CAPI interview.

In the first pilot, the greatest difficulty with these was helping interviewers write the correct and complete TILDA Serial number on the covers of these Questionnaires so that the data from them could be matched with data from other sources. As a result, we incorporated a prompt for interviewers in the CAPI to fill in the identifying information on the covers of the SelfCompletion Questionnaire. More specifically, near the start of the CAPI, the screen instructs the interviewer to put the specified TILDA Serial number for the participating respondent on the Self-Completion Questionnaire and to complete the cover of the Questionnaire, including the respondent's initials (in case we were unable to identify the respondent who completed a questionnaire from the TILDA Serial number), the interview date and the interviewer ID. In cases where both members of an eligible couple were participating, the CAPI prompted an interviewer to fill in the cover of a Self-Completion Questionnaire for the participating spouse/partner of a respondent first so as to facilitate completion of that questionnaire while the interview with the first respondent is undertaken. Interviewers in the second pilot reported that this was an effective tool to create some privacy during the CAPI interview. This has been maintained for the first wave.

Self-Completion Questionnaires are returned to TILDA via post. In many cases, interviewers are able to collect these and post them. Otherwise, respondents can post them back to TILDA themselves.

\subsection{Health Assessments}

While some of the fieldwork procedures and lessons learned from the pilots with respect to the health assessments are discussed in Chapter 9, they will be briefly described here with a focus on procedures and suggestions for other studies.

Since the first TILDA pilot was only conducted in Dublin, the option of a centre-based health assessment seemed sufficient. The expansion of the fieldwork to the whole country for the second pilot was addressed by the opening of a second dedicated centre in Cork city. In addition, early analysis of the first pilot results indicated that there was a systematic bias in the type of people participating in centre-based assessments in the opposite direction: the older and frailer part and the younger and working part of the population were under-represented in the centre-based assessments. As a result, it was decided to increase the available hours of the centre appointments to include evenings and Saturdays. Also, TILDA decided to introduce a subset of measures that could be conducted in a respondent's home. This would allow TILDA to have a core dataset of health data on a truly nationally representative sample of residents in Ireland. 
Health assessment data collection in dedicated centres and respondents' homes posed different challenges. In the case of the centres, the tests are located in fixed stations and the technology solutions are held on desktops, thereby improving physical security. Several of the novel tests are run using specific software that generates their own specific data output. In respondents' homes, only a subset of measures is captured. Among these, though, are two cognitive tests run on laptops that have to be uploaded for inclusion in the TILDA dataset. Furthermore, and intuitively, the fact that nurses were travelling to respondent's homes meant that lightweight, portable, and robust systems had to be implemented for that component of the study. As a result, different technology solutions were used in each instance.

The nurses also collect bloods from consenting respondents in both the centres and in respondent's homes. Since information relating to the blood samples had to go to the bloods processing lab in both instances, the bloods data transfer was handled via another technology solution.

The specifics in each case are described below.

\subsubsection{Health Assessments in TILDA Centres}

In the case of the centres, bespoke software was built by the Trinity Centre for High Performance Computing (TCHPC), based on a specification that incorporated the nurses' SOPs and the desired data output for each measure. In some cases, nurses input data directly into the TCHPC software interface while in other cases, the nurses conduct a measure on a bespoke software package, i.e. for the GAITRite or cardiovascular systems, and upload the appropriate files for inclusion in the TILDA dataset. For each respondent, the nurse initialises an encrypted USB key, collects information for that respondent at each station of measures, uploads the information for each station to the key and then transfers the complete encrypted data record to a Trinity server housed by TCHPC via a secure method. By the time the main wave of the study went live, the Cork centre had been fully set up to transfer data in this secure manner to TCHPC in Dublin, but it should be noted that assuring appropriate security measures required some time, planning, and an understanding of the requirements to uphold respondent confidentiality in all data transfers.

\subsubsection{Home-based Health Assessments}

In the case of home-based assessments, TILDA contracted with Crimson Tide to develop Personal Digital Assistant (PDA)-based software which the nurses could use both to track new assignments and set up appointments as well as to collect the results from the measures conducted on site. This software program was also designed to allow for the upload of files from the two cognitive tasks conducted in the home on laptops, namely the SART and CRT. 


\subsubsection{Bloods data}

Prior to taking a blood sample, the nurses capture information about the consent given by the respondent, the time since the respondent's last meal and whether the respondent was able to give sufficient blood for each of the three collection vials. This bloods module was also built by Crimson Tide into PDA's which were used at points of blood collection both in respondent homes and the dedicated TILDA centres. The information collected in this way is transmitted, in encrypted format, to a server hosted by Crimson Tide for TILDA, where data is re-formatted into an e-mail report for use by the processing lab and the bloods storage facility, ADP Pathology Storage Ltd. 


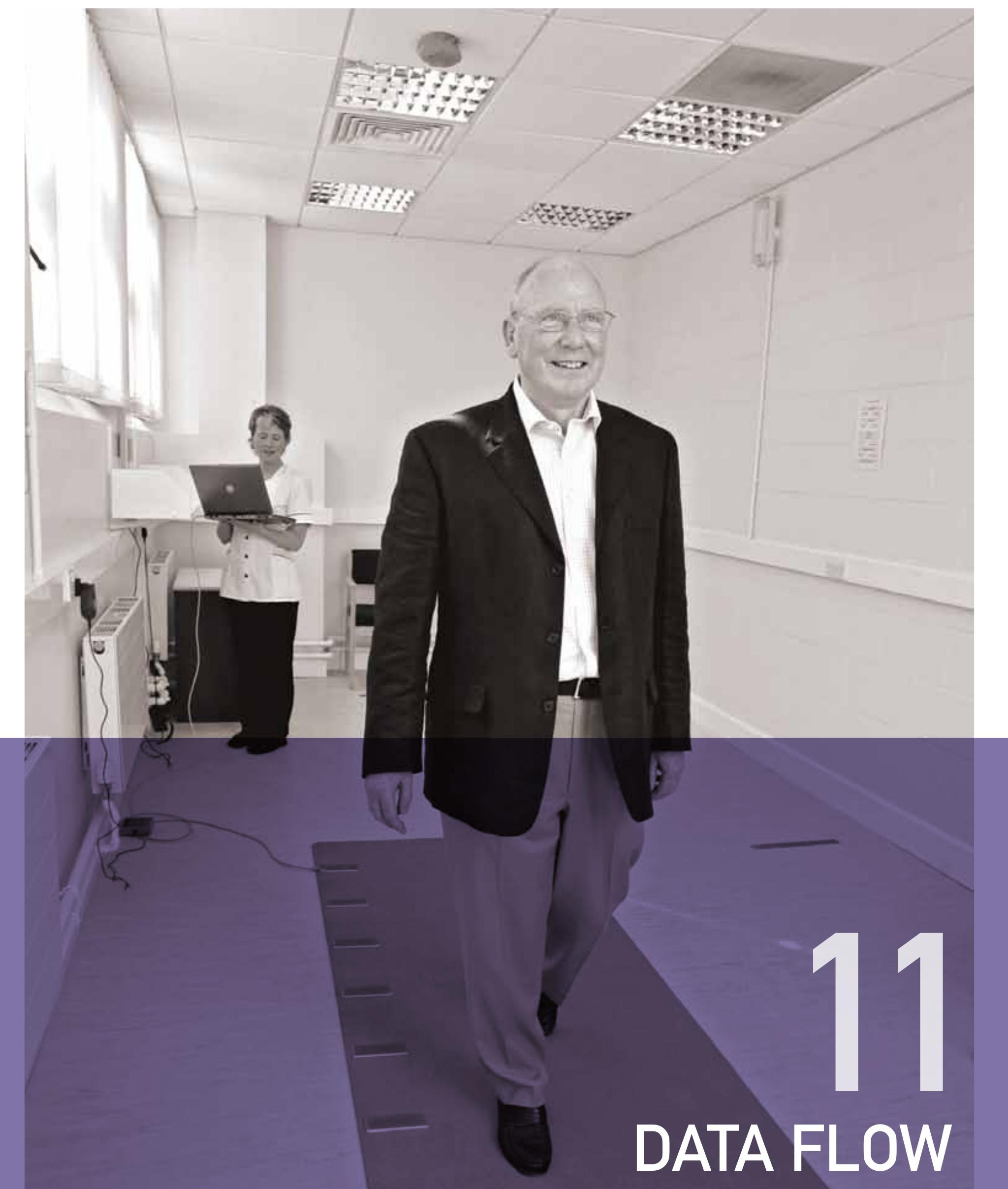




\section{data flow}

\subsection{Data Sources}

The TILDA data is generated using the tools and methods described in the preceding chapters. It is expected that data collection will continue throughout most of 2010 with the health assessment possibly continuing through early 2011. All data is transmitted to the TILDA Central Data Repository, as indicated in Fig. 11.1 below. There, it will be combined and documented for ease of use by the research community.

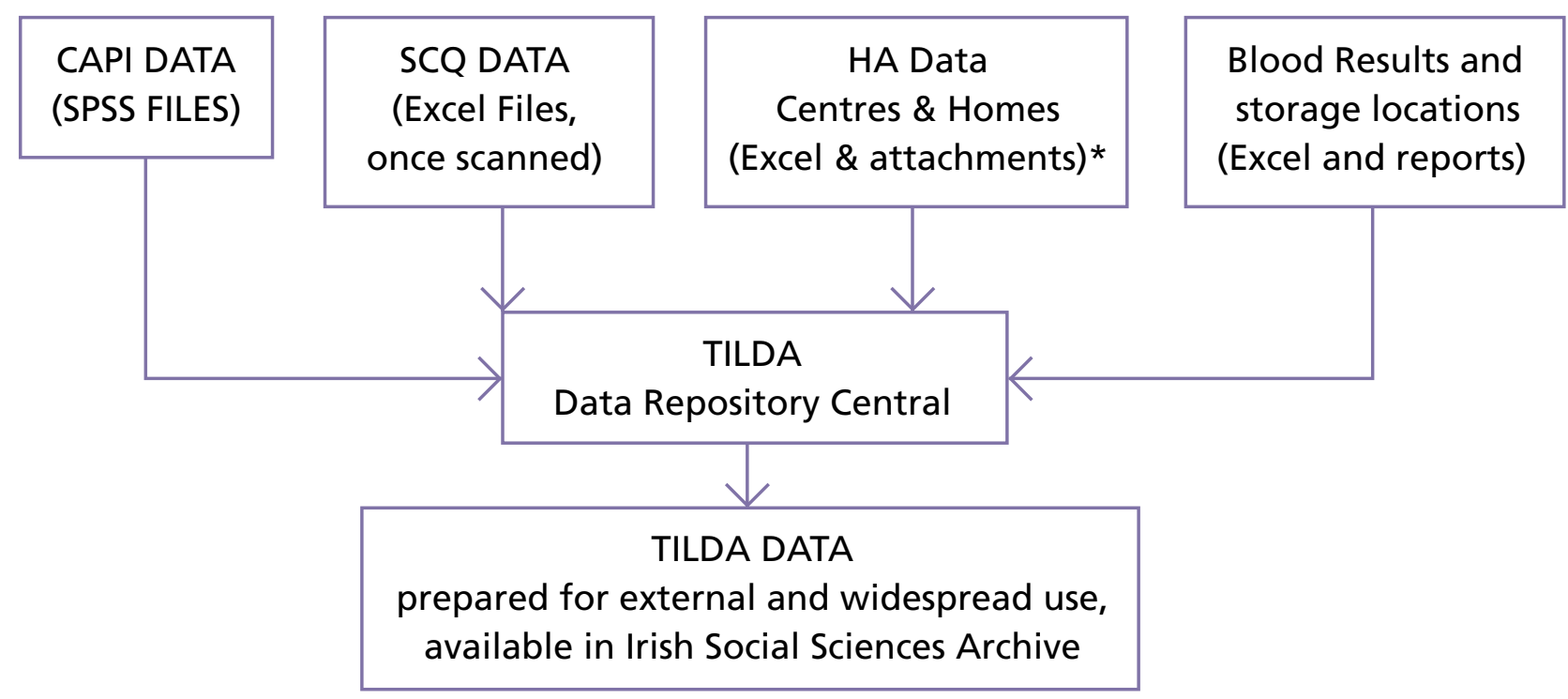

*Potentially identifiable data such as retinal photos and genetic results are kept separately from other data to preserve respondent anonymity and confidentiality, in accordance with guidelines and principles set by the Data Protection Commissioner.

\section{Figure 11.1 TILDA Data Flow}

After the data cleaning period, which is anticipated to take approximately one-year from the end of data collection, a TILDA dataset incorporating all core results will be made available via the Irish Social Sciences Data Archive held at University College Dublin.

However, certain results that have been deemed potentially identifiable will not appear in the archived dataset. In general, data which are potentially identifiable because of their nature, such as the retinal images and genetic codes, or because the level of aggregation is insufficient to guarantee respondent confidentiality, will not appear in the archived dataset. Request for 
datasets with a greater level of detail, a different level of aggregation, or inclusive of the potentially identifiable data, can be submitted to the TILDA Data Sharing subcommittee of the Steering Committee for review and consideration.

\subsection{Comments on Limitations of this Report}

The potential power of the TILDA dataset is immeasurable and this report has not done justice to the type or level of research inquiries that can be pursued with it. This report attempts to describe the TILDA study by its domains, (economic, health, and social, or health, wealth, and happiness, as they are sometimes called) in logical sequence as they were developed and in a way that may help individual researchers quickly find the information about the study that they need.

However, this report has two main limitations. First, it is focused on explaining the data collection choices implemented for the main wave, or baseline, of the study. As a result, the true power and novelty of a longitudinal Irish data set is not described. For example, TILDA will be able to track people's expectations and planning about retirement prior to making those decisions and see what occurs once people have in fact retired. Also, as TILDA tracks people over time, TILDA will provide insights into the risk factors and precursors of different types of disease and disability.

Secondly, what this report does not convey for the most part is the type of multi-disciplinary analysis that TILDA will facilitate and that can really provide learning for the international research community. For example, there are obvious effects of health on employment as well as community engagement on health. TILDA will provide the kind of comprehensive data that will allow such interdisciplinary research to take place. 

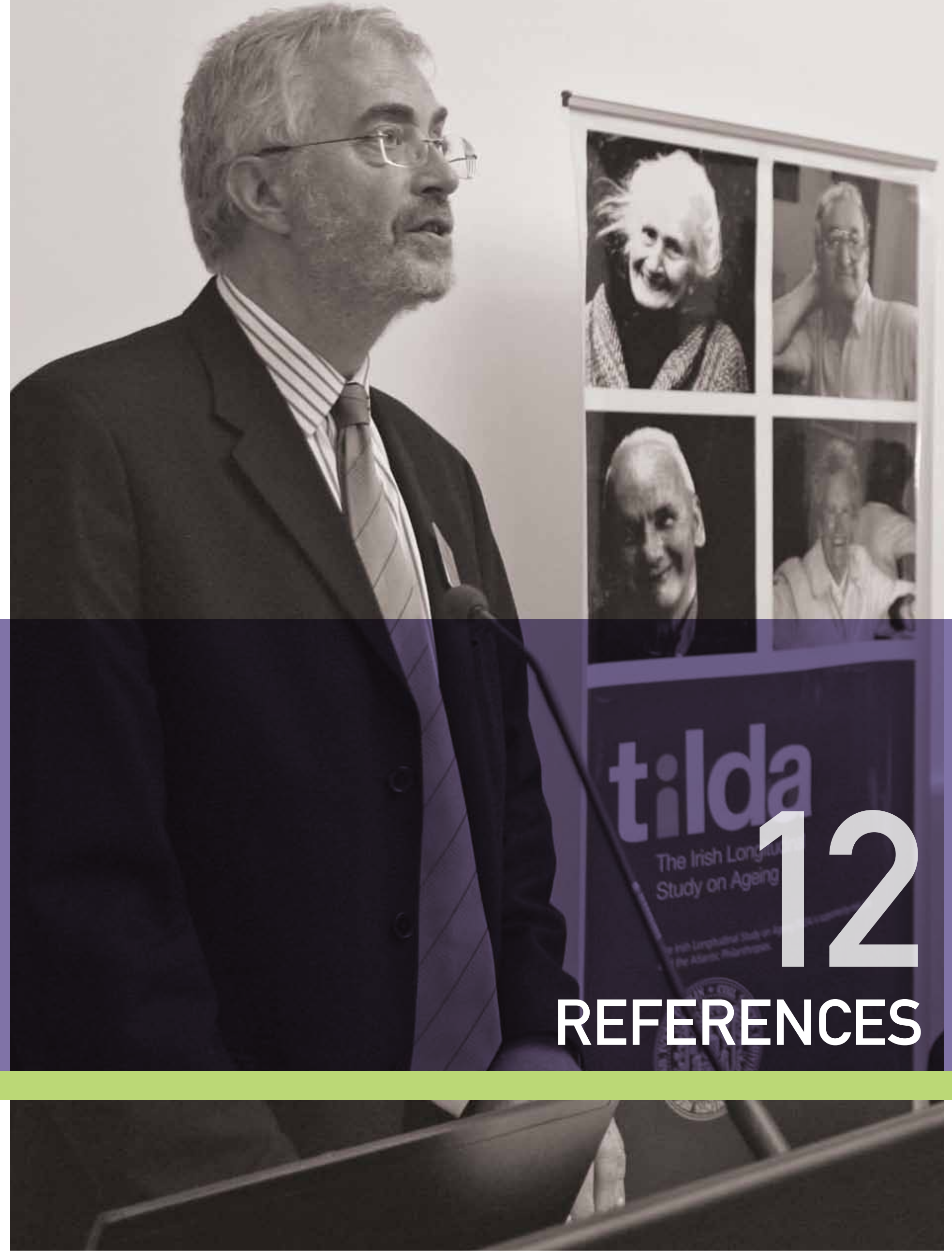


\section{references}

Alarcón, T., Bárcena, A., González-Montalvo, J.I., Penãlosa, C., \& Salgado, A. (1999). Factors predictive of outcome on admission to an acute geriatric ward.

Age Ageing 28, 429-432.

Almond, D., Edlund, L., Li, H., \& Zhang, J. (2007). Long-Term Effects Of The 1959-1961 China Famine: Mainland China and Hong Kong. NBER.

Ammirati, F., Colivicchi, F., Velardi, A., \& Santini, M. (2001). Prevalence and correlates of syncope-related traumatic injuries in tilt-induced vasovagal syncope. Ital Heart J., 2(1), 38-41.

Arber, S. \& Ginn, J. (1993). Gender and inequalities in health in later life. Social Science and Medicine 36(1), pp. 33-46.

Austin, B. A. (1983). Factorial structure of the UCLA Loneliness Scale. Psychol Rep, 53(3 Pt 1), 883-889.

Banks, J., Blundell, R., \& Smith, J. P. (2003). Financial Wealth Inequality in the United States and Great Britain. Journal of Human Resources, 38(2).

Barker, M., O’Hanlon, A., McGee, H. M., Hickey, A., \& Conroy, R. M. (2007). Cross-sectional validation of the Aging Perceptions Questionnaire: a multidimensional instrument for assessing self-perceptions of aging. BMC Geriatr, 7, 9.

Barlow, D. H. (1988). Anxiety and Its Disorders. New York: Guilford Press.

Bath, P. A., \& Deeg, D. (2005). Social engagement and health outcomes among older people: introduction to a special section European Journal of Ageing, 2 (1).

Beekman, A.T., Deeg, D.J., Van Limbeek, J., Braam, A.W., De Vries, M.Z., \& Van Tilburg, W. (1997). Criterion validity of the Center for Epidemiologic Studies Depression Scale (CES-D): results from a community-based sample of older subjects in the Netherlands. Psychol Med, 27, 231-5.

Bell, R. (1984). Item Nonresponse in Telephone Surveys: An Analysis of Who Fails to Report Income. Social Science Quarterly, 65, 207-215. 
Berkman, L. F., \& Syme, S. (1979). Social networks, host resistance, and mortality: a nine-year follow-up study of Alameda County residents. American Journal of Epidemiology, 109(2), 186-204.

Bernadt, M. W., Mumford, J., Taylor, C., Smith, B., \& Murray, R. M. (1982). Comparison of questionnaire and laboratory tests in the detection of excessive drinking and alcoholism. Lancet, 1(8267), 325-328.

Blake, A.J., Morgan, K., Bendall, M.J., Dallosso, H., Ebrahim, S.B.J., Arie, T.H.D., Fentem, P.H., \& Bassey, E.J. (1988). Falls by elderly people at home: prevalence and associated factors. Age Ageing 17, 365-372.

British Heart Foundation (2002), Database: Coronary Heart Disease Statistics, www.heartstats. org/datapage.asp?id=722, date accessed 07/10/2009.

British Medical Association and Royal Pharmaceutical Society of Great Britain. (2009) British National Formulary, 58 ed.

Brosschot, J. F., Van Dijk, E., \& Thayer, J. F. (2007). Daily worry is related to low heart rate variability during waking and the subsequent nocturnal sleep period. Int $J$ Psychophysiol, 63(1), 39-47.

Case, A., Fertig, A., \& Paxson, C. (2005). The lasting impact of childhood health and circumstance Journal of Health Economics, 24(2), 365-389.

Central Statistics Office. (2006). Quarterly National Household Survey Pensions update Quarter 1 2005. Dublin: Stationary Office.

Central Statistics Office. (2004). Population and Labour Force Projections 2006-2036. Dublin: Stationary Office.

Charney, D.S., Reynolds, C.F., Lewis, L., Lebowitz, B.D., Sunderland, T., Alexopoulos, G.S., et al. (2003). Depression and Bipolar Support Alliance consensus statement on the unmet needs in diagnosis and treatment of mood disorders in late life. Arch Gen Psychiatry, 60, 664-672.

Chia, E.M., Mitchell, P., Rochtchina, E., Foran, S., Golding, M., \& Wang, J.J.(2006) Association Between Vision and Hearing Impairments and Their Combined Effects on Quality of Life. Arch Ophthalmol, 124, 1465-1470.

Chiarelli, P.E., Mackenzie, L.A., \& Osmotherly, P.G.(2009).Urinary incontinence is associated with an increase in falls: a systematic review. Aust J Physiother, 55(2), 89-95.

Christelis, D., Jappelli, T., Paccagnella, O., \& Weber, G. (2006). Socio-Economic Status, Income, \& Wealth (from http://www.share-project.org/t3/share/fileadmin/AMANDA Praesentationen/Socio-Economics_Status_Income_Wealth.pdf, date accessed 20/11/2009. 
Clark, D. O., Callahan, C.M., \& Counsell, S.R. (2005). Reliability and validity of a steadiness score. Journal of the American Geriatrics Society, 53(9), 1582-6.

Clark, S., Youngman, L.D., Chukwurah, B., Palmer, A., Parish, S., Peto, R., \& Collins, R. (2004). Effect of temperature and light on the stability of fat-soluble vitamins in whole blood over several days: implications for epidemiological studies. Int J Epidemiol 33, 518-525.

Clark, S., Youngman, L.D., Palmer, A., Parish, S., Peto, R., \& Collins, R. (2003). Stability of plasma analytes after delayed separation of whole blood: implications for epidemiological studies. Int J Epidemiol 32, 125-130.

Cohen, S. (2004). Social relationships and health. American Psychology, 59(8), 676-684.

Cohen, S. (1988). Psychosocial models of the role of social support in the etiology of physical disease. Health Psychology, 7(3), 269-297.

Cohen, S., Kamarck, T., \& Mermelstein, R. (1983). A global measure of perceived stress. J Health Soc Behav, 24(4), 385-396.

Commission of the European Communities (2005). Council Decision 2005/600/EC of 12 July 2005 on guidelines for the employment policies of the Member States.

Craig, C.L., Marshall, A.L., Sjostrom, M., Bauman, A.E., Booth, M.L., Ainsworth, B.E., Pratt, M., Ekelund, U., Yngve, A., Sallis, J.F., \& Oja, P. (2003). International physical activity questionnaire: 12-country reliability and validity. Med Sci Sports Exerc, 35, 1381-95. 17.

Craske, M. G., Rapee, R. M., Jackel, L., \& Barlow, D. H. (1989). Qualitative dimensions of worry in DSM-III-R generalized anxiety disorder subjects and nonanxious controls. Behav Res Ther, 27(4), 397-402.

de Jong Gierveld, J., \& Havens, B. (2004). Cross-national comparisons of social isolation and loneliness: introduction and overview. Can J Aging, 23(2), 109-113.

Department of Social and Family Affairs. (2007). Green Paper on Pension. Dublin: Government Stationery Office.

Department of the Taoiseach. (2006) Towards 2016. Ten-Year Framework Social Partnership Agreement 2006-2015. Dublin: Government Stationery Office.

Drammeh, B.S., Schleicher, R.L., Pfeiffer, C.M., Jain, R.B., Zhang, M., \& Nguyen, P.H. (2008). Effects of Delayed Sample Processing and Freezing on Serum Concentrations of Selected Nutritional Indicators. Clin Chem 54, 1883-1891.

Elo, I., \& Preston, S. (1996). Educational differentials in mortality: United States, 1979-85. Social Science and Medicine, 42(1), 47-57. 
Ettner, S.L., Frank, R.G., \& Kessler, R.C. (1997). The impact of psychiatric disorders on labor market outcomes. Industrial and Labor Relations Review, 51(1), 64-81.

European Union Survey of Income and Living Conditions. (2001). Community Statistics on Income and Living Conditions.

Evandrou, M., \& Victor, C. R. (1989). Inequalities in Health in Later Life. Age and Ageing, 18(6), 387-391.

Fisher, G.G., Faul, J.D., Weir, D.R., \& Wallace, R.B. (2005). Documentation of Chronic Disease Measures in the Health and Retirement Study (HRSIAHEAD). HRS Documentation Report DR-009.

Folstein, M.F., Folstein, S.E., \& McHugh, P.R. (1975). „Mini-mental state“: a practical method for grading the cognitive state of patients for the clinician. J Psychiatr Res, 12, 189-198.

Fox, A. J., \& Goldblatt, P. O. (1982). Selection of Mortality Differentials. Journal of Epidemiology and Community, 36, 69-79.

Fulton, M.M., \& Allen, E.R.(2005). Polypharmacy in the elderly: a literature review. J Am Acad Nurse Pract 17:123-32. PMID 15819637.

Gannon, B., O'Shea, E., \& Hudson, E. (2007). The Economic Cost of Falls and Fractures in People aged 65 and over in Ireland. Technical Report to NCAOP/HSE/DOHC. Irish Centre for Social Gerontology National University of Ireland, Galway, Ireland.

George, L. K., D.B.Larson, Koenig, H. G., \& McCullough, M. E. (2000). Spirituality and health: what we know, what we need to know. Journal of Social and Clinical Psychology, 19(102-116).

Glass, T. A., de Leon, C. M., Marottoli, R. A., \& Berkman, L. F. (1999). Population based study of social and productive activities as predictors of survival among elderly Americans. BMJ, 319(7208), 478-483.

Goldman, N., Korenman, S., \& Weinstein, R. (1995). Marital status and health among theelderly. Social Science and Medicine, 40, 1717-1730.

Grundy, E., \& Henretta, J. (2006). Between elderly parents and adult children: a new look at the intergenerational care provided by the 'sandwich generation'. Ageing and Society 26(5), 707-722.

Haider, S.I., Johnell, K., Thorslund, M., \& Fastbom, J. (2007). Trends in polypharmacy and potential drug-drug interactions across educational groups in elderly patients in Sweden for the period 1992 - 2002. International Journal of Clinical Pharmacology and Therapeutics 45 (12): 643-653. PMID 18184532. 
Hajjar, R.R. (2004). Psychosocial impact of urinary incontinence in the elderly population. Clinics in Geriatric Medicine, Volume 20, Issue 3, Pages 553-564.

Hamilton, M. (1959). The assessment of anxiety states by rating. Br J Med Psychol, 32(1), 50-55.

Hermalin, A. (2000). Challenges to Comparative Research on Intergenerational Transfers. Ann Arbor: University of Michigan.

Health Service Executive.(2009). Primary Care Reimbursement Service. http://www.hse.ie/eng/ staff/PCRS/About_PCRS/, Date accessed 13/11/09.

Health Service Executive, (2008). Strategy to Prevent Falls and Fractures in Ireland's Ageing Population. http://www.hse.ie/eng/services/Publications/services/Older/Strategy_to_ Prevent_Falls_and_Fractures_in_Ireland\%E2\%80\%99s_Ageing_Population_-_Full_report. pdf, date accessed 02/12/2009.

Hill, K., Schwarz, J., Kalogeropoulos, A., \& Gibson, S.(1996). Fear of falling revisited. Archives of Physical Medicine and Rehabilitation 77, 1025-1029.

Hippler, H.J., \& Hippler, G. (1986). Reducing Refusal Rates in the Case of Threatening Questions: The 'Door-in-the-Face' Technique. Journal of Official Statistics, 2, 25-33.

Hodges, W. F., \& Spielberger, C. D. (1969). An indicant of trait or state anxiety? J Consult Clin Psychol, 33(4), 430-434.

House, J., Robbin, C., \& Metzner, H. (1982). The association of social relationships and activities with mortality: prospective evidence from the Tecumseh community health study American Journal of Epidemiology 116(1), 123-140.

Hu, Y., \& Goldman, N. (1990). Mortality Differentials by Marital Status: An International Comparison. Demography, 27(2), 233-250.

Hyde, M., Wiggins, R. D., Higgs, P., \& Blane, D. B. (2003). A measure of quality of life in early old age: the theory, development and properties of a needs satisfaction model (CASP-19). Aging Ment Health, 7(3), 186-194.

Idler, E.L., \& Angel, R.J. (1990). Self-rated health and mortality in the NHANES-I epidemiologic follow-up study. Am J Public Health, 80, 446-52.

Inter-Departmental Committee on the Care of the Aged. (1968). The Care of the Aged. Dublin: Stationery Office.

International Physical Activity Questionnaires. (2005). Guidelines for data processing and analysis of the International Physical Activity Questionnaire (IPAQ) - Short form: scoring protocol. http://www.ipaq.ki.se./ipaq.htm, date accessed 12/10/2009. 
Jacqmin-Gadda, H., Fourrier, A., Commenges, D., \& Dartigues, J.F. (1998). Risk factors for fractures in the elderly. Epidemiology 9, 417-423.

Jenkins, K.R., Ofstedal, M.B., \& Weir, D. (2008). Documentation of Health Behaviours and Risk Factors Measured in the Health and Retirement Study (HRSIAHEAD). HRS Documentation Report DR-010.

Juster, T., \& Smith, J. P. (1997). Improving the Quality of Economic Data: Lessons from the HRS and AHEAD. Journal of the American Statistical Association, 92.

Kaplan, G.A., \& Camacho, T. (1983). Perceived health and mortality: a nine-year follow-up of the human population laboratory cohort. Am J Epidemiol ,117, 292-304.

Kempen, G.I.J.M., Todd, C.J., Van Haastregt, J.C.M., et al. (2006). Cross-cultural validation of the Falls Efficacy Scale International (FES-I) in older people: results from Germany, the Netherlands and the UK were satisfactory. Disabil Rehabil in press.

Kennickell, A. B. (1997). Multiple Imputation and Disclosure Protection: The Case of the 1995 Survey of Consumer Finances. The Federal Reserve Board.

Kish, L. (1965). Survey Sampling. New York: John Wiley and Sons.

Kitchens, J. M. (1994). Does this patient have an alcohol problem? JAMA, 272(22), 1782-1787.

Knight, R. G., Chisholm, B. J., Marsh, N. V., \& Godfrey, H. P. (1988). Some normative, reliability, and factor analytic data for the revised UCLA Loneliness Scale. J Clin Psychol, 44(2), 203-206.

Koenig, H. G. (2000). Handbook of Religion and Health. Oxford: Oxford University Press.

Korenman, S., Goldman, N. (1993) Health and mortality differentials by marital status at older ages: economics and gender. Working paper No. 93-8. Office of Population Research, Princeton, NJ.

Kvaal, K., Ulstein, I., Nordhus, I. H., \& Engedal, K. (2005). The Spielberger State-Trait Anxiety Inventory (STAI): the state scale in detecting mental disorders in geriatric patients. Int J Geriatr Psychiatry, 20(7), 629-634.

Lebowitz, B.D., Pearson, J.L., Schneider, L.S., Reynolds, C.F., Alexopoulos, G.S., Bruce, M.L., et al. (1997). Diagnosis and treatment of depression in late life. Consensus statement update. JAMA 278, 1186-1190.

Lennartsson, C., \& Silverstein, M. (2001). Does engagement with life enhance survival of elderly people in Sweden? The role of social and leisure activities. J Gerontol B Psychol Sci Soc Sci, 56(6), S335-342. 
Leserman, J. (2000). The effects of depression, stressful life events, social support, and coping on the progression of HIV infection. Curr Psychiatry Rep, 2(6), 495-502.

Lillard, L. A., \& Panis, C. (1996). Marital status and mortality: the role of health. Demography, 33, 313-327.

Lindenberger, E.C., Landefeld, C.S., Sands, L.P., et al. (2003). Unsteadiness reported by older hospitalized patients predicts functional decline. J Am Geriatr Soc, 51, 621-626.

Linton, A., Garber, M., Fagan, N.K., Peterson, M.R. ( 2007). Examination of multiple medication use among TRICARE beneficiaries aged 65 years and older. J. Manag. Care Pharm. 13 (2): 155-162.

Magaziner, J. Cadigan, D.A., Fedder, D.O. \& Hebel, J.R. (1989). Medication use and functional decline among community dwelling older women. J. Aging Health $1,470-484$.

Maier, H., \& Klumb, P. L. (2005). Social participation and survival at older ages: is the effect driven by activity content or context? European Journal of Ageing, 2(1).

Manton, K. G. (1998). Current trends in healthy life expectancy and declining disability rates in the USA. Paper presented at the World Health Report 1998: Life in the 21st Century? Vision for All, Geneva, Switzerland.

Marmot, M., Banks, J., Blundell, R., Lessof, C., Nazroo, J. (2002). Health, wealth and lifestyles of the older population in England. The 2002 English Longitudinal Study of Ageing.

Mayfield, D., McLeod, G., \& Hall, P. (1974). The CAGE questionnaire: validation of a new alcoholism screening instrument. Am J Psychiatry, 131(10), 1121-1123.

Mendes de Leon, C. F. (2005). Social engagement and successful aging. European Journal of Ageing, 2(1), 64.

Meyer, T. J., Miller, M. L., Metzger, R. L., \& Borkovec, T. D. (1990). Development and validation of the Penn State Worry Questionnaire. Behav Res Ther, 28(6), 487-495.

Miller, W. R. (1998). Researching the spiritual dimensions of alcohol and other drug problems (Vol. 93): Taylor\&Francis Group.

Mineau, G., Smith, K., \& L. (2002). Historical Trends of Survival among Widows and Widowers. Social Science and Medicine., 54, 245-254.

Medical Research Council Cognitive Function and Ageing (MRC CFA) Study (1998). 'Cognitive function and dementia in six areas of England and Wales: the distribution of MMSE and prevalence of GMS organicity level in the MRC CFA Study', Psychological Medicine, 28, 319-335. 
Murphy, S.L., Dubin, J.A., Gill, T.M. (2003). The development of fear of falling among community-living older women: predisposing factors and subsequent fall events. J Gerontol A Biol Sci Med Sci 58, M943-7.

National Council on Ageing and Older People. (2003) NCAOP Report 77: Healthy Ageing in Ireland: Policy, Practice and Evaluation.

Netuveli, G., Wiggins, R. D., Hildon, Z., Montgomery, S. M., \& Blane, D. (2006). Quality of life at older ages: evidence from the English longitudinal study of aging (wave 1). J Epidemiol Community Health, 60(4), 357-363.

Netuveli, G., Wiggins, R.D., Hildon, Z., Montgomery, S.M., Blane, D. (2005). Functional limitation in long standing illness and quality of life: evidence from a national survey. BRIT MED J. 331,1382-+.

Nolan, B., Whelan C.T., Maitre, B. (2005). Trends in Welfare for Vulnerable Groups, Ireland 1994-2001. Policy Research Series Paper No. 56, Dublin: The Economic and Social Research Institute.

Nusbaum, N.J. (1999). Aging and sensory senescence. South Med J., 92(3), 267-75.

O'Connell, H., Chin, A.V., Cunningham, C., Lawlor, B.A. (2004). Recent developments: Suicide in older people. British Medical Journal, 329, 895-899.

Organisation for Economic Co-Operation and Development. (2006). Live Longer, Work Longer: Ageing and Employment Policies.

Organisation for Economic Co-Operation and Development. (2001). Mobility Needs and Safety Problems of an Ageing Society. Paris: OECD.

Ofstedal, M.B., Fisher, G.G., Herzog, A.R. (2005). Documentation of Cognitive Functioning Measures in the Health and Retirement Study. HRS Documentation Report DR-006.

Ó’Muircheartaigh, C., Smith, S. (2007). NSHAP (National Social Life, Health, and Aging Project) Wave 1 methodology report. Chicago: National Opinion Research Center (NORC).

Pachana, N. A., Byrne, G. J., Siddle, H., Koloski, N., Harley, E., \& Arnold, E. (2007). Development and validation of the Geriatric Anxiety Inventory. Int Psychogeriatr, 19(1), 103-114.

Pappas, G., Queen, S., Hadden, W., \& Fisher, G. (1993). The Increasing Disparity in Mortality between Socioeconomic Groups in the United States, 1960 and 1986. The New England Journal of Medicine, 329 103-109.

Preston, S. H., Hill, M. E., \& Drevenstedt, G. L. (1998). Childhood conditions that predict survival to advanced ages among African-Americans Social Science and Medicine, 47(9), 1231-1246. 
Prince, M.J., Reischies, F., Beekman, A.T.F, et al. (1999). Development of the EURO-D scale-a European Union initiative to compare symptoms of depression in 14 European centres. Br J Psychiatr 174, 330-338.

Qato, D.M., Alexander, G.C., Conti, R.M., Johnson, M., Schumm, P., \& Lindau, S.T.(2008). Use of Prescription and Over-the-counter Medications and Dietary Supplements among Older Adults in the United States. JAMA 300, 2867-2878.

Radloff, L.S. (1977). The CES-D scale: a self-report depression scale for research in the general population. Applied Psychological Measurement 1, 385-401.

Reynolds, J., \& Ross, C. (1998). Social Stratification and Health. Social Problems, 45, 221-247.

Rose, M.S., Koshman, M.L., Spreng, S., \& Sheldon, R. (2000). The relationship between healthrelated quality of life and frequency of spells in patients with syncope. $J$ Clin Epidemiol 53, 1209-16.

Rosengren, A., Orth-Gomer, K., Wedel, H., \& Wilhelmsen, L. (1993). Stressful life events, social support, and mortality in men born in 1933. BMJ, 307(6912), 1102-1105.

Russell, D., Peplau, L. A., \& Cutrona, C. E. (1980). The revised UCLA Loneliness Scale: concurrent and discriminant validity evidence. J Pers Soc Psychol, 39(3), 472-480.

Russell, D. W. (1996). UCLA Loneliness Scale (Version 3): reliability, validity, and factor structure. J Pers Assess, 66(1), 20-40.

Ruhs, M. (2004). Managing the Immigration and Employment of Non-EU nationals in Ireland. : The Policy Institute. Trinity College Dublin. Dublin.

Smith, J. (1999). Healthy Bodies and Thick Wallets: The Dual Relation between Health and Economic Status The Journal of Economic Perspectives, 13(2), 145-166.

Smith, J. P., \& Kington, R. (1997). Demographic and Economic Correlates of Health in Old Age Demography, 34(1), 159-170.

Smith, K., \& Zick, C. D. (1994). Risk of mortality following widowhood: age and sex differences by mode of Death. Social Biology, 43(1-2).

Soldo, B., \& Hill, M. S. (1993). Intergenerational transfers: Economic, demographic, and social perspectives. In G. L. M. M. P. Lawton (Ed.), Annual review of gerontology and geriatrics: Focus on kinship, aging, and social change. New York: Springer.

Steen, G., Sonn, U., Hanson, A.B., \& Steen, B. (2001). Cognitive function and functional ability: A cross-sectional and longitudinal study at ages 85 and 95 in a nondemented population. Aging (Milano) 13(2), 68-77. 
The Working Party on Services for the Elderly. (1988). The Years Ahead-A Policy for the Elderly. Dublin: Government Publications.

Timonen, V., Doyle, M., \& Prendergast, D. (2006). No Place Like Home: Domiciliary Care Services For Older People In Ireland: Liffey Press.

Tinetti, M.E., Richman, D., \& Powell, L. (1990). Falls efficacy as a measure of fear of falling. J Gerontol Psy Sci.,45, P239-P243.

United Nations Programme on Ageing. (2007) Research Agenda on Ageing for the 21st Century 2007 Update. A joint project of the United Nations Programme on Ageing and the International Association of Gerontology and Geriatrics. Retrieved online February 16, 2010 from http://www.un.org/esa/socdev/ageing/documents/AgeingResearchAgenda6.pdf, date accessed 16/02/10.

United Nations. (2002). Report of the Second World Assembly on Ageing: Madrid International Plan of Action on Ageing, 2002. http://www.un.org/esa/socdev/ageing/ madrid_intlplanaction.html, date accessed 16/02/10.

Valete-Rosalino, C.M., Rozenfeld, S. (2005). Auditory screening in the elderly: comparison between self-report and audiometry. Braz J Otorhinolaryngol, 71(2), 193-200.

Vision Rehabilitation Laboratory, Schepens Eye Research Institute, Boston. (2009). Driving Habits Questionnaire. www.eri.harvard.edu/faculty/peli/shared/index_page.htm, date assessed 10/12/2009.

Whelan, B.J. (1979). RANSAM: A National Random Sampling Design for Ireland. The Economic and Social Review 10(2).

World Health Organization. (1998). Development of the World Health Organization WHOQOLBREF quality of life assessment. The WHOQOL Group. Psychol Med, 28(3), 551-558.

Wilson, B., Cockburn, J., \& Baddeley, A.D. (1985). The Rivermead Behavioural Memory Test, Reading. Thames Valley Test Company.

Wolf, D. A., Freedman, V., \& Soldo, B. J. (1997). The Division of Family Labor: Care for Elderly Parents. The Journal of Gerontology Series B, 102-109.

Yardley, L., Todd, C., Beyer, N., et al. (2005). Development and initial validation of the Falls Efficacy Scale International (FES-I). Age Ageing 34, 614-9.

Young, D.S., \& Bermes, E.W. (1999). Specimen collection and processing: sources of biological variation. In: Burtis CA, Ashwood ER (eds.). Tietz Textbook of Clinical Chemistry. Philadelphia: WB Saunders Company, pp.42-72.

Zigmond, A. S., \& Snaith, R. P. (1983). The hospital anxiety and depression scale. Acta Psychiatr Scand, 67(6), 361-370. 


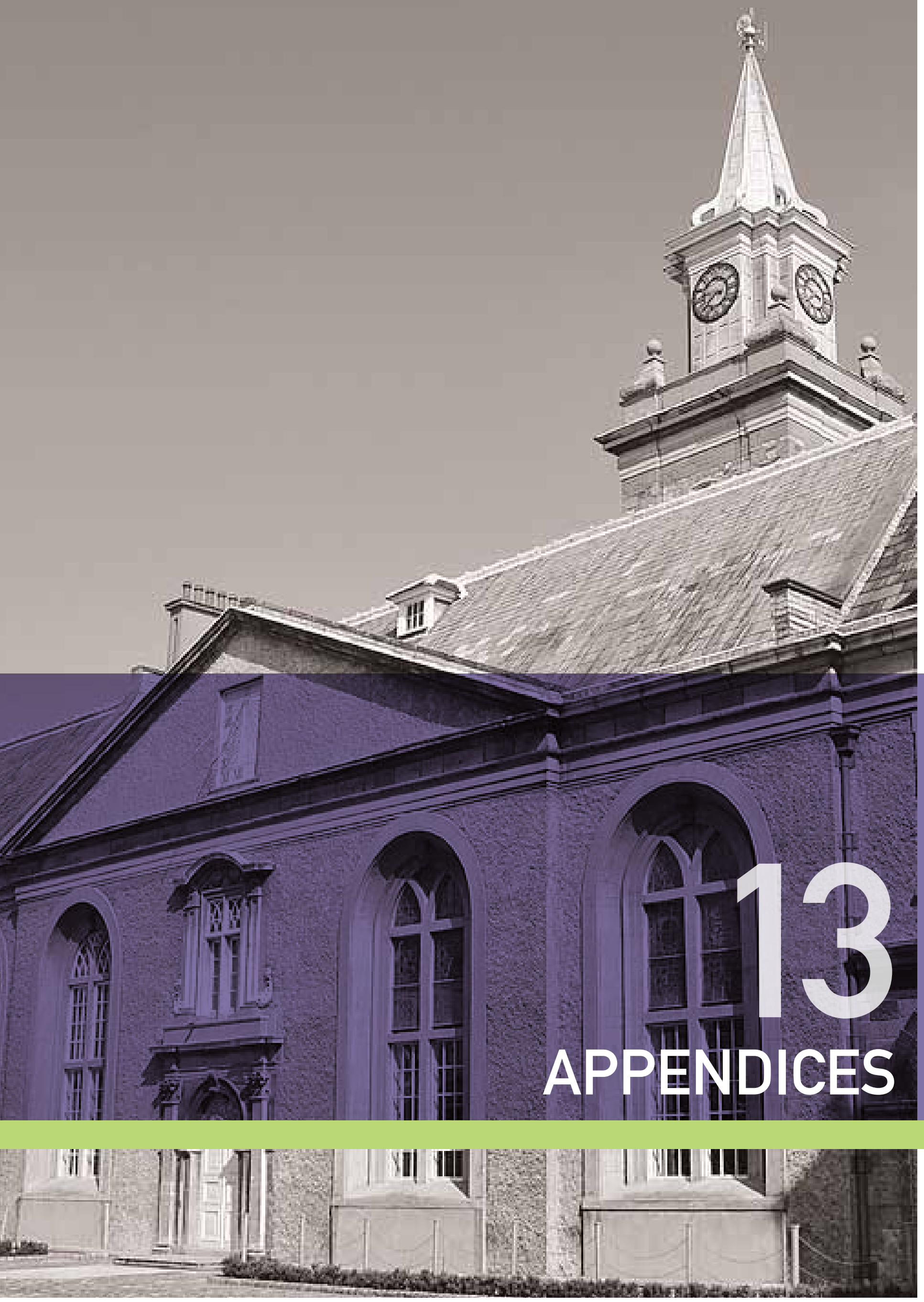




\section{appendices}

\section{Appendix 1: Comparison of Sampling Frames}

As described in Chapter one, TILDA had to choose between two sampling frames: the RANSAM system, based on the Geodirectory, and the Register of names and addresses of persons with Personal Public Service (PPS) Numbers. This appendix describes the main advantages and disadvantages of each possibility.

The RANSAM system is based on a listing of all residential addresses in Ireland. This list is kept under constant review and is regularly updated by its compilers, the national Postal Service (An Post) and the Ordnance Survey.

The main advantages of the RANSAM system are:

(a) It is a comprehensive listing of all addresses in Ireland that allows residential addresses to be distinguished, and is the only national listing available for this purpose. It is regularly updated.

(b) It has been used for numerous surveys and its properties are well known. The system is fully computerised and generates samples with minimum delay and a high degree of accuracy.

(c) It permits the selection of multi-stage samples of addresses with the possibility of stratification by various social and geographical variables at the first stage.

(d) It generates equal probability (self-weighting) samples of addresses at the first stage.

(e) It permits clustering of first stage sampling units.

(f) It incorporates an exact geocode for each address which facilitates interviewers in locating the selected address and also allows analysis of the sample by various levels of geographical location.

RANSAM does, however, have a number of defects in a study which aims to represent the TILDA target population i.e. "all adults aged 50 and over, and their spouses of any age, resident in private households in Ireland at the date of the survey".

\section{Its principal defects for the purposes of generating a sample for TILDA are:}

(a) About half of the addresses listed do not contain a person aged 50 or over. Since the ages of the residents are not known a priori, the interviewers must screen at least twice as many addresses as there are required interviews. This raises field costs 
appreciably. However, the addresses are usually adjacent to each other in clusters, which means that the cost of screening is much less than proportionate to that of achieved interviews.

(b) Since the respondent names are not known, preliminary letters must be addressed to "The Resident" or "The Occupant". This impersonal mode of address may reduce respondents' inclination to participate.

(c) The list contains some addresses which prove to be non-residential when visited. ESRI experience with the Geodirectory across a range of surveys indicates that this problem is not widespread, amounting to less than 5 per cent of addresses. It should also be noted that it does not give rise to sample bias, but only to slightly increased field costs due to fruitless calls by interviewers.

(d) In rural areas, up to 40 or 50 per cent of the addresses may be "non-unique" i.e. only distinguishable by reference to the resident's name (which is available to the postal authorities on most items of mail but is not included on the Geodirectory) or by reference to a geocode, combined with elaborate mapping and/or local information. However, the second pilot of TILDA pioneered the use of Satellite Navigation devices to lead interviewers directly to the dwelling in question. This procedure proved very satisfactory in the field.

The Personal Public Service (PPS) Number system has the major advantages that (a) it lists individuals by name and (b) their date of birth is known. This means that interviewers can go directly to the relevant respondent's dwelling and personalised letters can be sent by post to respondents before the interviewer calls. Thus, a sample where every member of the target age group has an equal probability of selection could be selected from the Register (technically known as an epsem sample).

\section{The main defects of the PPS Register for sampling purposes are:}

(a) it contains no geographical information beyond address (i.e. county sub-divisions such as District Electoral Divisions are not known for each address). This means that travelling time and costs are unknown and are likely to be high.

(b) The system has not been used before for sampling purposes so that its characteristics for this purpose are not known (e.g. extent of missing elements (if any), duplication, invalid elements, poor quality addresses and so on).

(c) It would include people resident in institutions and in private dwellings.

(d) The list is not publicly available and a range of steps must be taken to ensure complete compliance with data protection and confidentiality requirements. 


\section{Appendix 2: Measures of Control, Autonomy, Self-actualisation and Pleasure, the CASP-19}

CASP-19

\begin{tabular}{|c|c|}
\hline Control & $\begin{array}{l}\text { My age prevents me from doing the things I would like to } \\
\text { I feel that what happens to me is out of my control } \\
\text { I feel free to plan for the future } \\
\text { I feel left out of things }\end{array}$ \\
\hline Autonomy & $\begin{array}{l}\text { I can do the things that I want to do } \\
\text { Family responsibilities prevent me from doing what I want to do } \\
\text { I feel that I can please myself what I can do } \\
\text { My health stops me from doing the things I want to do } \\
\text { Shortage of money stops me from doing the things I want to do }\end{array}$ \\
\hline Pleasure & $\begin{array}{l}\text { I look forward to each day } \\
\text { I feel that my life has meaning } \\
\text { I enjoy the things that I do } \\
\text { I enjoy being in the company of others } \\
\text { On balance I look back on my life with a sense of happiness }\end{array}$ \\
\hline Self-Realisation & $\begin{array}{l}\text { I feel full of energy these days } \\
\text { I choose to do things I have never done before } \\
\text { I feel satisfied with the way my life has turned out } \\
\text { I feel that life is full of opportunities } \\
\text { I feel that the future looks good for me }\end{array}$ \\
\hline
\end{tabular}




\section{Appendix 3: Ageing Perceptions Questionnaire}

\section{Ageing Perceptions Questionnaire (APQ)}

We are interested in your own personal views and experiences about getting older. Please indicate your views on the following statements (strongly disagree, disagree, neither agree nor disagree, agree, or strongly agree). Mark the response that best describes your view for each statement.

\begin{tabular}{|c|c|c|c|c|c|}
\hline $\begin{array}{l}\text { PLEASE TICK ONE BOX PER LINE WHICH BEST } \\
\text { SHOWS HOW YOU FEEL ABOUT EACH STATEMENT }\end{array}$ & $\begin{array}{l}\text { Strongly } \\
\text { Disagree }\end{array}$ & Disagree & Neither & Agree & $\begin{array}{l}\text { Strongly } \\
\text { Agree }\end{array}$ \\
\hline 1. I am conscious of getting older all of the time & 1 & 2 & 3 & 4 & 5 \\
\hline 2. I am always aware of my age & 1 & 2 & 3 & 4 & 5 \\
\hline 3. I always classify myself as old & 1 & 2 & 3 & 4 & 5 \\
\hline 4. I am always aware of the fact that I am getting older & $1 \square$ & 2 & $3 \square$ & 4 & $5 \square$ \\
\hline 5. I feel my age in everything that I do & 1 & 2 & $3 \square$ & 4 & 5 \\
\hline 6. As I get older I get wiser & 1 & 2 & $3 \square$ & 4 & 5 \\
\hline 7. As I get older I continue to grow as a person & 1 & 2 & 3 & 4 & 5 \\
\hline 8. As I get older I appreciate things more & $1 \square$ & 2 & $3 \square$ & 4 & $5 \square$ \\
\hline $\begin{array}{l}\text { 9. I get depressed when I think about how } \\
\text { ageing might affect the things that I can do }\end{array}$ & 1 & 2 & $3 \square$ & 4 & $5 \square$ \\
\hline 10. The quality of my social life in later years depends on me & 1 & 2 & 3 & 4 & 5 \\
\hline 11. The quality of my relationships with others in later life depends on me & 1 & 2 & $3 \square$ & 4 & 5 \\
\hline 12. Whether I continue living life to the full depends on me & $1 \square$ & 2 & $3 \square$ & 4 & 5 \\
\hline $\begin{array}{l}\text { 13. I get depressed when I think about the effect } \\
\text { that getting older might have on my social life }\end{array}$ & 1 & 2 & 3 & 4 & 5 \\
\hline 14. As I get older there is much I can do to maintain my independence & $1 \square$ & 2 & 3 & 4 & 5 \\
\hline 15. Whether getting older has positive sides to it depends on me & $1 \square$ & 2 & $3 \square$ & 4 & 5 \\
\hline 16. Getting older restricts the things that I can do & 1 & 2 & $3 \square$ & 4 & 5 \\
\hline 17. Getting older makes me less independent & $1 \square$ & 2 & $3 \square$ & 4 & 5 \\
\hline 18. Getting older makes everything a lot harder for me & $1 \square$ & 2 & $3 \square$ & 4 & 5 \\
\hline 19. As I get older I can take part in fewer activities & $1 \square$ & 2 & 3 & 4 & 5 \\
\hline 20. As I get older I do not cope as well with problems that arise & $1 \square$ & 2 & $3 \square$ & 4 & 5 \\
\hline 21. Slowing down with age is not something I can control & $1 \square$ & 2 & 3 & 4 & 5 \\
\hline 22. How mobile I am in later life is not up to me & $1 \square$ & 2 & $3 \square$ & 4 & 5 \\
\hline 23. I have no control over whether I lose vitality or zest for life as I age & $1 \square$ & 2 & $3 \square$ & 4 & $5 \square$ \\
\hline $\begin{array}{l}\text { 24. I have no control over the effects which } \\
\text { getting older has on my social life }\end{array}$ & 1 & 2 & 3 & 4 & 5 \\
\hline 25. I get depressed when I think about getting older & $1 \square$ & $2 \square$ & $3 \square$ & 4 & 5 \\
\hline $\begin{array}{l}26 . \text { I worry about the effects that getting older } \\
\text { may have on my relationships with others }\end{array}$ & 1 & 2 & 3 & 4 & 5 \\
\hline $\begin{array}{l}\text { 27. I go through cycles in which my experience } \\
\text { of ageing gets better and worse }\end{array}$ & 1 & 2 & 3 & 4 & 5 \\
\hline 28. My awareness of getting older comes and goes in cycles & $1 \square$ & 2 & 3 & 4 & 5 \\
\hline 29. I feel angry when I think about getting older & $1 \square$ & 2 & $3 \square$ & 4 & 5 \\
\hline 30. I go through phases of feeling old & 1 & 2 & 3 & 4 & 5 \\
\hline 31. My awareness of getting older changes a great deal from day to day & $1 \square$ & 2 & $3 \square$ & 4 & $5 \square$ \\
\hline 32. I go through phases of viewing myself as being old & $1 \square$ & $2 \square$ & $3 \square$ & $4 \square$ & $5 \square$ \\
\hline
\end{tabular}




\section{The Ageing Perceptions Questionnaire (APQ)}

\section{Scoring Key}

\section{a) Views about getting older}

For these seven subscales, items are rated on a 5-point scale ranging from 'strongly disagree' (1), 'disagree' (2), 'neither agree nor disagree' (3) , 'agree' (4) and 'strongly agree' (5). With the exception of control negative, subscales items are scored from 1 to 5 . The mean score for each subscale is calculated. Higher scores are indicative of greater endorsement of a specific perception.

Timeline acute/chronic:

Timeline cyclical:

Emotional Representations:

Control positive:

Control negative:

Consequences positive:

Consequences negative:
Mean of items 1, 2, 3, 4, 5

Mean of items 27, 28, 30, 31, 32

Mean of items 9, 13, 25, 26, 29

Mean of items 10, 11, 12, 14, 15

Mean of items 21, 22, 23, 24 (items are reverse-scored)

Mean of items $6,7,8$

Mean of items 16, 17, 18, 19, 20 


\section{Appendix 4: TILDA Health Working Groups}

\begin{tabular}{|c|c|}
\hline Working Group & Participants \\
\hline Neuropsychological & $\begin{array}{l}\text { Professor RoseAnne Kenny* } \\
\text { Professor lan Robertson* } \\
\text { Professor Brian Lawlor* } \\
\text { Dr. Robert Coen* } \\
\text { Dr. Hilary Cronin* } \\
\text { Dr. Patricia Kearney* } \\
\text { Claire O'Regan* }\end{array}$ \\
\hline Cardiovascular & $\begin{array}{l}\text { Professor RoseAnne Kenny* } \\
\text { Professor Ian Graham* } \\
\text { Professor Ivan Perry }{ }^{*} \\
\text { Dr. Gabrielle McKee* } \\
\text { Dr. Hilary Cronin* } \\
\text { Dr. Patricia Kearney* } \\
\text { Claire O’Regan* }\end{array}$ \\
\hline Gait, Balance \& Sensory & $\begin{array}{l}\text { Professor RoseAnne Kenny* } \\
\text { Dr. Frances Horgan } \\
\text { Dr. Fiona Newell* } \\
\text { Dr. Hilary Cronin* } \\
\text { Dr. Patricia Kearney* } \\
\text { Claire O’Regan* }\end{array}$ \\
\hline Macular degeneration & $\begin{array}{l}\text { Professor RoseAnne Kenny* } \\
\text { Professor Stephen Beatty¥ } \\
\text { Dr. John Nolan* } \\
\text { Dr. Hilary Cronin* } \\
\text { Claire O’Regan* } \\
\text { Dr. Patricia Kearney* }\end{array}$ \\
\hline Biomarkers \& Genetics & $\begin{array}{l}\text { Professor RoseAnne Kenny* } \\
\text { Professor Michael Gill* } \\
\text { Dr. Aidan Corvin* } \\
\text { Dr. Derek Morris* } \\
\text { Dr. Hilary Cronin* } \\
\text { Claire O’Regan* } \\
\text { Dr. Patricia Kearney* } \\
\text { Professor Robert Clark§ }\end{array}$ \\
\hline
\end{tabular}

* Trinity College Dublin

† University College Cork

$\S$ University of Oxford
$¥$ Waterford Institute of Technology

₹ Royal College of Surgeons Ireland 


\section{Appendix 5: Details of Clinical Measurements Taken at the TILDA Health Centre}

\section{Summary of clinical measurements taken at health centre}

\begin{tabular}{|c|c|c|}
\hline Variables & No. of measurements & Equipment Used \\
\hline Height & One & SECA 240 wall mounted measuring rod \\
\hline Weight & One & SECA electronic floor scales or SECA seated scales \\
\hline Waist size ${ }^{a}$ & Two & Standard tape measure \\
\hline Hip size ${ }^{a}$ & Two & Standard tape measure \\
\hline Blood Pressure & Three -2 seated $\& 1$ standing & $\begin{array}{l}\text { OMRONTM digital automatic } \\
\text { blood pressure monitor }\end{array}$ \\
\hline Heart Rate & Three & $\begin{array}{l}\text { OMRONTM digital automatic } \\
\text { blood pressure monitor }\end{array}$ \\
\hline Grip Strength & Four -2 readings on each hand & Baseline Hydraulic Hand dynamometer \\
\hline Global cognition & Two & $\begin{array}{l}\text { 1. Montreal Cognitive Assessment (MOCA) } \\
\text { 2. Mini Mental State examination (MMSE) }\end{array}$ \\
\hline Depression & One & 8-item CES-D scale \\
\hline Attention & One & Sustained Attention Response Time (laptop) \\
\hline Visual Memory & One & $\begin{array}{l}\text { CAMDEX Picture Memory Test } \\
\text { (Acquisition, Free recall, Recognition) }\end{array}$ \\
\hline Speed of processing & One & Choice reaction time test (laptop) \\
\hline Executive function & Three & $\begin{array}{l}\text { Timed Colour Trails } 1 \& 2 \\
\text { Visual reasoning - CAMDEX }\end{array}$ \\
\hline Timed Up \& Go & One & Standard tape measure/Chair/tape \\
\hline Phasic Blood Pressure & $\begin{array}{l}\text { Six readings }-1 \text { at baseline, } \\
1 \text { nadir and } 4 \text { at } 30 \text { second } \\
\text { intervals after active stand }\end{array}$ & Finometer MIDI \\
\hline Pulse Wave Velocity & Two & Vicorder \\
\hline Heart Rate Variability & One 10 minute recording & Medilog Darwin AR12 \\
\hline Visual Acuity & Two - one for each eye & LOGMar chart \\
\hline Contrast Sensitivity & $\begin{array}{l}\text { Two - dim light both with } \\
\text { and without glare }\end{array}$ & Stereo Optical Co., Functional Visual Analyzer \\
\hline $\begin{array}{l}\text { Retinal Photograph } \\
\text { Macular Pigment }\end{array}$ & Two - one for each eye & NIDEX - Non-Mydriatic Auto-Fundus Camera \\
\hline Optical Density & Twelve measurements in 1 eye & Macular Metrics DensitometerTM \\
\hline Bone density & One - non dominant foot & Achilles Insight Heel Ultrasound machine \\
\hline Assessment of Gait & $\begin{array}{l}\text { Three - one normal walk, } \\
\text { one walk + cognitive task, } \\
\text { one walk + manual task }\end{array}$ & GAITRite sensored mat \\
\hline Venous Blood Sample & $25 \mathrm{mls}$ & Standard blood taking materials \\
\hline
\end{tabular}

\footnotetext{
a If the difference between two measurements is $>3 \mathrm{cms}$, a third measurement is required, with the values for the last two measurements recorded.
} 


\section{Appendix 6: Details of Clinical Measurements Taken at the TILDA Health Centre}

\section{Summary of clinical measurements taken during the home health assessment}

\begin{tabular}{|c|c|c|}
\hline Variables & No. of measurements & Equipment Used \\
\hline Height & One & SECA 240 wall mounted measuring rod \\
\hline Weight & One & SECA electronic floor scales \\
\hline Waist Size ${ }^{a}$ & Two & Standard tape measure \\
\hline Hip Size $^{a}$ & Two & Standard tape measure \\
\hline Blood Pressure & Three - 2 seated \& 1 standing & OMRONTM digital automatic blood pressure monitor \\
\hline Grip Strength & Four - 2 readings on each hand & Baseline Hydraulic Hand dynamometer \\
\hline Global Cognition & Two & $\begin{array}{l}\text { 1. Montreal Cognitive Assessment (MOCA) } \\
\text { 2. Mini Mental State examination (MMSE) }\end{array}$ \\
\hline Depression & One & 8-item CES-D scale \\
\hline Attention & One & Sustained Attention Response Time (laptop) \\
\hline Visual Memory & One & $\begin{array}{l}\text { CAMDEX Picture Memory Test } \\
\text { (Acquisition, Free recall, Recognition) }\end{array}$ \\
\hline Speed of Processing & One & Choice reaction time test (laptop) \\
\hline Executive Function & Three & $\begin{array}{l}\text { Timed Colour Trails } 1 \& \\
2 \text { Visual reasoning - CAMDEX }\end{array}$ \\
\hline Timed Up \& Go & One & Standard tape measure/Chair/tape \\
\hline Venous Blood Sample & $25 \mathrm{mls}$ & Standard blood taking materials \\
\hline
\end{tabular}

a If the difference between two measurements is $>3 \mathrm{cms}$, a third measurement

is required, with the values for the last two measurements recorded. 


\section{Appendix 7: Health Centre Assessment Feedback Form}

\section{Health Assessment}

\begin{tabular}{|c|c|c|c|}
\hline \multicolumn{4}{|l|}{ Participant Name: } \\
\hline \multicolumn{4}{|l|}{ Date of Assessment: } \\
\hline TEST & FINDING & REFERENCE RANGE & INTERPRETATION \\
\hline BLOOD PRESSURE & MMHG & $\begin{array}{l}\text { LESS THAN } 140 / 90 \text { MMHG } \\
\text { LESS THAN } 135 / 85 \text { IN PEOPLE WITH DIABETES }\end{array}$ & \\
\hline WEIGHT & kg (st lbs) & & \\
\hline HEIGHT & $\mathrm{cm}(\mathrm{ft} ")$ & & \\
\hline HEEL BONE ULTRASOUND & T Score & $\begin{array}{l}+2 \text { to }-1=\text { NORMAL } \\
-1 \text { to }-2.5=\text { REDUCED } \\
-2.5 \text { and below = MAY INDICATE } \\
\text { OSTEOPOROSIS BUT NEEDS } \\
\text { FURTHER ASSESSMENT }\end{array}$ & \\
\hline $\begin{array}{l}\text { VISION } \\
\text { LOGMAR }\end{array}$ & $\begin{array}{l}\text { Right Eye: } \\
\text { Left Eye: }\end{array}$ & $\begin{array}{l}0.2 \text { to }-0.3=\text { NORMAL } \\
1.0 \text { to } 0.2=\text { REDUCED }\end{array}$ & $\begin{array}{l}\text { Right Eye: } \\
\text { Left Eye }\end{array}$ \\
\hline
\end{tabular}

\section{NOTES}

The interpretation of findings is based on information from previous studies linking these factors with health risk. Participants with undesirable findings may be at higher risk of some diseases, and may wish to consider appropriate lifestyle changes (e.g. stopping smoking; reducing dietary intake of salt, meat and other sources of animal and other "saturated" fats; increasing physical activity). Some advice leaflets are available from reception. Participants with high blood pressure levels may also wish to have their blood pressure checked again by their own doctor. Please note that local GPs are not part of the TILDA study therefore the normal charge for a consultation with your doctor will apply. 


\section{Appendix 8: Home Assessment Feedback Form}

\section{Health Assessment}

\begin{tabular}{|c|c|c|c|}
\hline \multicolumn{4}{|l|}{ Participant Name: } \\
\hline \multicolumn{4}{|l|}{ Date of Assessment: } \\
\hline TEST & FINDING & REFERENCE RANGE & INTERPRETATION \\
\hline \multirow[t]{2}{*}{ BLOOD PRESSURE } & $\longrightarrow$ & LESS THAN 140/90 MMHG & \\
\hline & MMHG & LESS THAN 135/85 IN PEOPLE WITH DIABETES & \\
\hline WEIGHT & \multicolumn{3}{|l|}{ _kg } \\
\hline HEIGHT & \multicolumn{3}{|l|}{$\ldots \mathrm{cm}$} \\
\hline BLOOD RESULTS WIL & V BY POST & & \\
\hline
\end{tabular}

\section{NOTES}

Participants with high blood pressure may be at a higher risk for heart disease and may wish to consider appropriate lifestyle changes (e.g. stopping smoking; reducing dietary intake of salt, meat and other sources of animal and other "saturated" fats; increasing physical activity). Participants with high blood pressure levels may also wish to have their blood pressure checked again by their own doctor. Please note that local GPs are not part of the TILDA study therefore the normal charge for a consultation with your doctor will apply. 


\section{Appendix 9: Blood Results Feedback Form (for both centre and home health assessments)}

Dear Participant,

Many thanks for participating in the TILDA Health Assessment.

We have now received the results of your blood tests and the results are detailed below.

\begin{tabular}{|l|l|l|l|}
\hline TEST & FINDING & REFERENCE RANGE & INTERPRETATION \\
\hline CHOLESTEROL TOTAL & $\mathrm{mmol} / \mathrm{L}$ & Less than 5.0 Normal & \\
\hline TRIGLYCERIDES & $\mathrm{mmol} / \mathrm{L}$ & Between $0.60-2.0$ Normal & \\
\hline DIRECT LDL & $\mathrm{mmol} / \mathrm{L}$ & Less than 3.0 Normal & \\
\hline $\mathrm{HDL}$ & $\mathrm{mmol} / \mathrm{L}$ & Between $1.00-1.90$ Normal & \\
\hline
\end{tabular}

The interpretation of findings is based on information from previous studies linking these factors with health risk. Participants with undesirable findings may be at higher risk of some diseases, and may wish to consider appropriate lifestyle changes (e.g. stopping smoking; reducing dietary intake of salt, meat and other sources of animal and other "saturated" fats; increasing physical activity). If you have given consent, a copy of these results will be sent to your doctor shortly. Please note that local GPs are not part of the TILDA study therefore the normal charge for a consultation with your doctor to discuss these results will apply.

If you have any questions regarding the above results, please do not hesitate to contact the Tilda Nurses on 018962509.

Yours sincerely,

\section{P. Walsh}

\section{Executive Officer}

The Irish Longitudinal Study on Ageing 


\section{Appendix 10: Cholesterol Information Sheet}

\section{Cholesterol}

\section{Why is cholesterol important?}

Having high cholesterol levels in your blood is one of the risk factors which increases your chances of getting heart disease.

\section{What is cholesterol?}

Cholesterol is a type of fat found in your blood. You need a certain amount of cholesterol for all your body cells and to produce important hormones. However, if there is too much cholesterol in your blood, it sticks to the inner lining of your artery or blood vessels to form atheroma. As a result of this build-up on the artery walls, your arteries become narrowed.

This process is called hardening of the arteries or atherosclerosis.

- If an artery supplying the heart muscle becomes blocked completely, the heart muscle becomes damaged. This is known as a heart attack.

- If an artery to the brain is completely blocked, it damages the brain. This is called a stroke.

\section{Where does cholesterol come from?}

Cholesterol is made in the body mainly by the liver. This is often called blood cholesterol. The body can produce all the cholesterol it needs to carry out its many functions and can usually maintain a healthy level of blood cholesterol. However, sometimes the balance goes wrong and there is an increase in blood cholesterol. This may result from inherited problems or from eating too much saturated (animal) fat or too many foods from the top shelf of the Food Pyramid (see pages 8 and 9).

Some foods such as eggs, liver and shellfish contain cholesterol. However, the amount of cholesterol in these foods does not greatly affect the amount of cholesterol in your blood. You can eat foods that contain cholesterol in moderation as part of a healthy varied diet.

\section{There are three main types of cholesterol -}

HDL

HDL (high density lipoprotein) cholesterol is called good cholesterol or healthy cholesterol, because it mops up cholesterol left behind in your arteries and carries it to the liver where it is broken down and passed out of the body. Regular physical activity and exercise can help increase your HDL level. High levels of HDL cholesterol can protect you against getting heart disease.

LDL

LDL (low density lipoprotein) cholesterol travels from the liver through the arteries to other parts of the body. LDL is called bad cholesterol because it sticks to the walls in your arteries - making them narrow. This reduces the blood supply to your heart or brain. Eating too many foods high in saturated fat can raise your LDL cholesterol. High levels of LDL cholesterol increase your risk of heart disease. 


\section{Triglycerides}

Triglycerides are another type of fat found in your blood. Too much triglyceride in your blood can increase your chances of getting heart disease.

\section{Is your cholesterol level too high?}

If your results show a blood cholesterol level greater than five $\mathrm{mmol} / \mathrm{l}$, ask your doctor to arrange for another test. You will need to fast for 12 hours to get more information on your HDL and LDL cholesterol. If you already have had a heart attack, bypass surgery or angioplasty, it is very important that you keep your LDL below $2.6 \mathrm{mmol} / \mathrm{l}$. Your doctor will most likely suggest medication together with healthy eating and other lifestyle changes outlined in this booklet.

As well as HDL and LDL levels, the more detailed test will show triglyceride levels - another type of fat. If you need to change any aspects of your cholesterol or triglyceride levels, your doctor can advise you on changes in lifestyle and may recommend medication. If you are prescribed medication, it is important that you follow the instructions carefully.

\section{How can I lower my cholesterol?}

- Get down to a healthy weight - being overweight means your heart has to work harder to pump blood around the body.

- $\quad$ Eat oily fish twice a week.

- $\quad$ Eat more fruit and vegetables.

- $\quad$ Eat more wholegrain cereals and breads, plenty of jacket potatoes, rice and pasta.

- Choose lean meats. Trim fat off meat and skin off chicken.

- Drain oil from cooked dishes containing minced meat.

- Choose low-fat dairy products.

- Choose low-fat spreads made from sunflower or olive oil.

- Choose less foods from the top shelf of the Food Pyramid.

- Use low-fat healthy ways of cooking, like grilling or oven-baking, instead of frying. 


\section{What can I do if my triglycerides are too high?}

- $\quad$ Follow the general advice above for lowering your blood cholesterol - this will also help lower your triglycerides.

- $\quad$ Oily fish, such as sardines, mackerel, trout or salmon can help lower your triglyceride levels. Try to eat oily fish (fresh or tinned) twice a week, for example, one main meal and one lunch serving. Oily fish, which contains n-3 or omega 3 fatty acids, improves the blood circulation, reduces the stickiness of the blood and prevents your blood from clotting.

- If you drink alcohol, take no more than one drink (such as a small glass of wine or a half pint of beer) a day and go some days without any alcohol.

- Watch the amount of sweet foods you eat from the top shelf of the Food Pyramid, like biscuits, cakes, chocolate, sweets, fizzy drinks, jams, marmalades and sugars.

- Choose low-sugar or sugar-free fizzy drinks, mixers and tinned fruits in their own juice. Instead of sugar try artificial sweeteners.

\section{What if I have low HDL?}

If your blood test showed that your HDL cholesterol is below the recommended levels, you will need to increase this level to reduce your risk of heart disease.

\section{You can increase the healthy HDL levels by:}

- being more active;

- $\quad$ reducing your weight; and

- if you smoke, by stopping smoking.

- Your doctor can also prescribe medication to raise your HDL levels. 
tilda

The Irish Longitudinal

Study on Ageing
The Irish Longitudinal Study on Ageing

Chemistry Extension Building Trinity College Dublin

Dublin 2

Republic of Ireland

Tel: +353 18964120

Email: tilda@tcd.ie

Website: www.tilda.ie 